\title{
Transport of lipid vesicles via the cilia logistic network in the brain of mice
}

\author{
DISSERTATION \\ for the award of the degree \\ "Doctor rerum naturalium" \\ of the Georg-August-Universität Göttingen \\ within the doctoral programm \\ Physics of Biological and Complex Systems \\ of the Göttingen Graduate School of Neurosciences, Biophysics, and \\ Molecular Biosciences (GGNB) \\ of the Georg-August University School of Science (GAUSS)
}

submitted by

Ann-Kathrin Günther

from Hildesheim

Göttingen, 2018 


\section{Betreuungsausschuss}

Prof. Dr. Gregor Eichele, Max-Planck Institut für biophysikalische Chemie

Prof. Dr. Eberhart Bodenschatz, Max-Planck Institut für Dynamik und Selbstorganisation

Prof. Dr. Christoph F. Schmidt, III. Physikalisches Institut der Georg-August-Universität Göttingen

Mitglieder der Prüfungskommission

Referent: $\quad$ Prof. Dr. Gregor Eichele,

Max-Planck Institut für biophysikalische Chemie

Korreferent: $\quad$ Prof. Dr. Eberhart Bodenschatz,

Max-Planck Institut für Dynamik und Selbstorganisation

Weitere Mitglieder der Prüfungskommission

Prof. Dr. Jens Frahm, Max-Planck Institut für biophysikalische Chemie

Dr. Karen Alim, Max-Planck Institut für Dynamik und Selbstorganisation

Dr. Thomas P. Burg, Max-Planck Institut für biophysikalische Chemie

Tag der mündlichen Prüfung: 21.09.2018 
The world is made up of four elements: Earth, Air, Fire and Water. That is a fact well known even to Corporal Nobbs. It's also wrong. There's a fifth element, and generally it's called Surprise - The Truth

Terry Pratchett 


\section{Abstract}

The ventricular system of the mammalian brain consists of four interconnected cavities, which are lined by ciliated ependymal cells [1]. Constant and coordinated movement of the cilia results in a directed flow of cerebrospinal fluid (CSF) along the ependymal walls. In the ventral part of the third ventricle $(\mathrm{v} 3 \mathrm{~V})$ the cilia give rise to a complex network of flow that confines and connects regions and changes throughout the day. This so-called ciliary logistic network (CLN) [2] may provide transport routes for CSF components and has the potential to guide and restrict the distribution of local secretions.

The choroid plexus is located in each of the four ventricles and constantly secretes CSF and its constituents [3]. One of these constituents are extracellular vesicles (EVs), namely exosomes. These exosomes vary in size between $30-150 \mathrm{~nm}$ [4], are transported within the ventricular system and exert physiologic functions in the central nervous system [5]. Exosomes consist of a lipid bilayer that encloses the content and this way serves as cargo shuttle. At the secretory tissue, exosomes are filled with different components [6] that subsequently may be targeted to and absorbed by specific regions [3] presumably involving interaction of membrane-bound protein ligands and protein receptors on both, the surface of the exosome and the target region, respectively.

It is unclear, how exosomes are distributed throughout the ventricular system and whether the CLN mediates site-specific deliveries of exosomes. In this thesis, a characterization of exosome-like cargo transport along the CLN and potential target sites in the $\mathrm{v} 3 \mathrm{~V}$ are presented. To investigate the transport and interaction of exosome-like cargo, fluorescently-labelled liposomes of defined composition and size were applied to the ciliagenerated near wall flow via microinjections.

Small liposomes droplets were applied in each flow region of the v3V. The transport direction of liposomes in each region matched the previously described flow pattern [2]. Further, liposomes allowed the visualization of fine cilia-generated streamlines within the near wall flow along the ventricular wall. Liposomes applied at the head of the inflow region were distributed over a large area in fine separated lines, while liposomes released in other regions were transported along a single line. These findings demonstrate the precision of the CLN and reveal the underlying fine structure of cilia-generated flow.

In addition, liposomes were shown to attach to ependymal cell cilia while transported along the cilia-generated flow. Homogeneous exposure of the $\mathrm{v} 3 \mathrm{~V}$ to diluted liposome solution revealed a higher affinity of liposome attachment in specific regions of the $\mathrm{v} 3 \mathrm{~V}$. 
Moreover, liposome attachment was shown to depend on the lipid composition used for liposome preparation. These results indicate, that cargo and target site specific interaction is needed in addition to site specific delivery of the cargo by the CLN.

Following these results, exosomes secreted from Z310 rat choroid plexus cells were isolated from cell culture supernatant, characterized, fluorescently-labelled and applied to the cilia-generated near wall flow of $\mathrm{v} 3 \mathrm{~V}$. The results suggest, that exosomes are transported along the CLN similarly to liposomes. Further experiments are needed to clarify the transport and interaction of exosomes within the CLN in detail. 


\section{Contents}

List of Figures $\quad$ xi

Symbols and Abbreviations xiii

1 Introduction 1

1.1 The ventricular system of the brain . . . . . . . . . . . . . 2

1.1.1 Cilia-generated near wall flow in the ventral third ventricle . . . . 3

1.1.2 The epithilium of the ventricular system and cilia . . . . . . . 3

1.2 Extracellular vesicles . . . . . . . . . . . . . . . . 6

1.3 Introduction to lipids and liposomes . . . . . . . . . . . . . . 10

1.3.1 Formation and classification of liposomes . . . . . . . . . . 11

2 Materials and Methods $\quad 13$

2.1 Collaborations . . . . . . . . . . . . . . . . . . . 13

2.2 Animals .............................. 13

2.2.1 Animal housing and breeding . . . . . . . . . . . 13

2.2.2 Circadian entrainment of animals . . . . . . . . . . . . 14

2.3 Vesicle preparation for cilia logistic network characterization . . . . . . . 14

2.3.1 Chemicals and Reagents . . . . . . . . . . . . . . . . . . . . 14

2.3.2 Preparation of fluorescently-labeled liposomes . . . . . . . . 15

2.3.3 Compositions of fluorescently-labeled liposomes . . . . . . . . 17

2.3.4 Measurement of liposome concentration and size distribution . . . 19

2.3.5 Preparation of extracellular vesicles . . . . . . . . . . . . . . . 19

2.4 Analysis of cilia-generated flow in the ventral third ventricle . . . . . . 23

2.4.1 Preparation of the ventral third ventricle: open pouch . . . . . . 23

2.4.2 Imaging cilia-generated flow in the ventral third ventricle . . . . . 24

2.4.3 Flow maps: Analysis of cilia-generated flow . . . . . . . . . 25 
2.5 Characterization of liposome distribution in the ventral third ventricle . . 26

2.5.1 Injection of fluorescent liposomes into cilia-generated near wall flow ........................ 26

2.5.2 Analysis of fluorescent liposome propagation in near wall flow . . 27

2.6 Interaction of liposomes with ventral third ventricle ependymal cells . . . 27

2.6.1 Applying liposome to open pouch preparation . . . . . . . . . . 28

2.6.2 Applying liposome to side view preparation . . . . . . . . . . 28

2.6.3 Incubation of ventral third ventricle explant in liposome solution . 28

2.6.4 Live imaging of liposome-labeled cilia . . . . . . . . . . . 31

2.6.5 Staining of ventral third ventricle . . . . . . . . . . 31

2.7 Interaction of extracellular vesicles with ventral third ventricle ependymal cells ............................. 32

2.7.1 Incubation of ventral third ventricle in extracellular vesicle solution 32

2.7.2 Applying extracellular vesicle to side view preparation . . . . . 32

2.8 Liposome interaction with diverse cell types . . . . . . . . . . . 33

2.8.1 Mouse trachea tissue and cells . . . . . . . . . . . . . 33

2.8 .2 Mouse oviduct . . . . . . . . . . . . . . . . . . . 34

2.8 .3 Rabbit oviduct . . . . . . . . . . . . . . . 34

2.8.4 Mouse sperm cells . . . . . . . . . . . . . . . . . . . . . . . . . . . . . . . . . . . .

2.8.5 Human nasal epithelia cells . . . . . . . . . . . . 35

2.9 Experimental workflow . . . . . . . . . . . . . . . . 35

3 Results $\quad 37$

3.1 Characterization of liposome transport via the cilia logistic network . . . 37

3.1.1 Analysis of liposome properties . . . . . . . . . . . . 37

3.1.2 Propagation of liposomes in cilia-generated near wall flow . . . . 40

3.1.3 Analysis of liposome propagation rates . . . . . . . . . . . 43

3.1.4 Transport of liposomes via the cilia network at Zeitgeber times $10 \mathrm{~h}$ and $23.5 \mathrm{~h} \ldots \ldots \ldots \ldots \ldots$. . . . . . . . . . . . . . . . . . . . . .

3.2 Liposome interaction with ependymal cilia . . . . . . . . . . . . . . 46

3.2.1 Liposome attachment to ependymal cells and cilia in the ventral third ventricle . . . . . . . . . . . . . . . . . 47

3.2.2 Liposomes-cilia interaction in dependence of liposome lipid-composition ......................... 59 
3.2.3 Monitoring liposome-ependymal cilia interactions . . . . . . . 65

3.2.4 Localization and analysis of liposome-ependymal cilia attachment mechanism . . . . . . . . . . . . . 6 65

3.2.5 Concentration and time dependence of liposome attachment . . . 72

3.2.6 Interaction of liposomes with cilia and flagella . . . . . . . . 77

3.3 Interaction of EVs produced by Z310 cells with ependymal cilia and flagella 80

3.3.1 Characterization of EVs isolated from Z310 rat choroid plexus cells 80

3.3.2 Transport and interaction of Z310 EVs with v3V ependymal cells 81

3.3.3 Interaction of Z310 EVs with mouse sperm flagella . . . . . . . 84

3.3.4 Specificity of EV binding . . . . . . . . . . . . 84

4 Discussion

89

4.1 Transport of extracellular vesicle like cargo in cilia-generated flow of the $\mathrm{v} 3 \mathrm{~V} \ldots \ldots \ldots \ldots \ldots$

4.2 The interaction of extracellular vesicle-like CSF components with ependymal cells of the $\mathrm{v} 3 \mathrm{~V} \ldots \ldots \ldots$. . . . . . . . . . . . . . . 91

5 Appendix $\quad 93$

5.1 Calculating the mean displacement of small particles caused by diffusion 93

5.2 List of experiments and mice . . . . . . . . . . . . . . 95

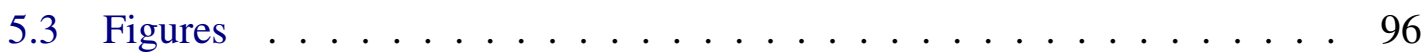

$\begin{array}{ll}\text { Bibliography } & 101\end{array}$

$\begin{array}{ll}\text { Acknowledgement } & 113\end{array}$ 
Contents 


\section{List of Figures}

1.1 Ventricular system of a mouse brain . . . . . . . . . . . . . . 4

1.2 Biogenesis of extracellular vesicles . . . . . . . . . . . 8

1.3 Phospholipids and lipid bilayers . . . . . . . . . . . . . . . 12

2.1 Experimental schema of imaging cilia-generated flow in v3V . . . . . . 24

2.2 Experimental schema of $\mathrm{v} 3 \mathrm{~V}$ incubation in liposome solution . . . . . . . 29

2.3 Experimental workflow for liposomes . . . . . . . . . . . . 36

2.4 Experimental workflow for extracellular vesicles $\ldots \ldots . \ldots 36$

3.1 Characterization of liposomes . . . . . . . . . . . . . . . 39

3.2 Propagation of liposomes in ciliary near wall flow: right hemisphere . . . 41

3.3 Propagation of liposomes in ciliary near wall flow: left hemisphere . . . . 42

3.4 Velocity distribution of liposomes in cilia near wall flow . . . . . . . . . 44

3.5 Propagation of liposomes in ciliary near wall flow . . . . . . . . . . . . . 46

3.6 Attachment of liposomes to ependymal cells . . . . . . . . . . . . . . . . 47

3.7 Localization of liposome attachment in $\mathrm{v} 3 \mathrm{~V}$. . . . . . . . . . . . . . . . 49

3.8 Map of liposome atttachment site in v3V after incubation: Composition II 50

3.9 Flow map of v3V explants before and after liposome incubation . . . . . 52

3.10 Velocity distribution of cilia-generated flow in the v3V over the cause of incubation experiments . . . . . . . . . . . . . . 54 54

3.11 Cilia beating frequency distribution before and after adding liposomes . . 55

3.12 Localisation of liposome attachment in cilia beating bundles of flow 6 and 757

3.13 Cilia beating frequency in flow 6 and flow $7 \ldots \ldots$. . . . . . 58

3.14 Liposome-cilia interaction in dependence of the liposome lipid composition prepared with size exclusion chromatography . . . . . . . . . 63

3.15 Liposome-cilia interaction in dependence of the liposome lipid composition prepared with extrusion $\ldots \ldots$. . . . . . . . . . . 6 64 
3.16 Transport of liposomes along v3V preparation . . . . . . . . . . . . 69

3.17 Localisation of liposome attachment in flow 6 and 7 . . . . . . . . . 70

3.18 Comparison of liposome attachment after incubation and injection in v3V 71

3.19 Kinetic of liposome attachment to v3V ependymal cilia and cells: Inflow . 74

3.20 Kinetic of liposome attachment to v3V ependymal cilia and cells: Separatrix 75

3.21 Kinetic of liposome attachment to v3V ependymal cilia and cells: Distributor ......................... 76

3.22 Attachemnt of liposomes to cilia and flagella . . . . . . . . . . . . 79

3.23 Characterization of EVs isolated from Z310 rat choroid plexus cells . . . 82

3.24 Transport of Z310 cell EVs along v3V side view preparation . . . . . . 83

3.25 Flow maps of v3V before and after incubation with Z310 cell EVs . . . 86

3.26 Interaction of Z310 cell EVs with sperm flagella . . . . . . . . . . . 87

5.1 Schema of coordinates in regard to the ependymal wall . . . . . . . . . 94

5.2 Wound closure assay . . . . . . . . . . . . . . . . . . . 96

5.3 Map of liposome atttachment site in v3V after incubation: Composition III 97

5.4 Map of liposome attachment site in v3V after incubation: Composition IV 98

5.5 Map of liposome atttachment site in v3V after incubation: Composition VIII . . . . . . . . . . . . . . . . . . . . . . . . . . 99

5.6 Map of liposome atttachment site in v3V after incubation: Composition IX 100

5.7 Liposome attachment cilia of to Cdkl5 mouse mutante . . . . . . . . . . 100 


\section{Symbols and Abbreviations}

\begin{tabular}{lll} 
Symbol & Meaning & Unit \\
\hline $\mathrm{n}$ & Number of animals, samples or experiments & pcs
\end{tabular}

\begin{tabular}{ll} 
Abbreviation & Meaning \\
\hline $3 \mathrm{~V}$ & third ventricle \\
$\mathrm{ac}$ & anterior commissure \\
Alix & programmed cell death 6 interacting protein (PDCD6IP) alias \\
& ALG-2-interaction protein X \\
AGO2 & argonaute-2 \\
ChP & choroid plexus \\
CM & conditioned media \\
CSF & cerebral spinal fluid \\
d3V & dorsal third ventricle \\
DMEM & Dulbecco's modified eagle medium \\
DOPC & 1,2 -dioleoyl-sn-glycero-3-phosphocholine \\
DOPE & 1,2 -dioleoyl-sn-glycero-3-phosphoethanolamine \\
DOPS & 1,2 -dioleoyl-sn-glycero-3-phospho-L-serine \\
EM & electron microscopy \\
ESCRT & endosomal sorting complex required for transport \\
EVs & extracellular vesicles \\
Flot 2 & Flotillin-2 \\
ILVs & intraluminal bodies \\
lncRNA & long non-coding ribonucleic acid \\
mCLING & membrane-binding fluorophore-cysteine-lysine-palmitoyl group \\
microRNA & micro ribonucleic acid \\
mRNA & messenger ribonucleic acid \\
MVBs & multivesicular bodies \\
&
\end{tabular}


NTA nano particle tracking analysis

PBS phosphate-buffered saline

PA phosphatidic acid

PC phosphatidylcholine

PE phosphatidylethanolamine

PEG Precipitation by Polyethylene glycol

PG phosphatidylglycerol

PI phosphatidylinositol

PS phosphatidylserine

ROI region of interest

RNA ribonucleic acid

RT room temperature

SECH size exclusion chromatography

siRNA small interfering ribonucleic acid

SM sphingomyelins

SUV small unilamellar vesicles

$\mathrm{tz} \quad$ tanycyte region

UC ultracentrifugation

v3V ventral third ventricle

VPS4 Vacuolar protein sorting-associated protein 4

ZT zeitgeber time 


\section{Chapter 1}

\section{Introduction}

The cerebral spinal fluid (CSF) contains many biologically relevant substances, for example signalling molecules, nutrients and extracellular vesicles (EVs) [3, 7]. The latter have been shown to induce physiological functions [5] and are secreted by diverse cell types of the brain parenchyma and the choroid plexus [3]. However, it is still unknown how extracellular vesicles are transported from their production site to a given target site across the ventricular lumen.

Cilia-generated near wall flow enables the precise delivery of CSF components along the ependymal wall of the ventricular system of the brain. Secreted by any local source close to or in the ventricular system, EVs may be transported by the cilia-generated flow patterns.

The cilia-generated flow analysis technique for the ventral third ventricle [2], which was previously developed in the lab, was used to address the question of EVs transport along the ependymal walls and possible interaction of EVs with particular regions. The EV content and protein composition on their surface were shown to differ significantly depending on their source and the state of the tissue of origin [3, 8]. This diversity suggests a high level of specificity, which has to be taken into consideration in terms of EVs interaction with potential targets. To simplify the investigation of EVs distribution and tissue interaction, small unilamellar vesicles were used instead.

Small unilamellar vesicles, which are one class of so-called liposomes, were produced in vitro with controlled composition, content and size $[9,10]$. Henceforth the term liposomes will be used only. Fluorescently-labeled liposomes were then injected into ciliagenerated near wall flow of the ventral third ventricle (v3V) to characterize the distribution of EV-like cargo. Furthermore, fluorescent liposomes were used to map potential target 
sites. Then, the interaction of fluorescent liposomes and fluorescently-labeled EVs, isolated from the Z310 choroid plexus cell line, with v3V ciliated epithelia cells was studied.

\subsection{The ventricular system of the brain}

The ventricular system of the adult brain consists of four interconnected cavities, two lateral ventricles, the third ventricle and the fourth ventricle, which are embedded in the brain parenchyma and filled with cerebrospinal fluid (CSF) (Fig. 1.1 A). The two lateral ventricles, each located in one hemisphere, are connected to the third ventricle (3V) through the interventricular foramina (Monro). The third ventricle connects with the fourth ventricle via the cerebral aqueduct (Sylvius). The fourth ventricle is connected to the central canal of the spinal cord and to the subarachnoid cisterns, which are connected via the median aperture and the right and left lateral apertures [7, 11, 12].

The walls of the ventricular system are lined with ependymal cells [1, 13]. Ependymal cells are cuboidal to columnar in shape, covered with microvilli on their apical surface and motile cilia (Fig. 1.1 B) [1,13]. Motile cilia are organized in bundles at one side of the cell and exert coordinated beating that gives rise to directional flow of CSF [14, 15]. Microvilli are thin extensions emerging from the plasma membrane, containing crosslinked actin filaments and thereby enlarge the cell surface [16]. Exposed to the lumen above the ependymal cells, microvilli increase the absorption and secretion ability of the cell $[1,16]$. Neighbouring ependymal cells join by zonula adherens type junctions close to the apical surface and gap junctions near the basal surface of the cells [1].

Specialized secretory ependymal cells linked by tight junctions form the epithelium of the choroid plexus (ChP), which protrudes into each ventricle [12]. The choroid plexus tissue is innervated with blood vessels and secretes cerebrospinal fluid (CSF) into the ventricular cavities $[1,17]$. Ions, nutrients, signalling molecules and extracellular vesicles are secreted into the ventricular system via the $\mathrm{ChP}[7,17]$. The choroid plexus forms the blood-CSF-barrier and maintains the brain homeostasis [1, 17]. The largest amount of CSF is produced by the lateral choroid plexi giving rise to a CSF bulk flow from the lateral ventricles through the third ventricle towards the fourth ventricle [12]. In the fourth ventricle, CSF leaves the ventricular system and circulates around the brain parenchyma until reabsorbed to blood or lymph via various pathways [7, 12]. Vascular pulsation during the cardiac cycle was observed to causes an oscillatory CSF movement in the ventricular lumen in addition to respiratory cycle driven CSF flow [7, 18-21]. However, CSF flow 
along and close to the ventricular walls of the ventricular system is dominated by ependymal cilia generated flow $[2,19]$. Components released into the CSF are transported by bulk flow and cilia-generated near wall flow although the transport pathways and target sites are largely unknown.

\subsubsection{Cilia-generated near wall flow in the ventral third ventricle}

The third ventricle is a narrow cavity located along the midline of the brain. Figure $1.1 \mathrm{~A}$ shows the position of the third ventricle (blue) within the adult mouse brain. The ventral part is positioned below the thalamus and faced by multiple neurosecretory hypothalamic nuclei [2]. CSF enters the ventral third ventricle (v3V) through the anterior duct and leaves the $\mathrm{v} 3 \mathrm{~V}$ through the posterior duct. The cilia-generated near wall flow along the ventricular walls of the ventral third ventricle (v3V) was shown to form a complex flow pattern, which forms flow domains and thereby connects and seperates areas of the v3V (Fig. 1.1) [2]. After passing the anterior commissure at the entry of the v3V the ciliagenerated flow fans out into flow 1, flow 2 and flow 3 [2]. The first flow is directed anteriorly towards the optical recess. The second is directed ventrally towards the base of the ventricle, while the third flows posteriorly (Fig. 1.1 C). Flow 4, which starts at the so-called distributor and is directed anteriorly, opposes flow 3. Thereby flow 3 and flow 4 form the separatrix, an intraventricular flow boundary. Ventrally of the separatrix flow 3 flows into flow 5, which is directed towards the base of the v3V. Flow 6 starts at the distributor and flows ventrally towards the base of the ventricle, close to the tanycyte region (tz). Tanycytes are in contact with CSF and have basal processes that connect with blood vessels and may be involved in humoral regulation [1,24]. Cells within this region are without motile cilia bundles and thus do not actively produce flow $[1,2]$. Above the tanycyte region flow 7 flows dorsally towards the posterior duct.

\subsubsection{The epithilium of the ventricular system and cilia}

The most of the ependymal wall consists of multiciliated ependymal cells. They derive from radial glial cells [25] and actively propel CSF along the ventricular walls by constant cilia beating. The ependymal lining shields the underlying parenchyma but also allows the passage of substances between the parenchyma and CSF [26].

Beside ependymal cells other cell types are present in the ventricular wall as well. Dendritic processes of neurons extent between ependymal cell bringing them into direct 
A
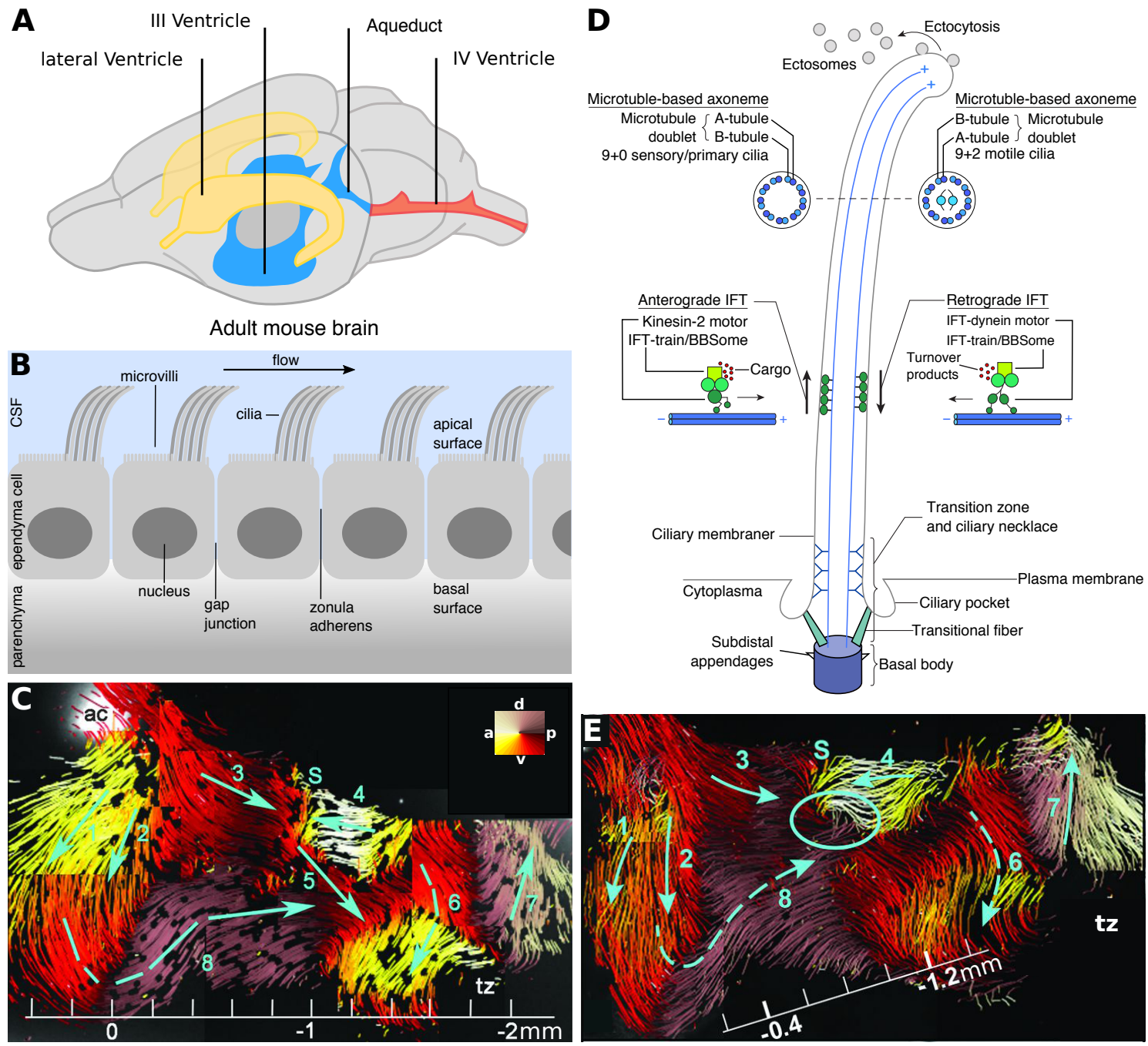

Figure 1.1: The anatomy of the ventricular system of the adult mouse brain and flow map of the v3V. A The ventricular system consists of four interconnected cavities filled with CSF produced by the choroid plexus in all four ventricles. The two lateral ventricles (yellow) are connected with the third ventricle (blue) via the interventricular foramina. The dorsal and ventral part of the third ventricle reunite at the aqueduct, which leads to the fourth ventricle (red). The image of the adult mouse brain was altered after a figure from [22]. B Scheme of multiciliated ependymal cells that line the ventricular system and generate directional flow. C Flow map of the ciliary near wall flow in the v3V at ZT10 [2]. The colour code legend indicates the flow direction in $\mathbf{C}$ and $\mathbf{E}$. The scale bars in $\mathbf{C}$ and $\mathbf{E}$ show the bregma level. Light turquoise arrows and numbers mark the main flow regions. $a c=$ anterior commissure, $\mathrm{a}=$ anterior, $\mathrm{d}=$ dorsal, $\mathrm{p}=$ posterior, $\mathrm{v}=$ ventral, $\mathrm{S}=$ separatrix and $\mathrm{tz}=$ tanycyte region. $\mathbf{D}$ Schema of primary and motile cilia axoneme. The scheme is modified from [23]. E Flow map of the ciliary near wall flow in the v3V at ZT23.5 [2]. 
contact with the CSF [1]. Moreover, supraependymal axons form a network on top of the ependymal cell layer [1].

In the lateral ventricle it was shown, that the apical endings of neural stem cells are in direct contact with the CSF [27]. The neural stem cell endings are surrounded by cells, which form a pinwheel like structure around them [27] and are embedded in the ependymal cell layer. Additionally, cells with only two cilia were found [27].

Hypothalamic tanycytes are found along the ventro-lateral ventricular wall and floor of the $\mathrm{v} 3 \mathrm{~V}$ and are involved in trophic and metabolic processes [28, 29]. They have access to CSF within the ventricle and long processes, that reach into the surrounding hypothalamic nuclei $[28,29]$. These processes are in contact with capillaries or end close to neurons $[28,29]$. Two subpopulations, $\alpha$ and $\beta$ tanycytes are distinguishable according to their position within the $\mathrm{v} 3 \mathrm{~V}$ and the location of their processes [28, 29]. $\beta$ tanycytes are divided into $\beta_{2}$ tanycytes, which occupy the floor of the $\mathrm{v} 3 \mathrm{~V}$ and extent their processes into the median eminence, and $\beta_{1}$ tanycytes, which are located more dorsal and have processes that reach into the arcuate nucleus [28, 29]. $\alpha$ tanycytes are found dorsally of the $\beta$ tanycytes and are divided into $\alpha_{1}$ and $\alpha_{2}$ subpopulations. $\alpha_{1}$ tanycytes are located dorsal of the $\alpha_{2}$ tanycytes. The processes of $\alpha$ tanycytes extend into the ventromedial and dorsomedial nucleus $[28,29]$. All tanycytes have primary cilia but lack beating cilia bundles.

\section{Structure and function of cilia}

In general, eukaryotic cilia are thin hair-like structures, which project from the cell body and perform various functions: 1) cell motility, 2) sensory reception, 3) signalling-depending control of the intracellular physiology, 4) embryonic development, 5) intercellular communication through extracellular vesicles (Fig. 1.1 D) [23, 30-35]. The cilium is a protrusion of the cell consisting of an axoneme covered with a specialized membrane [23]. In general, two types of cilia are distinguished from each other: primary cilia and motile cilia. The axoneme of primary cilia is based in a ring of nine microtubule doublets (9+0 axoneme) and motile cilia are characterized by an additional central pair of microtubules (9+2 axoneme) [23]. The microtubule doublets are made from $\alpha \beta$-tubulin subunits with the plus end pointing towards the ciliary tip. A vesicular transport network in the cytoplasm mediated delivery and removal of ciliary components to the base of the cilium [23]. These components are transported along the axoneme by the intraflagellar 
transport systems. Anterograde transport involves kinesin-2 motors and delivers cargo to the cilia tip, whereas retrograte transport regulates the removal of components from the cilia tip [23]. Components can also leave the cilium by budding of extracellular vesicles from the ciliary membrane $[23,35,36]$.

\section{Ependymal cell cilia and the third ventricle}

Ependymal cells in the ventricular system of the brain have cilia apically facing the ventricular lumen. Multiciliated ependymal cells (E1 cells) have around 50 cilia [37-39], organized in a bundle at one edge of the cell. These cilia bundles beat in a coordinated way and thereby generate a directed CSF flow near the ependymal wall [13-15]. Along the walls of each ventricle E1 cells mix with a rare subpopulation of biciliated ependymal cells (E2 cells) [37]. Both, E1 and E2 cell cilia show the 9+2 axoneme structure of motile cilia and have a length of $10-14 \mu \mathrm{m}$ and a diameter of $200 \mathrm{~nm}$ [37]. The ciliary surface is covered with receptor proteins [40].

In the posterior region of the $\mathrm{v} 3 \mathrm{~V}$ and the posterior duct of the $3 \mathrm{~V} E 2$ cells form an almost uniform band along the ventral midline [37]. Ventral of the E2 cell band in the $\mathrm{v} 3 \mathrm{~V}$ an area of almost uniform uniciliated cells (E3 cells) forms along the ventricular floor close to the ventromedial hypothalamus and the median eminence [37]. A much smaller population of E3 cells is also found in the preoptic area of the v3V [37]. These E3 cells have a single, approximately $5 \mu \mathrm{m}$ long cilium with the 9+0 axoneme structure of primary cilia [37]. Based on their structure and localisation E2 and E3 cells in the v3V might correspond to subtypes of tanycyte cells [37].

\subsection{Extracellular vesicles}

Cells of different organisms, eukaryotic and also prokaryotic, release membrane vesicles into the extracellular environment $[6,41,42]$. These extracellular vesicles (EVs) consist of a lipid bilayer that encloses cytoplasm of the secreting cell $[6,41]$ and range from $30 \mathrm{~nm}$ to $1,000 \mathrm{~nm}$. Extracellular vesicles can be classified into two major types: (1) vesicles of endosomal origin, released into the extracellular space by exocytosis are generally called exosomes and (2) vesicles that derive directly from the plasma membrane of the cell are generally referred to as ectosomes [42]. Many other names like, microvesicles, microparticles, oncosomes, exosome-like vesicles, or shedding vesicles are used in the 
literature as well, referring to the EVs size, cell or tissue of origin or to their suggested function $[6,42]$.

\section{Biogenesis of exosomes and ectosomes}

In general, the formation of exosomes and ectosomes shows a similar mechanism. Components are sorted and accumulated in small domains in the membrane of origin, which then undergo budding and separate from the membrane (Fig. 1.2) [6, 42]. Finally, both are released into the extracellular space $[6,41,42]$, although the molecular machineries involved in the sorting, formation and release most likely differ [6, 41, 42].

Biogenesis of exosomes The endocytotic pathway involves internalization of proteins of the plasma membrane, which results in the formation of early endosomes. While early endosomes mature into late endosomes intraluminal vesicles (ILVs) are formed and accumulate inside the late endosomes [6, 41, 42]. ILVs are formed by inwards budding of the early endosome membrane [6, 41, 42], ranging from $30 \mathrm{~nm}$ to $100 \mathrm{~nm}$ in size. Proteins, lipids and cytosol are specifically sorted into the ILVs during their formation $[6,41,42]$. These late endosomes, loaded with ILVs are called multivesicular bodies (MVBs) [6, 41, 42]. Multivesicular bodies either fuse with lysosomes, which leads to the degradation of their content, or with the plasma membrane, releasing their content into the extracellular space. ILVs released into the extracellular environment are then called exosomes [6, 41, 42].

The biogenesis of MVBs and their ILVs, involves multiple molecular machineries and is not yet fully understood $[6,41]$. Specific molecules accumulate in small membrane domains, which start budding inwards $[6,41,42]$. The cytosolic endosomal sorting complex required for transport (ESCRT) drives the transfer of ubiquitinate proteins from the plasma membrane and early endosome into budding ILVs [6, 41-43]. ESCRT consist of four multi-protein complexes (ESCRT-0, -I, -II and -III) and associated proteins like Alix (Programmed cell death 6-interacting protein) or VPS4 (Vacuolar protein sortingassociated protein 4) [6, 41, 42, 44]. Ubiquitinylated proteins are recognised and sequestered by the ESCRT-0 complex [6, 41, 42]. The ESCRT-I and -II complexes deform the endosomal membrane and thereby lead to initial inwards budding [6, 41, 42]. Thereafter, the ESCRT-III complex allows vesicle scission [6, 41, 42, 45]. Beside this ESCRT-depending mechanism the formation of ILVs also occurs via ESCRT-independent 


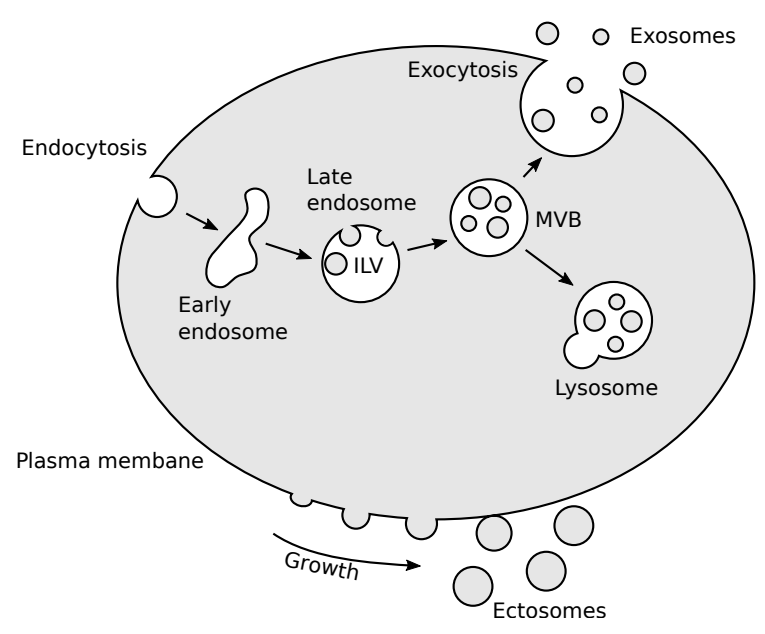

Figure 1.2: Schema of the biogenesis of exosomes and ectosomes. Exosomes and ectosomes are two types of extracellular vesicles (EVs). Exosomes are of endosomal origin. Early endosomes mature into late endosomes. Specific components are sorted into small domains of the endosomal membrane, which undergo inwards budding and finally separation from the membrane forming intraluminal vesicles (ILVs). The ILVs accumulate inside the late endosomes and thereby form multivesicular bodies (MVBs). These MVBs either follow the degradative lysosomal pathway, by fusing with lysosomes, or release their content into the extracellular space, by fusing with the plasma membrane. ILVs released into the extracellular space are called exosomes. Ectosomes derive directly from the plasma membrane. Components are sorted into small domains in the plasma membrane, which undergo outwards budding and finally pinch off into the extracellular space. The schema was altered after a figure in $[6,42]$.

mechanisms $[6,41,42]$. Trajkovic et al. first demonstrated, that exosome formation and secretion also depends on the hydrolysis of sphingomyelin to ceramide by sphingomyelinase, in an oligodendroglial cell line $[6,41,46]$.

Biogenesis of ectosomes Ectosomes directly derive from the cell plasma membrane, by outwards budding of small membrane domains [42]. Specific lipids, like cholesterol and sphingomyelin, and membrane proteins accumulate in these domains [42]. The loading of the ectosome lumen with cytosolic proteins, involves the binding of these proteins to the plasma membrane [42]. In addition, it was shown that the subunits of the ESCRT complex are also involved in the assembly and budding of ectosomes [42].

\section{Extracellular vesicle composition and intercellular communication}

Extracellular vesicles have been found in body fluids like blood [41, 47], salvia [41, 48], semen $[41,49]$, amniotic fluid $[41,50]$, urine $[41,51]$ and cerebrospinal fluid $[5,41,52]$. 
EVs are enriched with proteins, lipids and nucleic acids (mRNA, microRNA, siRNA and long noncoding RNAs) $[6,42,53]$. Although some components were found in most EVs, others are specific to the cell type of origin and the subtype of EVs, like exosomes and ectosomes $[6,42]$. Additionally, the rate of secretion was shown to vary from cell type to cell type and also to be dependent on the cells environmental conditions [6, 42].

The extracellular vesicle membrane shows an enrichment with the lipids phosphatidylserine (PS), cholesterol and sphingomyelins and their product ceramide $[6,42,54]$. The protein content of extracellular vesicles show an enrichment with specific protein families. These proteins are mainly of endosomal, cytosolic or of plasma membrane origin, while the amount of proteins from the nucleus, endoplasmic reticulum, Golgi and mitochondria is very limited $[6,42]$. The EVs membrane contains proteins of various functions: 1) Membrane transport/fusion proteins (e.g. flotillins), 2) antigen presentation (e.g. major histocompatibility complex (MHC) class I and II), 3) adhesion molecules (e.g. tetraspanins like CD63) and 4) other transmembrane proteins (e.g. lysosome-associated membrane protein (LAMPs)) [6, 42]. Furthermore, the vesicles contain endosomal sorting complex required for transport (ESCRT) components, enzymes, cytoskeletal proteins (e.g. actin and tubulin), molecules involved in signal transduction and other cytosolic proteins $[6,42]$.

Originally, seen as a cellular mechanism to discard unwanted cellular components, the discovery of EVs containing nuclei acids led to the understanding that EVs are part of the intercellular communication $[6,42,53,55,56]$. It was shown, that EV contained mRNA affects the gene expression in distant cells $[6,42,53,57]$ and that the RNA contained changes with the environmental conditions of the secreting cell. For example it was shown, that the choroid plexus within the ventricular system of the brain secretes exosomes enriched with specific nucleic acid content upon systemic inflammation [3].

After the release into the extracellular space, EVs can interact with recipient cells by direct contact or by releasing their content shortly after discharge and thereby effecting the surrounding cells $[6,42,56]$. Extracellular vesicles, which reach recipient cells, may change the physiological state of the cell by binding to components of the cell plasma membrane $[6,42,58]$, releasing their content into the cytoplasm of the recipient cell after fusion with the plasma membrane [6, 42], or by being taken up into the cell by endocytosis or phagocytosis $[6,42,59,60]$. However, the mechanisms of EVs transport towards their specific recipient cells remains mostly unknown an aspect examined in this thesis. 


\subsection{Introduction to lipids and liposomes}

Lipids have various functions within a cell, ranging from the storage of energy to compartmentalization by hydrophobic barrier formation [61, 62]. Additionally, lipids can also act as first and secondary messengers and thereby are involved in signal transduction and molecular recognition processes [61, 62]. Lipids show a large structural variety and can be categorized into eight classes: fatty acyls, prenols, glycerolipids, glycerophospholipids, sphingolipids, saccharolipids, sterol lipids and polyketides [62].

Cell membranes are generally composed of thin lipid bilayer (about $5 \mathrm{~nm}$ thick) and proteins, which are inserted into the lipid bilayer [63] (Fig. 1.3 A and B). Phospholipids represent the abundant part of biological membranes and comprise glycerophospholipids and the sphingolipid sphingomyelin [63, 64]. They consist of a hydrophilic (polar) head group and hydrophobic (nonpolar) tails, which usually are two fatty acids (Fig. 1.3 A). Hence, they are amphiphilic in their properties [63]. Aside from phospholipids the sterol lipid cholesterol and glycolipids are often part of cell membranes [63].

Within an aqueous solution phospholipids self-assemble into micelles or into lipid bilayers (Fig. 1.3 B and G). The later consist of two monolayers, the leaflets, with the hydrophobic tails facing each other and bordered by the hydrophilic head group on each side (Fig. 1.3 B). Left alone lipid bilayers tend to close in on themselves and thereby form separated compartments e.g. vesicles $[63,64]$. The molecular shape of the lipids influences the packing of the lipids within the bilayer and therefore their properties. Thereby, an important role is played by the lipid head group [10, 61].

The mammalian cell membrane predominately consists of the glycerophospholipids phosphatidylcholine (PC), phosphatidylserine (PS) and phosphatidylethanolamine (PE), the sphingolipids sphingomyelin and the sterol cholesterol [61, 63, 65]. Glycerophospholipids have a glycerol backbone with two long-chain fatty acids and a phosphate group, which can be linked to one of several head group substituents [63, 66] (Fig. 1.3 C). Depending on the head-group the glycerophospholipids carry a net charge and influence the curvature of the lipid bilayer. Lipids with a small head group in comparison to their hydrophobic tails have a conical-like shape and induce negative curvature (Fig. 1.3 E) [64, 66, 67]. An example for a conical shaped lipid is PE. Lipids, such as lysophospholipid, with a large head group in comparison to their hydrophobic part have an invertedconical shape (Fig. 1.3 E) [64, 66, 67]. These lipids induce positive curvature to a lipid bilayer and form structures such as micelles. Cylindrical shaped lipids like PC tend to 
from flat lipid bilayers (Fig. 1.3 E) [64, 66, 67]. The manner in which lipids are distributed over the cell membrane defines its form and specifies its properties. In eukaryotic cells the types and amounts of lipids found in the two leaflets of a membrane usually differ from each other [67]. This is called trans-lipid asymmetry [67, 68]

\subsubsection{Formation and classification of liposomes}

Liposomes are small self-enclosed spherical or oval lipid vesicles composed of at least one lipid bilayer and were first described by Bangham et al. in the mid 60s [9, 10, 69]. The lipid bilayer separates the internal volume, filled with solvent, and the external medium $[9,70]$. Based on the number of lipid bilayers liposomes are distinguished between multilamellar vesicles (MLV), which consist of multiple bilayers formed over each other and unilamellar vesicles, consisting of one lipid bilayer $[9,10,70,71]$. Unilamellar vesicles are classified into small unilamellar vesicles (SUVs) and large unilamellar vesicles (LUVs) (Fig. 1.3 F) [70-72]. The properties of liposomes depend on the lipid composition, the resulting surface charge, the liposome size and are crucial for their interaction with living cells $[10,73]$. Depending on the application, various methods can be used for the preparation of liposomes [10, 70, 74], which mostly involve: 1) mixing lipids in an organic solvent and drying down the lipids from the solvent, 2) dispersing the lipid in aqueous solvent and 3 ) the purification (e.g. removing a detergent used during the previous step) and analysis (e.g. measuring the size distribution) of the produced liposomes [10].

Liposomes are well established as model to study cell membranes, their fusion, the transport and behavior of membrane proteins $[9,10]$. Furthermore they are used as delivery systems of drugs, genetic material, enzymes and other molecules into living cells $[9,10,75,76]$ and thus appear to be the optimal tool to mimic extracellular vesicles in solution. 
A

$$
\Gamma^{7} \begin{aligned}
& \text { hydrophilic } \\
& \text { head } \\
& \text { hydrophobic } \\
& \text { tails }
\end{aligned}
$$

\section{B}

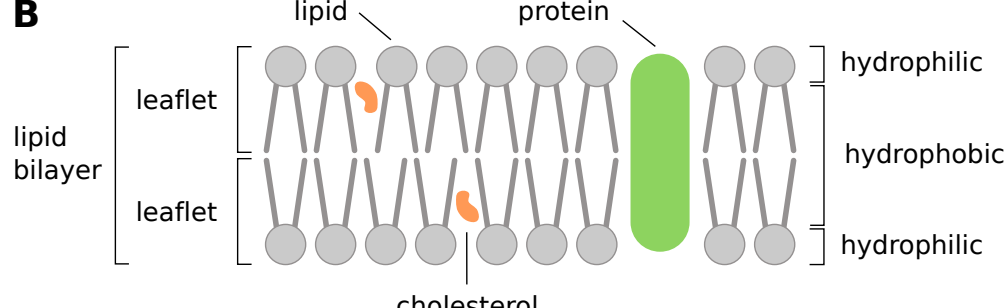

cholesterol

\section{Glycerophospholipids}

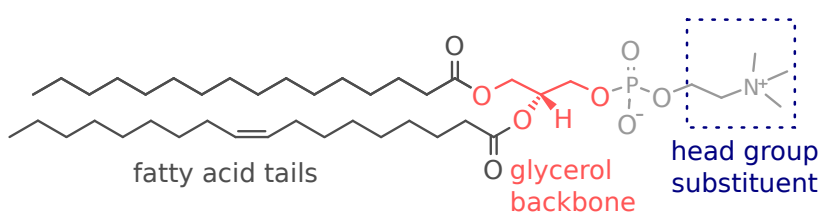

E Membrane curvature

Lipids:

PE

PA $(\mathrm{pH}<3)$

PS $(\mathrm{pH}<3)$

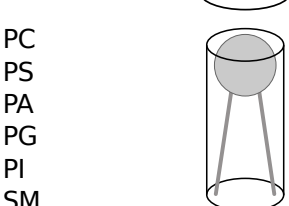

SM

Lysophospho-
lipids

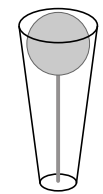

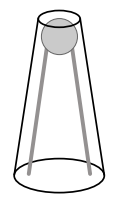

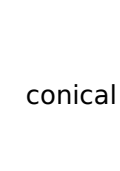

cylindrica

invertedconical

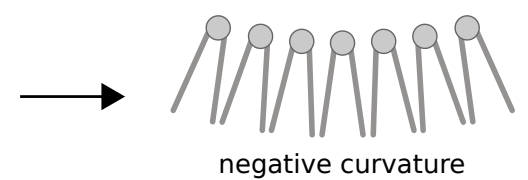

negative curvature

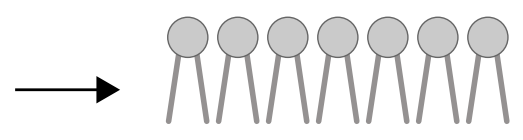

flat membrane

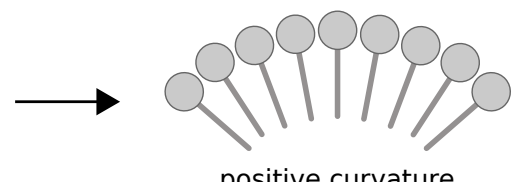

D Cholesterol

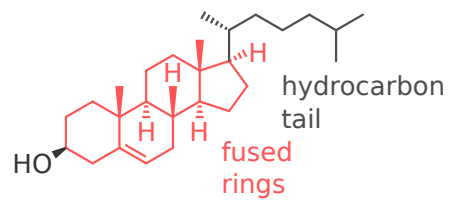

F Liposome

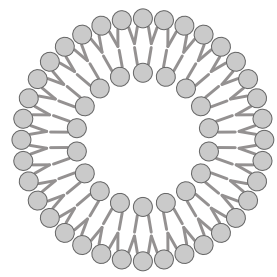

G Lipid micelle

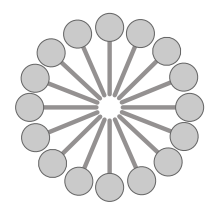

Figure 1.3: Cell membrane lipids and self-assembly behavior. A Schema of an amphiphilic lipid with a hydrophilic head group and hydrophobic hydrocarbon tails [63]. B Schema of a cell membrane composed of a lipid bilayer and proteins. The lipid bilayer consists of two lipid monolayers, the leaflets [63]. C Glycerophopholipids have a glycerol backbone with usually two long fatty-acid chains and a phosphate group. One of several head group substituents can be bound to the phosphate group forming the lipids head-group $[63,66]$. The example shown is phosphatidylcholine (PC). D Schema of the sterol cholesterol [66]. Many membranes contain cholesterol in addition to phospholipids. $\mathbf{E}$ The molecular shape of a lipid influences the membrane curvature and bends the membrane [64, 66]. Phosphatidylethanolamine (PE), phosphatidic acid (PA), phosphatidylserine (PS), phosphatidylcholine (PC), phosphatidylglycerol (PG), phosphatidylinositol (PI), sphingomyelins (SM). F Schema of a unilamellar liposome. G Lipids with an inverted-conical shape tend to form micelles in aqueous solutions. 


\section{Chapter 2}

\section{Materials and Methods}

\subsection{Collaborations}

The cilia-generated flow analysis was performed in collaboration with the Max Planck Institute for Dynamics and Self-Organisation. The interaction of fluorescent liposomes and fluores-cently-labeled EVs with ciliated epithelia was studied in collaboration with Dr. Regina Faubel from Dr. Cecilia Lo's lab at the University of Pittsburgh School of Medicine.

\subsection{Animals}

\subsubsection{Animal housing and breeding}

All animal experiments were carried out in compliance with the German Law on Animal Welfare and were approved by the Office for Consumer Protection and Food Safety of the State of Lower Saxony. The mice were housed in transparent, individually ventilated cages with filter tops in specific pathogen free conditions. Room temperature and humidity were kept constant at $21^{\circ} \mathrm{C}$ and $55 \%$, respectively, and animals were kept in a $12 \mathrm{~h}$ light $12 \mathrm{~h}$ dark cycle with lights on at 6 a.m. Standard chow and water were provided ad libitum. Breeding was established in pairs or triplets (two females and one male), after the mice reached an age of eight weeks. The pups were weaned at the age of three weeks.

All experiments performed in the lab of Dr. Cecilia Lo at the University of Pittsburgh were carried out in compliance with the DLAR. 


\subsubsection{Circadian entrainment of animals}

For circadian entrainment C57BL/6NJ (B6N) wildtype mice at the age of eight weeks were placed in separate entrainment boxes. The entrainment boxes stood in a dedicated room and were impervious to light. Each entrainment box provided space for one cage and was connected to the ventilation system. Two to five animals were housed per cage. The mice were entrained to a $12 \mathrm{~h}$ light : $12 \mathrm{~h}$ dark cycle (LD 12:12) for two weeks at a light intensity of $450 \mathrm{~lx}$ in the light phase and $0.0 \mathrm{~lx}$ in the dark phase.

Mice show the highest level of activity during the dark phase and rest during the light phase. The entrainment boxes allowed to entrain the animals independently of the environmental diurnal rhythm. Entrainment times were chosen in a way, that the animals were not perturbed by noises from the environment during the light phase.

All mice were analyzed under Zeitgeber time (ZT) conditions. ZT10 is defined as, $10 \mathrm{~h}$ after the light was switched on. ZT23.5 is in the dark phase, $23.5 \mathrm{~h}$ after light was switched on. The brain of mice were isolated under red light, when the animals were in the dark phase and under light, when the animals were in their light phase. The mice were sacrificed by cervical dislocation.

\subsection{Vesicle preparation for cilia logistic network characterization}

\subsubsection{Chemicals and Reagents}

1,2-dioleoyl-sn-glycero-3-phosphocholine (DOPC, 850375), 1,2-dioleoyl-sn-glycero-3phosphoethanolamine (18:1 ( $\Delta 9$ - Cis) DOPE, 850725), 1,2-dioleoyl-sn-glycero-3-phospho-L-serine (18:1 DOPS, 840035) and cholesterol (ovine, 700000) were brought from Avanti Polar Lipids, USA. Head group labeled DOPE with ATTO488, ATTO550 and ATTO647N were obtained from AttoTec, Germany. Fatty acid labeled 1-Oleoyl-2-[12[(7-nitro-2-1,3-benzoxadiazol-4-yl)amino]dodecanoyl]-sn-Glycero-3-Phosphocholine (18:1 12:0 DOPC-NBD, 810133) was brought from Avanti Polar Lipids, USA. All lipid stocks were dissolved in chloroform and stored in brown capped bottles (Carl Roth, Germany) at $-20^{\circ} \mathrm{C}$.

mCLING-ATTO647N (membrane-binding fluorophore-cysteine-lysine-palmitoyl group) was brought from Synaptic Systems, Germany. All lipids were solved in chloroform obtained from Merck KGaA Germany. mCLING was dissolved in dimethyl sulfoxide (DMSO, Sigma, Germany). 
Liposomes were prepared by size exclusion chromatography using an Econo-Column of $0.7 \mathrm{~cm}$ diameter and $15 \mathrm{~cm}$ length from BioRad, Germany. Sephadex G5050 superfine obtained from Sigma Aldrich, Germany. Further, liposomes were prepared using a MiniExtruder from Avanti Polar Lipids, USA, with polycarbonate membrane of $0.1 \mu \mathrm{m}$ pore size (Nuclepore Track-Etched Membranes 800309 19MM, Whatman) and $0.03 \mu \mathrm{m}$ pore size (Nuclepore Track-Etched Membranes 800307 19MM, Whatman).

Liposomes were prepared in HP-150 buffer containing HEPES obtained from Sigma Aldrich, Germany and potassium chloride bought from Merck KGaA, Germany. Sodium cholate hydrate was purchased from Sigma Aldrich, Germany. Ethanol (absolute, for analysis) was bought from Merck KGaA, Germany. Phosphate-buffered saline was prepared with sodium-chloride from Merck KGaA, Germany and disodium-hydrogen-phosphate obtained from Sigma Aldrich, Germany. All buffers were prepared in Milli-Q water produced by a Millipore Crop., USA filter system.

\subsubsection{Preparation of fluorescently-labeled liposomes}

Liposomes can be prepared by several methods [10,74]. All methods involve the drying down of a lipid mixture from an organic solvent and the following reconstitution of the dried lipid film in an aqueous medium [10, 74]. Here, fluorescently-labeled liposomes were prepared by two methods: (1) The dried lipid film was reconstituted in an aqueous detergent containing buffer and processed further by size exclusion chromatography $[10$, 74]. (2) After reconstitution in detergent free aqueous buffer, liposomes were processed by extrusion $[10,74]$. Both methods were used to produce small liposomes formed of one lipid bilayer, so called small unilamellar vesicles (SUV).

\section{Preparation of liposome stock solution}

Lipids, including cholesterol, dissolved in chloroform, were mixed in a clean glass flask using Hamilton syringes. After the addition of a particular lipid stock, the Hamilton syringes were cleaned three times with chloroform. Lipid stocks were kept on ice at all times. The glass flask was wrapped in aluminium-foil, to protect fluorescently-labeled lipids from light. In case liposomes were to be labeled with mCLING, the mCLING crystalline powder was dissolved in DMSO and added directly to the lipid-mixture.

Next, the chloroform was removed to yield a lipid film. For small sample volumes under $0.5 \mathrm{~mL}$ the solvent was removed by evaporation under a dry nitrogen stream in a 
fume hood. During evaporation the flask was slowly rotated by hand. Afterwards the flask with the lipid film was placed in a desiccator for three hours. In case of large sample volumes, the solvent was removed by using a rotary evaporator.

HP-150 buffer containing 20mM HEPES, $150 \mathrm{mM}$ potassium chloride and 5\% sodium cholate (detergent) was freshly prepared with a $\mathrm{pH}$ of 7.4. The dried lipid film was hydrated by adding HP-150 buffer. Then, the glass flask was vortexed until the lipid film was completely dissolved. The lipid mixture was aliquoted as $25 \mu \mathrm{L}$ aliquots and stored at $-20^{\circ} \mathrm{C}$ until use.

\section{Size exclusion chromatography}

Econo-Column was washed with Hellmanex (a cleaning concentrate obtained from Sigma-Aldrich, Germany) and flushed four times with 100\% ethanol, one time with $70 \%$ ethanol and five times with Milli-Q water. Then, the column was filled with filtered HP150 buffer without cholate. $0.45 \mathrm{~g}$ of sephadex G5050 superfine were mixed in $7 \mathrm{~mL}$ filtered HP-150 and allowed to swell for $15 \mathrm{~min}$. After that, the sephadex-matrix was pipetted into the HP-150 filled column and allowed to pack until the matrix was completely settled down. The excess buffer on top of the matrix was allowed to drip into the matrix. After all the buffer had lowered into the matrix, the lipid-mixture stock solution was added on top. Next, the lipid-mixture was seeped into the matrix completely, HP-150 buffer was carefully added on top until the column was filled. The fluorescently-labeled lipid mixture could be observed as a coloured band while going down the column. In case of mCLING containing lipid mixture the band was not clearly visible. Scattered light from a laser pointer was used to determine liposomes in the eluate. The eluate was collected and stored at $4{ }^{\circ} \mathrm{C}$. Liposomes were used up to $48 \mathrm{~h}$ after preparation.

\section{Extrusion}

As described above, Lipids and cholesterol, dissolved in chloroform, were mixed in a clean glass flask using Hamilton syringes. Afterwards the chloroform was evaporated under a dry nitrogen stream in a fume hood. During the process the flask was slowly rotated by hand. Then the flask was then placed in a desiccator for three hours. HP150 buffer (20 mM HEPES, $150 \mathrm{mM}$ potassium chloride) was freshly prepared without cholate. $250 \mu \mathrm{L}$ of HP-150 buffer were added to the lipid film and the glass flask was vortexed until the lipid film was completely dissolved. 
The Mini-Extruder was cleaned with ethanol and Milli-Q water before use and prepared according to manufactures instructions. The liposome solution was extruded 11 times through a $0.1 \mu \mathrm{m}$ pore size polycarbonate membrane. Then, the resulting liposome solution was extruded again 31 times through a $0.03 \mu \mathrm{m}$ pore size polycarbonate membrane. The obtained liposome solution was stored at $4{ }^{\circ} \mathrm{C}$ until use.

\subsubsection{Compositions of fluorescently-labeled liposomes}

Living cells contain a high diversity of lipids $[66,77]$. The plasma membrane mainly consists of glycerophospholipids, sphingolipids and sterol [66, 77]. In mammalian cells the glycerophospholipids phosphatidylcholine (PC), phosphatidylethanolamine (PE) and phosphatidylserine (PS), as well as the sphingolipids sphingomyelin and the sterol cholesterol are predominant $[65,77]$. The liposome lipid composition I, II, VII and VIII (Tab. 2.1 and 2.2) were prepared mimicking this lipid mixture.

Liposomes were prepared by size exclusion chromatography and extrusion with varying lipid compositions and dyes. The liposome stock solutions, which were further processed by size exclusion chromatography were all prepared with a total lipid concentration of $30 \mathrm{mM}$ after hydration with HP-150 buffer containing 5\% cholate. All compositions used are listed in Table 2.1.

Liposome solutions, further processed by extrusion, were prepared with a total lipid concentration of $12 \mathrm{mM}$ and hydrated with HP-150 buffer without cholate. The prepared compositions are listed in Table 2.2. 


\begin{tabular}{l||ccccccc}
\multicolumn{1}{c||}{} & \multicolumn{7}{c}{ Compositions } \\
Components & I [\%] & II [\%] & III [\%] & IV [\%] & V [\%] & VI [\%] & VII [\%] \\
\hline \hline DOPC & 50.00 & 50.00 & 70.00 & 60.00 & 48.50 & 88.5 & 50.97 \\
DOPS & 20.00 & 20.00 & - & 20.00 & 20.00 & - & 20.00 \\
DOPE & 18.50 & 18.50 & 18.50 & 18.50 & 20.00 & - & 19.00 \\
Cholesterol & 10.00 & 10.00 & 10.00 & - & 10.00 & 10.00 & 10.00 \\
DOPE-ATTO550 & 1.50 & - & - & - & - & - & - \\
DOPE-ATTO647N & - & 1.50 & 1.50 & 1.50 & - & - & - \\
NBD-PC & - & - & - & - & 1.50 & 1.50 & - \\
mCLING-ATTO647N & - & - & - & - & - & - & 0.02
\end{tabular}

Table 2.1: List of components used for liposomes prepared by size exclusion chromatography. The lipids DOPC (1,2-dioleoyl-sn-glycero-3-phosphocholine), DOPS (1,2-dioleoyl-sn-glycero3-phospho-L-serine), DOPE (1,2-dioleoyl-sn-glycero-3-phosphoethanolamine) and cholesterol were used in varying amounts. Liposomes were marked with head group labeled DOPE, fatty acid labeled PC or mCLING (membrane-binding fluorophore-cysteine-lysine-palmitoyl group). The composition rows give the percentage of each lipid in the resulting membrane (\%).

\begin{tabular}{l||cc}
\multicolumn{1}{c||}{} & \multicolumn{2}{c}{ Compositions } \\
\multicolumn{1}{c||}{ Components } & VIII [\%] & IX [\%] \\
\hline \hline DOPC & 20.00 & 98.50 \\
DOPS & 20.00 & 0.00 \\
DOPE & 18.50 & 0.00 \\
Cholesterol & 10.00 & 0.00 \\
DOPE-ATTO647N & 1.50 & 1.50
\end{tabular}

Table 2.2: List of components used for liposomes prepared by extrusion. The lipids DOPC (1,2dioleoyl-sn-glycero-3-phosphocholine), DOPS (1,2-dioleoyl-sn-glycero-3-phospho-L-serine), DOPE (1,2-dioleoyl-sn-glycero-3-phosphoethanolamine) and cholesterol were used in varying amounts. Liposomes were marked with head group labeled DOPE. The composition rows give the percentage of each lipid in the resulting membrane (\%). 


\subsubsection{Measurement of liposome concentration and size distribution}

The size distribution of freshly prepared liposome samples was analyzed by electron microscopy and nanoparticle tracking analysis (NTA). Electron microscopy was performed in the institutional facility headed by Dr. Dietmar Riedel. The liposome samples were prepared for electron microscopy using a negative staining. The images were analyzed using Fiji [78].

NTA was conducted using a NanoSight NS300 from Malvern Panalytical equipped with a 405 laser module. The liposome sample was diluted in a ratio of 1:500, 1:1000 and 1:2000 in HP-150 buffer. For each dilution, a series of five measurements was made and analyzed with NanoSight software version 3.2. As control, pure HP-150 buffer was measured with the same parameters. In addition, NTA allowed to estimate the concentration of particles per $\mathrm{mL}$ for each sample.

\subsubsection{Preparation of extracellular vesicles}

All protocols for isolation, controls and labeling of extracellular vesicles were established, optimized and performed by Dr. Zuzana Ditte in the Eichele laboratory (Max Planck Institute for Biophysical Chemistry).

\section{Chemicals and Reagents}

Dublecco's modified essential medium (DMEM), Dulbecco's Phosphate-Buffered Saline (DPBS), Fetal bovine serum (FBS) and antibiotic (Penicillin Steptomycin 100 units $/ \mathrm{mL}$ ) were obtained from Gibco, Thermo Fisher Scientific, Germany. Polyethylene glycol with $\mathrm{M}_{n}$ 6,000 was bought from Sigma Aldrich, Germany and TRIZOL reagent from Life Technologie (Thermo Fisher Scientific), Germany. A Econo-Column of $0.7 \mathrm{~cm}$ diameter and $15 \mathrm{~cm}$ length from BioRad, Germany was used for size exclusion chromatography in combination with sephadex superfine G5050 obtained from Sigma Aldrich, Germany.

Quantitect Reverse Transcription Kit was obtained from Qiagen and KiCqStart Universal SYBR Green qPCR Ready Mix from Sigma Aldrich, Germany. Goat serum was bought from Vectors laboratories.

The following primary antibodies were used: Alix-monoclonal of mouse origin obtained from Thermo Fischer Scientific, Germany, Flotillin 2 of mouse origin bought from BD Transduction Laboratories, Germany, $\beta$-catenin of rabbit origin obtained from Sigma 
Aldrich, Germany, CD63-polyclonal of rabbit origin from Sigma Aldrich, Germany and AGO-2-monochlonal of rat origin from Sigma Aldrich.

The following secondary antibodies were used: Alexa Fluor 488 conjugated goat antimouse IgG (Life Technologies) and Alexa Fluor 555 conjugated anti-rabbit IgG (Invitrogen) were bought from Thermo Fisher Scientific, Germany. Goat anti-rabbit IgG, HRP-conjugate was obtained from Novex, goat anti-mouse-HRP from Jackson Immuno Research Laboratories and goat anti rat from Dianova.

\section{Cell Culture}

Z310 rat choroid plexus cell line Immortalized Z310 choroidal epithelial cell line from murine choroid plexus was gifted to the lab by Prof. R. Steinfeld of the Universitätsmedizin Göttingen. The cell line was originally created from choroid plexus tissue collected from Sprague Dawley rats of both sexes at an age of 4 to 6 weeks [79].

The cells were grown in DMEM supplemented with $10 \%$ of FBS, 100 units $/ \mathrm{mL}$ antibiotic in humidified incubator with $95 \%$ air, $5 \% \mathrm{CO}_{2}$ at $37^{\circ} \mathrm{C}$. The cells were passaged twice a week and were regularly checked for mycoplasma contamination.

NIH-3T3 cell line The NIH-3T3 (OMEGA-E) cells were established from Swiss mouse embryo and grow as adherent fibroblast monolayers. The cells were obtained from Leibniz Institute DSMZ-German Collection of Microorganisms and Cell Cultures.

The cells were grown in DMEM supplemented with $10 \%$ FBS, 100 units $/ \mathrm{mL}$ antibiotics in a humidified incubator with $95 \%$ air, $5 \% \mathrm{CO}_{2}$ at $37^{\circ} \mathrm{C}$. The cells were passaged every three to four days.

\section{Collecting extracellular vesicles}

The cells were cultured in serum-free medium - conditioned media $(\mathrm{CM})$ - , to reduce contamination from serum proteins. The cells were monitored daily for changes in growth rate and the proportion of live to dead cells to minimize production of apoptotic bodies in addition to extracellular vesicles.

Harvested media from multiple flasks were pooled for each experiment. CM was collected and centrifuged at $10,000 \times \mathrm{g}$ for $10 \mathrm{~min}$ to remove cell debris and larger vesicles. Next, the CM was transferred to a new tube and centrifuged at $10,000 \times \mathrm{g}$ for $30 \mathrm{~min}$ 
to remove larger micro-vesicles. Then samples were processed by two different isolation methods, size exclusion chromatography $(\mathrm{SECH})$ and precipitation of EVs based on polyethylene glycol (PEG).

Size exclusion chromatography (SECH) The CM was concentrated using a 300,000 MWCO VivaSpin 20 Centrifugal Concentrator (Sartorius, Germany) and applied to a Econo-Column of $0.7 \mathrm{~cm}$ diameter and $15 \mathrm{~cm}$ length from BioRad, Germany. The column was packed with sephadex G5050 equilibrated with phosphate buffered saline (PBS). The samples were eluted with PBS and $1 \mathrm{~mL}$ fractions were collected. The protein content of each fraction was monitored by measuring the absorbance at $280 \mathrm{~nm}$, using a ND1000 Spectrophotometer (NanoDrop). The fractions were concentrated using 300,000 MWCO VivaSpin 6 Centrifugal Concentrators (Sartorius, Germany) by centrifugation at $4,000 \times \mathrm{g}$ for 5 to $10 \mathrm{~min}$, depending on the content.

Precipitation of EVs using PEG Polyethylene glycol solution was added to CM to a final concentration of $10 \%$ and thoroughly mixed by inversion. The solution was incubated overnight at $4{ }^{\circ} \mathrm{C}$. The next day, the samples were centrifuged at $10,000 \times \mathrm{g}$ for $30 \mathrm{~min}$ and resuspended in PBS.

\section{Labeling of EVs}

Extracellular vesicles were obtained from Z310 rat choroid plexus cells by SECH or precipitation with PEG. Afterwards, EVs were labeled with primary antibodies against Alix or Flotillin 2 and secondary antibody Alexa Fluor 488 anti-rabbit IgG resp. anti-mouse IgG. Then EVs were monitored by fluorescence microscopy and stored at $4{ }^{\circ} \mathrm{C}$ until use.

\section{Characterization of EVs}

Previous, protein analysis of extracellular vesicles has highlighted commonly found proteins in EV preparations. It has become clear, that these proteins do not represent exosomespecific markers, but rather exosome-enriched proteins [80]. It is also well known that in different types of EVs relative proportions of different proteins seems to vary [80].

Based on these findings, the International Society for Extracellular Vesicles advised, that EVs should be characterized and described by proteins expected to be present in the 
EVs of interest. Especially transmembrane proteins and cytosolic proteins with membranebinding capacity like CD63 and Alix. In addition, the presence of proteins of endosomal origin, which are not expected to be enriched in EVs, should be determined as well, for example Ago and Golgi [80].

The Z310 rat choroid plexus cells and isolated extracellular vesicles were characterized by western blotting using CD63, Alix, Ago 2 and Flotillin 2 as markers. Further, EVs samples were analyzed by nano-particle tracking analyses and electron microscopy, performed by the institutes electron microscopy facility. Additionally, the mRNA of Ago 2 and Golga 2 expression was compared between Z310 cells and EVs.

Western blotting For western blotting, samples (EVs or cell lysates) were boiled in Laemmli sample buffer (Sigma Aldrich, Germany) under non-reducing conditions for CD63 blots and reducing conditions for all others markers. Afterwards the samples were subjected to $10 \%$ tris-glycine SDS-PAGE and then transferred to PVDF membranes (Bio Rad, Germany). Membranes were blocked with $5 \%$ milk powder in TBST $(0.1 \%$ Tween 20 in TBST) for $1 \mathrm{~h}$, followed by incubation with the corresponding primary antibody overnight at $4{ }^{\circ} \mathrm{C}$ in blocking buffer. Membranes were washed and then incubated with HRP-conjugated secondary antibody goat anti-rabbit resp. anti-mouse for $1 \mathrm{~h}$ at room temperature. The membranes were washed three times in TBST with $0.1 \%$ Tween. The blots were developed using ECL SuperSignal West Femto (Thermo Fisher Scientific, Germany) and visualized by ImageQuant LTAS4000.

RNA isolation The total RNA from cells and EVs was extracted using TRIZOL reagent (Life Technologie) according to the manufacture's instructions. The quality of RNA (A260/ A280) was about 2.0 for all RNA preparations.

cDNA synthesis and qPCR For cDNA synthesis, the Quantitect Reverse Transcription Kit (Qiagen, Germany) was used according to the manufacturer's descriptions, using $12 \mathrm{ng}$ of RNA. The Real-Time qRT-PCRs were performed using the KiCqStart Universal SYBR Green qPCR Ready Mix (Sigma-Aldrich, Germany) according to manufacturer's protocol. $100 \mathrm{ng}$ of cDNA was used per reaction. For amplification and detection, the Biorad CFX96 was used. All data were normalized to the expression levels of $18 \mathrm{~s}$ rRNA, U6 and GADPH. 


\section{Wound closure assay: Comparing liposomes and EVs from Z310 cells}

NIH 3 T3 cells were plated on glass coverslips for $24 \mathrm{~h}$ at $37^{\circ} \mathrm{C}$ to become 90 to $100 \%$ confluent the next day. $200 \mu \mathrm{L}$ pipette tip was used to make a vertical wound down through cell monolayer. Then cell debris were carefully removed by washing with PBS and the uptake experiment was performed by incubating the cell cultures with liposomes or EVs for $30 \mathrm{~min}$ at $37^{\circ} \mathrm{C}$ and $5 \% \mathrm{CO}_{2}$. Medium was removed and cells were washed twice with PBS and fixed with $4 \%$ paraformaldehyde. Next, samples were washed three times with solution containing $0.1 \%$ BSA and $0.1 \%$ Tween in PBS, and blocked for 30 min with $10 \%$ normal goat serum in PBS.

First, coverslips were incubated overnight at $4{ }^{\circ} \mathrm{C}$ with primary antibody, $\beta$-catenin in a solution containing $0.25 \%$ BSA, $0.25 \%$ Tween in PBS. The next day, the cells were washed three times with a solution containing $0.1 \%$ BSA and $0.1 \%$ Tween in PBS. Then, the cells were incubated with secondary antibody Alexa Fluor 555 conjugated goat antirabbit $\operatorname{IgG}(0.1 \% \mathrm{BSA}$ and $0.1 \%$ Tween in PBS) for $1 \mathrm{~h}$ at room temperature, washed three times with PBS and mounted on glass slides in mounting medium containing DAPI (VECTASHIELD). Unwounded cells were processed as control in the same way.

Afterwards, the cells were observed by fluorescence microscopy using a OLYMPUS IX81 microscope equipped with a FluoView FV1000 confocal laser scanning system. All hardware was operated by OLYMPUS FV10-ASW Version 4.2 software. Image stacks were taken with 10x (UPLFL 10x 0.30), 40x (U PLSAPO 40x/0.90) or 60x (U PLSAPO 60x O 1.35) magnification.

\subsection{Analysis of cilia-generated flow in the ventral third ventricle}

The preparation of the ventral third ventricle for cilia-generated flow analysis was developed and described in detail by Dr. Regina Faubel [2, 81]. The flow map analysis was developed by Dr. Christian Westendorf (MPI for Dynamics and Self-Organisation), based on a particle tracking algorithm designed by Nicholas Darnton and Jake Jaffe [82] ${ }^{1}$.

\subsubsection{Preparation of the ventral third ventricle: open pouch}

A fresh mouse brain was dissected from the skull and placed in a petri dish filled with Dulbecco's modified eagle medium (DMEM 21063, ThermoFisher Scientific, Germany)

\footnotetext{
${ }^{1}$ http: //www.rowland.harvard.edu/labs/bacteria/software/index.php
} 
to wash away remaining blood. Afterwards the brain was placed in a coronal matrix (S92, PLANO) with the ventral side facing upwards. The optical nerves were cut until the optic chiasma. Two razor blades were placed in four-millimeter distance from each other starting anterior of the optical chiasma and simultaneously pressed down. Four-millimeter coronal section, which contains the third ventricle, was taken out of the coronal matrix and placed in a new petri dish filled with DMEM.

The coronal section was held by tweezers, which were placed parallel to the third ventricle. The optical chiasma and ventral connection of the third ventricle was cut open carefully using thin tungsten needles. After that the anterior wall of the ventral third ventricle and the anterior commissure were cut. Afterwards the v3V was spread open slightly and the two hemispheres carefully separated along the entire anterior to posterior extent. Two cuts were made parallel to the midline in a distance of around $200 \mu \mathrm{m}$ from the ventricular lining. Subsequently each ventricular half was separated from the coronal section using tweezers. Only surrounding brain parenchyma but not ependyma was touched with tweezers.

\subsubsection{Imaging cilia-generated flow in the ventral third ventricle}

A MilliCell cell culture insert was placed in a small petri dish. $1 \mathrm{~mL}$ DMEM (21063, Ther-

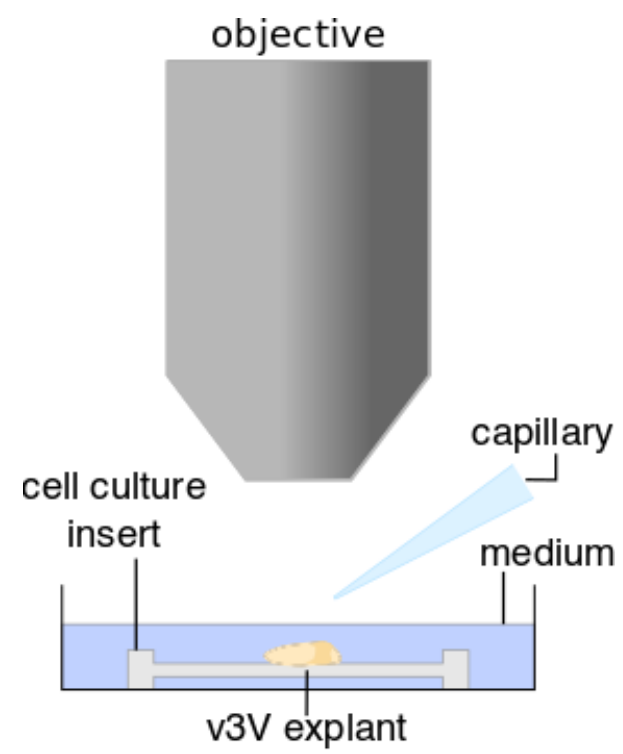

Figure 2.1: Set-up for v3V ciliagenerated flow imaging and local liposome application. A v3V explant was placed on a cell culture insert in a petri dish filled with medium (DMEM). Fluorescent beads were added and their movement along the cilia-generated flow recorded at $5 \mathrm{x}$ magnification. Local application of fluorescently-labeled liposomes using a capillary was recorded at $5 \mathrm{x}$ and $10 \mathrm{x}$ magnification. moFisher Scientific, Germany) was filled underneath and on top of the insert. Two microliters of $1 \mu \mathrm{m}$ multi-fluorescent latex beads (Fluoresbrite Multifluorent, Polyscience Inc.) were added on top of the insert and the petri dish slightly shaken. Freshly prepared v3V explant was transferred on top of the insert, with the ciliated side facing upwards (Fig. 2.1). The ventricular ependyma was not touched during transfer. 
Fluorescent beads were transported along the cilia-generated flow. Beads transported close to the ependymal wall represent the cilia-generated near wall flow, while bead trajectories in greater distance can differ from this. It was ensured that the near wall flow was imaged for every area of the v3V by using stationary beads stuck on the apical site of the ependymal cells as markers. The cilia-generated bead flow was recorded for $20 \mathrm{~s}$ with 29 fps. Furthermore, a bright-field image was taken for later localization of the region of interest (ROI).

The cilia-generated bead flow was visualized by using an upright Leica DMR microscope with mercure-vapour-lamp. Movies of the bead flow were made using a Cascade II camera (Photometrics) operated with MultiRecorder software (Johannes SchröderSchetlig, MPI for Dynamics and Self-Organization, Göttingen) at 5x (HC PL Fluostar $5 \times / 0.15$, Leica) magnification with a tube factor of 1.25 .

\subsubsection{Flow maps: Analysis of cilia-generated flow}

The cilia-generated flow pattern across the entire v3V was analyzed in two ways: 1) Particle tracking analysis was used to generate color-coded flow maps, which show the flow direction of each analyzed particle in addition to the flow pattern, 2) Maximum intensity projection was used to generate maps of the flow pattern only. The first method was applied in each experiment regarding liposome propagation within the cilia-generated flow. The second method was used in each experiment regarding the attachment of liposomes to ependymal cells. In addition, the transport velocity of fluorescent beads along the cilia-generated near wall flow was calculated for each liposome propagation experiment individually.

\section{Particle tracking analysis}

The recorded movies of cilia-generated bead flow were analyzed using Matlab (Mathworks) to generate an overall flow map of the cilia near wall flow in the v3V. Every movie consisted of grey scale images. A band pass filter was applied to all images for background correction. The band pass filter is based on the bpass filter written by Prof. David G. Grier 1993 and was modified by Dr. Christian Westendorf. Next, the images were converted to binary images based on a threshold. For all remaining single objects, the $\mathrm{x}$ - and $\mathrm{y}$ - position was calculated and fed to the particle tracking algorithm. The particle tracking algorithm was developed by Nick Darnton and Jake Jaffe [82]. Stationary beads were 
excluded from analysis. Afterwards the calculated bead trajectories were plotted with respect to their angles and directions of movement. In addition, the velocity was computed for each trajectory. The flow map across the entry $\mathrm{v} 3 \mathrm{~V}$ was generated by extraction of the near wall flow and stitching each part together.

\section{Maximum intensity projection}

The recorded movies of the cilia-generated bead flow were imported as image stacks in Fiji [78]. The image stacks were projected using maximum intensity projection. Contrast and brightness of the projection image was adjusted and the image saved. Afterwards a global flow map across the entire $\mathrm{v} 3 \mathrm{~V}$ was generated by stitching the accurate near wall flow of each projection image together.

\section{Calculation of bead transport velocity in the v3V cilia-generated near wall flow}

The velocity of beads transported along the cilia-generated near wall flow was calculated using the 2D and 3D Particle Tracker of the Fiji plugin MOSAICsuite [83, 84] and Matlab. The recorded movies of cilia-generated bead flow were cropped, so that only areas in focus, which represented the near wall flow, were included. The $2 D$ and $3 D$ Particle Tracker was used to track each particle within the given parameters. This resulted in a list of trajectories consisting of position coordinates of the particular bead in each relevant frame. From these coordinates, the bead trajectories length and velocity were calculated using Matlab. This method allowed the calculation of bead transport velocities along the same flow streams as applied liposomes only, by adjusting the area of calculation.

\subsection{Characterization of liposome distribution in the ventral third ventricle}

\subsubsection{Injection of fluorescent liposomes into cilia-generated near wall flow}

The propagation of EV-like cargo was studied by injecting fluorescently-labeled liposomes into the cilia-generated near wall flow. A v3V explant was freshly prepared before each experiment according to the description in Sec. 2.4.1. Two petri dishes were set up as described in Sec. 2.4.2. Briefly, a cell culture insert was placed in each petri dish and DMEM (21063, ThermoFisher Scientific, Germany) was added underneath and on 
top of the membrane. Two microliters of multi-fluorescent beads were added on top of the membrane and the petri dishes slightly shaken. Bead movement was used to find the exact position in the $\mathrm{v} 3 \mathrm{~V}$. The cilia-generated bead flow was recorded as described in Sec. 2.4.2.

Afterwards a glass capillary (Femtotips Type I, Eppendorf) was filled with $10 \mu \mathrm{L}$ fluorescently-labeled liposome solution and connected to FemtoJet express (Eppendorf). The capillary was brought into the exact position using a PatchMan NP 2 (Eppendorf). The distance of the capillary tip to the apical ependymal cell surface was adjusted by using fluorescent beads, which had attached on ependymal cells, as a reference.

After positioning the capillary tip, a $10 \mathrm{~s}$ movie of bead movement was recorded for later localization of the injection site and comparison of transport velocities. Fluorescentlylabeled liposomes were injected into cilia-generated near wall flow at specific locations and with varying pressure and duration. The distribution of fluorescently-labeled liposomes was recorded at 5x (HC PL Fluostar 5x/0.15, Leica) and 10x (HC PL Fluostar 10x/0.30 PH1, Leica) magnification with a tubus factor of 1.25 and 29 fps. All injection experiments were performed using a Leica DMR upright microscope with mercuryvapour-lamp.

\subsubsection{Analysis of fluorescent liposome propagation in near wall flow}

The temporal propagation of liposomes in the cilia-generated near wall flow of the $\mathrm{v} 3 \mathrm{~V}$ was visualized using Fiji's [78] temporal color code spectrum. The propagation velocity was calculated by measuring the propagation distance of injected liposomes transported close to the ependymal wall using Fiji and dividing it by the propagation time. The propagation distance was measured by tracing the liposome propagation front from frame to frame by hand, starting at the tip of the capillary along the liposome propagation lines until the near wall flow was exited. The propagation time was calculated by multiplying the frame rate with the number of frames used for tracing the liposome propagation distance.

\subsection{Interaction of liposomes with ventral third ventricle ependymal cells}

The interaction of liposomes with $\mathrm{v} 3 \mathrm{~V}$ ependymal cells was studied in terms of spatial and temporal aspects. The effect of liposome attachment to cilia was analyzed. Furthermore, 
the influence of liposomes components to liposome-cilia attachment was examined by using varying compositions of phospholipids, cholesterol and fluorescent dyes (Tab. 2.1 and 2.2).

\subsubsection{Applying liposome to open pouch preparation}

Small amounts of fluorescently-labeled liposomes were applied in cilia-generated near wall flow in flow 6 and flow 7 as described in Sec. 2.5.1. The distribution and attachment of liposomes was recorded during the injection at 5x (HC PL Fluostar 5x/0.15, Leica) and 10x (HC PL Fluostar 10x/0.30 PH1, Leica) magnification. Afterwards, the sites of liposome attachment were images at 20x (HC PL Fluostar 20x/0.50 PH2, Leica) magnification. All liposome compositions listed in Tab. 2.1 and 2.2 were tested.

\subsubsection{Applying liposome to side view preparation}

The v3V explant was prepared as described in Sec. 2.4.1. Then, the tissue of the v3V was cut further into thin slices along the anterior-posterior axis. A silicone spacer was placed on a $24 \times 50 \mathrm{~mm}$ cover slip (thickness No. 1) and 300 $\mu \mathrm{L}$ DMEM added inside the chamber. The v3V slices were transferred to the chamber. The slice was turned, so that the ciliary beating could be observed from the side. A smaller cover glass was placed on top. Ciliary beating was visualized and recorded by differential interference contrast (DIC) microscopy. Afterwards fluorescently-labeled liposomes diluted in DMEM (D1145, Sigma Aldrich, USA) in a ratio of 1:400 were added. Ciliary beating and fluorescent liposomes were imaged simultaneously over time.

A Leica DMI6000 microscope with CTR6500 and an Orca flash 4.0 camera (HAMA$M A T S U$ ) was used for imaging. The microscope was also equipped with DIC optics. The objectives 20x (HC PL APO 20x/0.70 dry) and 40x (HC PL FLUOTARL 40x/0.60 dry) were used for imaging.

These experiments were performed in the lab of Dr. Cecilia Lo of the University of Pittsburgh.

\subsubsection{Incubation of ventral third ventricle explant in liposome solution}

Prior to the experiment, 4-well cell culture plates were filled with $2 \%$ agarose (Biozym LE Agarose, Biozym Scientific GmbH, Germany) to form a $5 \mathrm{~mm}$ thick film covering the 


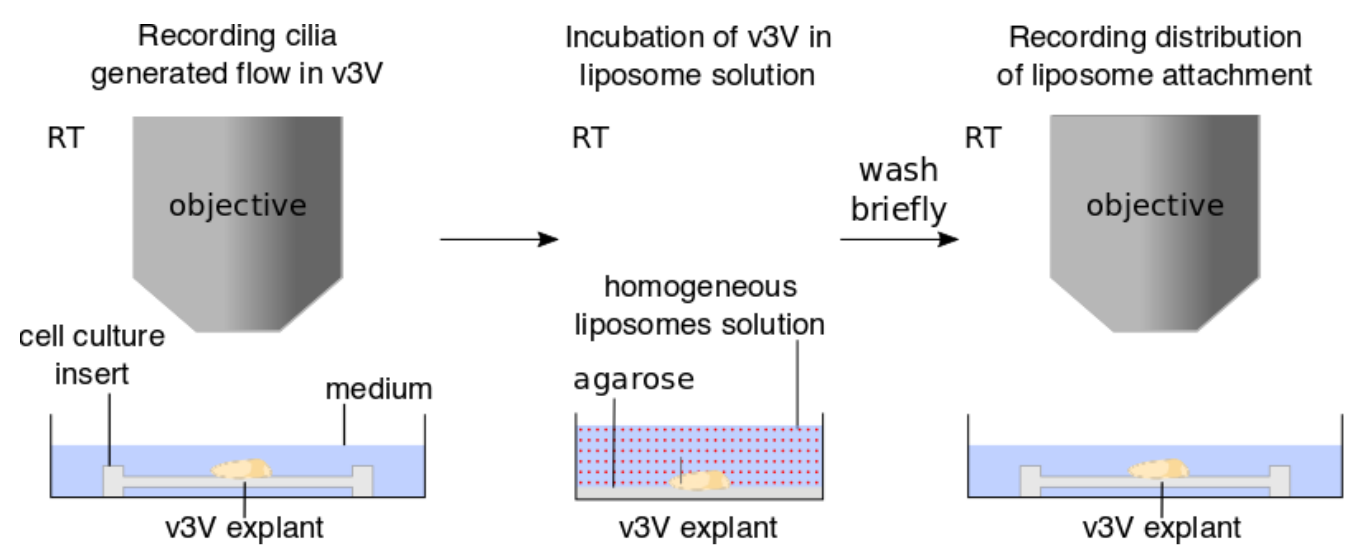

Figure 2.2: Experimental schema of v3V tissue explant incubation in liposome solution. The freshly dissected tissue explant was placed on top of a cell culture insert in a petri dish containing DMEM and fluorescent beads. The cilia-generated near wall flow was recorded. Afterwards, a homogeneous diluted solution of fluorescently-labeled liposomes was prepared and filled into prior prepared well of a cell culture plate with a thin layer of agarose at the bottom. The v3V tissue explant was transferred to the filled well and pinned down on the agarose film using an insect pin. Then the tissue explant was incubated at RT. After the chosen incubation time the tissue explant was washed briefly with DMEM and transferred back to the petri dish. The ependymal wall was not touched during the transfers and the ependymal cilia were always facing upwards. Then the whole area of the $\mathrm{v} 3 \mathrm{~V}$ was recorded again.

bottom of each well. Fluorescently-labeled liposomes were prepared as described in Sec. 2.3.2.

The v3V was freshly prepared and the cilia-generated flow recorded as described in Sec. 2.4.1 and 2.4.2. Fluorescently-labeled liposomes were diluted in DMEM to a total volume of $500 \mu \mathrm{L}$ per tissue explant. The dilution ratio varied from 1:10 to 1:500. For each tissue explant a well of a prepared cell culture plate was filled with $500 \mu \mathrm{L}$ diluted liposome solution. Each explant was transferred to a separated well with the v3V ependymal wall facing upwards. The tissue was pinned down on the agarose film using Austerlitz insect pins (12 mm length x $0.10 \mathrm{~mm}$ diameter, minutiens in stainless steel). The ependymal wall was not touched during transfer and pins were placed in adjacent brain parenchyma only (see Fig. 2.2).

The v3V explant was incubated in the diluted liposome solution for $10 \mathrm{~min}$ up to $1 \mathrm{~h}$ at room temperature. After incubation, the v3V explant was washed briefly with DMEM. The insect pin was removed and $\mathrm{v} 3 \mathrm{~V}$ explant transferred back to the prior used petri dish.

Then, the whole $\mathrm{v} 3 \mathrm{~V}$ ependymal wall was imaged by recording short movies in a way that each area was in focus. Each area was recorded with two excitation wavelengths. 
The multi-fluorescent beads were visible at both wavelengths, while fluorescently-labeled liposomes were only visible at one wavelength. This allowed for later differentiation between signals caused by fluorescent liposomes and fluorescent beads stuck on ependymal cells.

All imaging was performed using a Leica DMR upright microscope with Mercuryvapour-lamp and tubus factor 1.25. Cilia-generated flow was recorded at 5x magnification and liposome attachment sites were recorded at either 5x or 10x magnification.

\section{Kinetics and influence of liposome attachment}

The fluorescently-labeled liposomes were diluted with DMEM at a ratio of 1:10, 1:250 and 1:500. The cilia-generated near wall flow was recorded and the v3V explant transferred to a prepared cell culture plate as described above. For each dilution ratio, a v3V explant was incubated in homogeneous liposome solution for $10 \mathrm{~min}, 30 \mathrm{~min}$ or $1 \mathrm{~h}$. Next the $\mathrm{v} 3 \mathrm{~V}$ explant was washed briefly with DMEM, transferred back to former used petri dish and ciliary near wall flow was recorded again. Afterwards the whole v3V ependymal wall was recorded as described above with 10x magnification. Additionally, the inflow region, the separatrix region, the region of flow 6 and the outflow region were imaged at 20x (HC PL Fluostar 20x/0.50 PH2) magnification (see Fig. 1.1). The cilia-generated near wall flow was then recorded again.

\section{Mapping of liposome attachment site}

All recorded movies of the ventral third ventricle wall after incubation in homogeneous fluorescently-labeled liposomes solution were analyzed using Fiji [78]. Each movie was imported as image sequence and projected using Sum Slices. Every area of the v3V ependymal wall was imaged with two excitation wavelengths. The projection images of associated areas were merged and the resulting image converted to RGB colors. Contrast and brightness were adjusted and the merged image saved as .png and tiff format.

The merged images were stitched together by hand using Apache OpenOffice version 4.1.5. The multi-fluorescent beads, which were stuck on the apical ependymal cell surface, were used as fiducials for accurate overlapping of neighboring images. In oder to ensure that only ependymal surface in focus contribued to the resulting map of the $\mathrm{v} 3 \mathrm{~V}$ ependymal wall, the images were cropped. 


\subsubsection{Live imaging of liposome-labeled cilia}

Ventral third ventricle open pouch preparation was incubated in homogenous fluorescentlyliposome solution diluted with DMEM (D1145, Sigma Aldrich, USA) in a ratio of 1:400. A silicone spacer was placed on a cover glass of thickness No. 1 and size 24x50 mm, forming a chamber. $300 \mu \mathrm{L}$ DMEM were filled into the chamber on the cover glass. Tissue explant was transferred to the chamber with the ependymal wall facing downwards. A smaller cover glass of the same thickness was positioned on top.

The sample was placed in a Leica DMi8-CS inverted microscope equipped with a TCS SP8 confocal system. The hardware was operated by Leica LAS X software. Ciliary beating was imaged at 20x (HC PL APO CS2 20x/0.75 IMM) magnification in xyt-mode or xyzt-mode with $117 \mathrm{fps}$. The movies were analyzed using the Multi Kymograph plugin of Fiji [78].

These experiments were performed in the lab of Dr. Cecilia Lo of the University of Pittsburgh.

\subsubsection{Staining of ventral third ventricle}

Following the injection of fluorescently-labeled liposomes on v3V explant or incubation of explants in homogeneous liposome solution, the tissue was fixed with 4\% PFA (paraformaldehyde, Sigma Aldrich,Germany) for $30 \mathrm{Min}$ on ice and $30 \mathrm{Min}$ at room temperature. In case of mCLING-labeled liposomes $4 \%$ PFA with $0.2 \%$ gluteralaldehyde was used for fixation as recommended by H. N. Revelo et al. [85]. Then the tissue was washed with phosphate-buffered saline (PBS, $150 \mathrm{mM}$ sodium chloride and $20 \mathrm{mM}$ disodium-hydrogen-phosphate with $\mathrm{pH}=7.4$ ) for $5 \mathrm{~min}$.

A 0.5-millimeter-thick silicone spacer (made by the Institute workshop) was glued on a slide with Pritt Original glue stick (Henkel AG\&Co, Germany). VECTASHIELD HardSet antifade mounting media with DAPI (H-1500) and with TRITC-phalloidin (H-1600), to visualize nuclei and F-actin, were mixed in a ratio of 1:1. A drop of mounting medium mixture was filled between the spacer's walls and the fixed tissue positioned inside with the ependymal wall facing upwards. Finally, a round cover glass of thickness No. 1 was placed on top of the spacer and sealed with nail polish. The sample was stored at $4{ }^{\circ} \mathrm{C}$ until imaging.

The fixed v3V samples were imaged using a OLYMPUS IX81 microscope equipped with a FluoView FV1000 confocal laser scanning system. The setup included a $405 \mathrm{~nm}$, 
$458 \mathrm{~nm}, 488 \mathrm{~nm}, 515 \mathrm{~nm}, 559 \mathrm{~nm}$ and $635 \mathrm{~nm}$ laser module. All hardware was operated by OLYMPUS FV10-ASW Version 4.2 software. Image stacks were taken with 10x (UPLFL 10x 0.30), 40x (U PLSAPO 40x/0.90) or 60x (U PLSAPO 60x O 1.35) magnification in sequential frame mode.

\subsection{Interaction of extracellular vesicles with ventral third ventricle ependymal cells}

\subsubsection{Incubation of ventral third ventricle in extracellular vesicle solution}

A fresh v3V explant was prepared as described in Sec. 2.4.1 and the cilia-generated bead flow recorded as described in Sec. 2.4.2. As explained in Sec. 2.6.3 for fluorescent liposomes, v3V explant was transferred to a prior prepared 4-well plate and incubated in fluorescently-labeled EVs solution diluted in DMEM. The explant was washed with DMEM after incubation and transferred back to the former used petri dish. The ciliadriven bead flow was recorded again and the whole ependymal wall of the v3V was imaged. Afterwards, the cilia-generated bead flow was recorded every few hours until $21 \mathrm{~h}$ after incubation.

\subsubsection{Applying extracellular vesicle to side view preparation}

The v3V explant was prepared as described in Sec. 2.4.1 and further cut into thin slices along the anterior-posterior axis. The $\mathrm{v} 3 \mathrm{~V}$ slices were transferred to a petri dish with a glass bottom and filled with DMEM (D1145, Sigma USA). Ciliary beating was observed by differential interference contrast microscopy and short movies were recorded. Fluorescently-labeled extracellular vesicles were added to the volume using a pipet or applied close to the tissue using a FemtoJet 4i (Eppendorf, USA) with self-made glass capillaries. The capillaries were made from standard borosilicate tubing (length $15 \mathrm{~cm}$ and outer diameter $1 \mathrm{~mm}$ ) using a micropipette puller Model P-1000 from Sutter Instruments. The transport of fluorescently-labeled EVs was observed simultaneously with the beating of ependymal cilia and short movies were recorded.

The imaging was done using a Leica DMI6000 microscope with CTR6500 and an Orca flash 4.0 camera from HAMAMATSU. The microscope was equipped with DIC optics. The 20x and 40x objectives were used for imaging. 
The experiment was performed in the lab of Dr. Cecilia Lo of the University of Pittsburgh.

\subsection{Liposome interaction with diverse cell types}

The interaction between liposomes and ciliated ependymal cells was studied on mouse trachea cells, oviduct tissue, sperm cells, as well as rabbit oviduct and human nasal epithelia cells. Except for liposome interaction study on rabbit oviduct all experiments were performed in the lab of Dr. Cecilia Lo of the University of Pittsburgh.

\subsubsection{Mouse trachea tissue and cells}

Post mortem the mouse neck skin was opened with two cuts without damaging any blood vessels. Trachea was exposed and cleaned from excess tissue using tweezers. Trachea was cut at the inferior end behind the tweezers. Then a second cut was made $1 \mathrm{~cm}$ superior from the holding tweezers. The isolated trachea was transferred to a petri dish filled with DMEM (D1145, Sigma Aldrich, USA). Thin rings were cut from the isolated trachea and opened up to stripes.

The trachea stripes were transferred to a drop DMEM on a $24 \times 50 \mathrm{~mm}$ cover glass surrounded by a silicone spacer. A smaller cover glass was put on top of the spacer. The ciliary beating was recorded using DIC microscopy. Afterwards, the DMEM was exchanged by fluorescent liposomes diluted 1:400 in DMEM. The interaction between cilia and liposomes was recoded over time.

Furthermore, ciliated trachea cells were scratched from freshly prepared trachea stripes and transferred to a DMEM filled 4-well plate with a round cover glass on the bottom. The cells were kept at $37^{\circ} \mathrm{C}$ until they set to the cover glass. A chamber was prepared by placing a silicon spacer on a $24 \times 50 \mathrm{~mm}$ cover glass and adding $200 \mu \mathrm{L}$ of fluorescent liposome solution diluted 1:400 in DMEM.

The trachea tissue was imaged using a Leica DMI6000 with CTR6500, which was equipped with an Orca flash 4.0 camera from HAMAMATSU and DIC optics. The imaging was performed at 20x and 40x magnification. Additionally, the trachea cells were imaged using a Leica DMI8-CS inverted microscope equipped with a TCS SP8 confocal system at 20x (HC PL APO CS2 20x/0.75) magnification. 


\subsubsection{Mouse oviduct}

Mouse oviduct was isolated directly after sacrifice and transferred to a petri dish filled with DMEM (D1145, Sigma Aldrich, USA). Small slices of the oviduct were cut by using sharpened tungsten needles. A silicone spacer was put on a $24 \times 50 \mathrm{~mm}$ cover glass. $300 \mu \mathrm{L}$ DMEM were added on top of the cover glass and oviduct slices were carefully transferred onto the cover glass. A smaller cover glass was placed on top of the spacer. After imaging the ciliary beating using DIC, fluorescently-labeled liposomes diluted in DMEM (1:300) were added. Afterwards the ciliary beating and fluorescent liposomes were recorded simultaneously over time at 20x and 40x magnification.

The microscopy was performed using a Leica DMI6000 microscope with CTR6500 and an Orca flash 4.0 camera (HAMAMATSU) equipped with DIC optics.

\subsubsection{Rabbit oviduct}

The rabbit tissue was generously provided by Dr. Claudia Richter of the MPI for Dynamics and Self-Organization. The ovary and oviduct of a rabbit was isolated after the death and transferred to a falcon tube filled with DMEM for washing. Excess and fat tissue were removed using tweezers. The oviduct was cut into pieces starting from the ovary side by using fine spring scissors. The oviduct pieces were transferred to a new petri dish with glass bottom and filled with fresh DMEM (21063, ThermoFisher Scientific, Germany). The ciliary beating was observed by DIC microscopy. The fluorescentlylabeled liposomes were added with a pipette and the distribution recorded using fluorescent microscopy. The interaction between fluorescently-labeled liposomes and cilia of the oviduct was imaged over time.

The microscopy was done using an upright Leica DMR microscope with mercuryvapour-lamp and a Cascade II camera Photometrics. The microscope was equipped with DIC optics and the camera operated by MultiRecorder software (Johannes SchröderSchetlig, MPI for Dynamics and Self-Organization, Göttingen). All imaging was done at $5 \mathrm{x}, 10 \mathrm{x}$, or $60 \mathrm{x}$ magnification.

\subsubsection{Mouse sperm cells}

Mouse testicles were isolated directly after sacrifice and transferred to a petri dish filled with DMEM (D1145, Sigma Aldrich, USA). Next, holes were punched into the testicles using tweezers. Then, the testicles were incubated at $37^{\circ} \mathrm{C}$ for at least $10 \mathrm{~min}$. 
A $24 \times 50 \mathrm{~mm}$ cover slide of thickness No. 1 was prepared by placing a narrow strip of tape on each long edge. A smaller cover glass of the same thickness was placed on top of the spacers. Fluorescently-labeled liposomes were diluted in DMEM in a ratio of 1:500. $200 \mu \mathrm{L}$ of diluted liposome solution were mixed with $50 \mu \mathrm{L}$ of sperm cell solution and added between the two cover slides. The sperm cells and fluorescent liposomes were simultaneously imaged using DIC and fluorescent microscopy. The interaction between liposomes and sperm cells was observed over time from directly after adding fluorescent liposomes to $1.5 \mathrm{~h}$ after the addition.

The imaging was performed using a Leica DMI6000 microscope with CTR6500 and an Orca flash 4.0 camera (HAMAMATSU). The microscope was equipped with DIC optics. The 20x, 40x and 60x objectives were used for imaging.

\subsubsection{Human nasal epithelia cells}

Human nasal epithelia cells were scratched from a healthy volunteer and transferred to a $15 \mathrm{~mL}$ falcon tube filled with Leibovitz's buffer. The sample was spun down at $1.2 \times 1000$ rpm for $6 \mathrm{~min}$. The supernatant was aspirated down to $1 \mathrm{~mL}$ and suspended in $1 \mathrm{~mL}$ of supernatant. Then the sample was transferred to a $1.5 \mathrm{~mL}$ epp tube spun down again at $3.2 \times 1000 \mathrm{rpm}$ for $3 \mathrm{~min}$. The supernatant was aspirated down to $300 \mu \mathrm{L}$. A tissue homogenizer was used to break up the sample with three quick bursts of $1 \mathrm{~s}$ each. $40 \mu \mathrm{L}$ of the remaining sample was pipetted on a polylysine coted round coverslip and spread out carefully.

Fluorescently-labeled liposomes were diluted in 1:400 in DMEM. $200 \mu \mathrm{L}$ were added on top of a $24 \times 55 \mathrm{~mm}$ cover glass with silicone spacer. A coverslip with attached human nasal epithelia cells was placed on top of the space with the cells facing into the liposome solution. Fluorescent liposomes and ciliated epithelia cells were imaged using a Leica DMI8-CS inverted microscope equipped with a TCS SP8 confocal system at 20x magnification.

\subsection{Experimental workflow}

Figure 2.3 and 2.4 show a summary of the experimental approach to analyze liposome and extracellular vesicle transport and interaction with the ciliary logistic network and ependymal cells of the $\mathrm{v} 3 \mathrm{~V}$. 


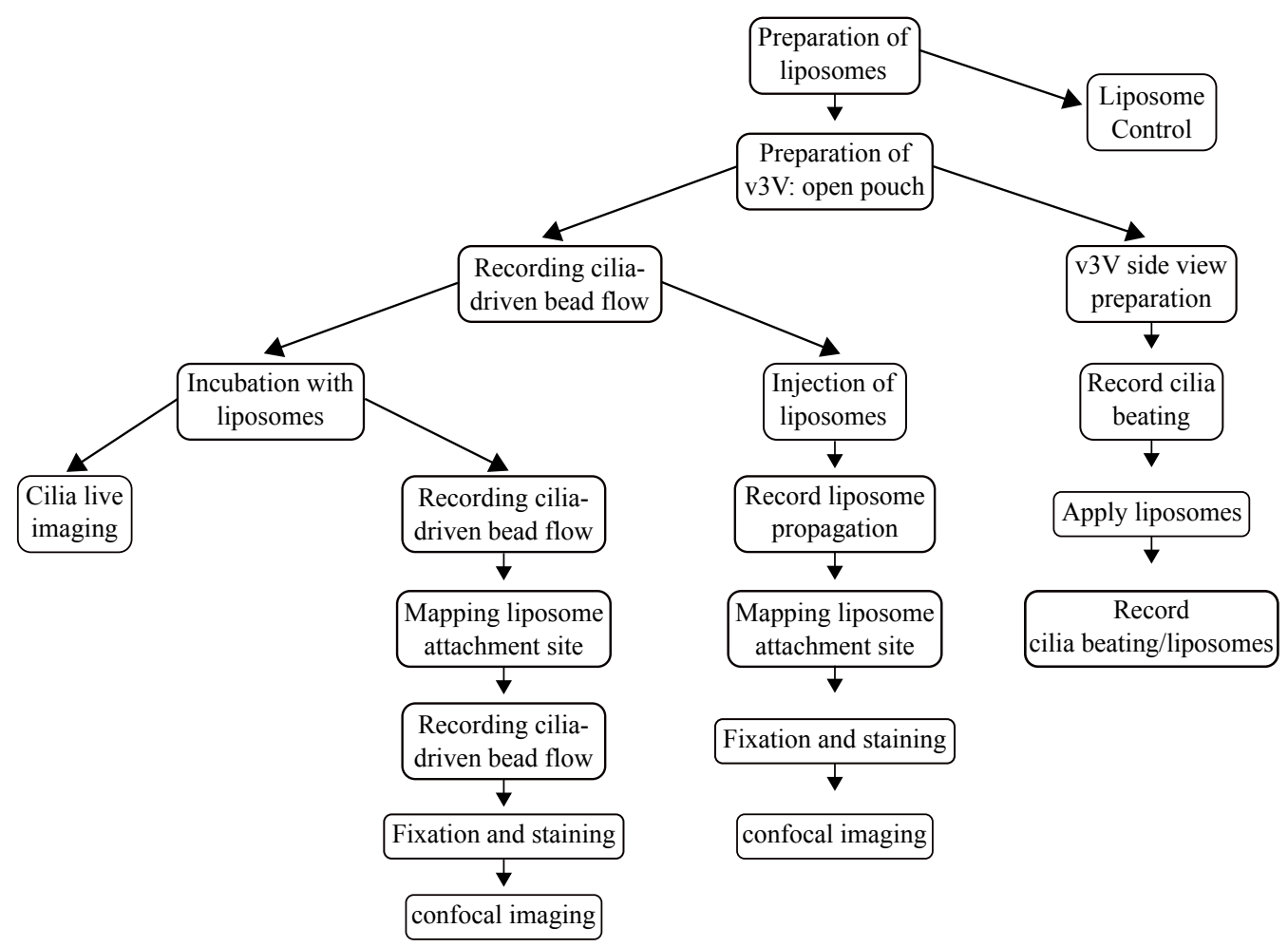

Figure 2.3: Experimental workflow to analyze the interaction between v $3 \mathrm{~V}$ cilia and fluorescentlylabeled liposome.

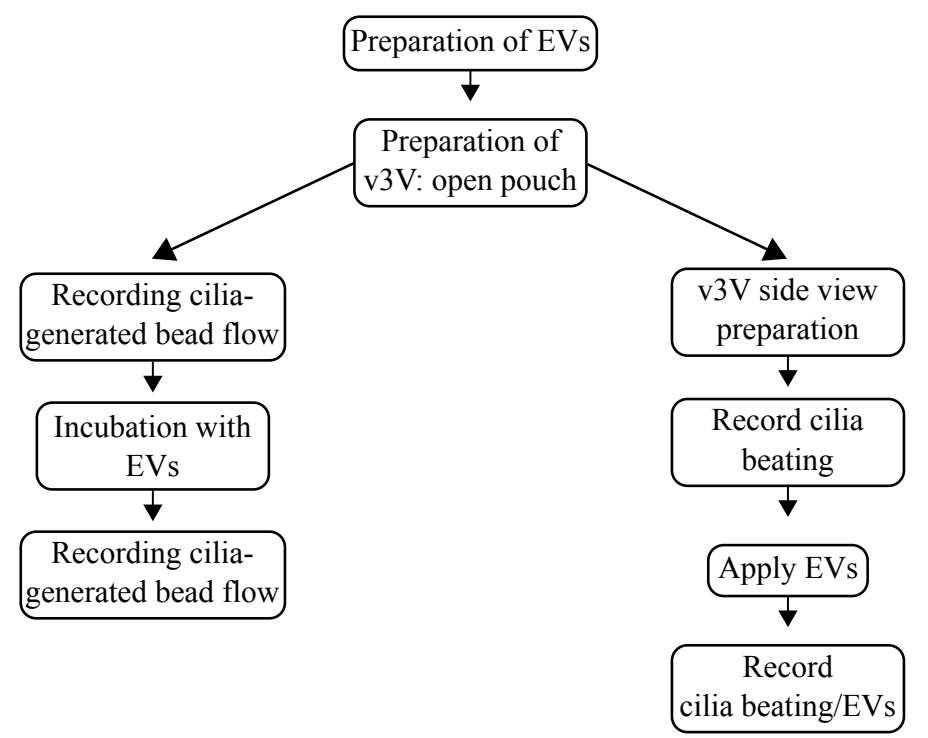

Figure 2.4: Experimental workflow to analyze fluorescently-labeled extracellular vesicle interaction with v3V ciliary logistic network and ependymal cells. 


\section{Chapter 3}

\section{Results}

\subsection{Characterization of liposome transport via the cilia logistic network}

\subsubsection{Analysis of liposome properties}

To accurately study the transport of potential cargo packaged in vesicles by the ciliary logistic network, a detailed characterization of cargo-specific transport properties is required. These properties include size, concentration and surface-charge. For transport across long distances, packaging of cargo into extracellular vesicles, such as exosomes and ectosomes, may provide stability and site-directed delivery. Transport and delivery of cargo mostly depends on vesicle properties. To study transport of such extracellular vesicles in the $\mathrm{v} 3 \mathrm{~V}$, fluorescently-labeled lipid vesicles were artificially produced. These so-called liposomes were produced that had diverse surface properties. This allowed to study the effect on vesicle transportation and interaction with potential target cells.

\section{Liposome size distribution}

Liposomes of different lipid compositions were prepared by extrusion and size exclusion chromatography aiming for different size distributions, which correspond to the size range of natural exosomes $(30 \mathrm{~nm}$ to $150 \mathrm{~nm}$ [4]). Nanoparticle tracking analysis (NTA) and electron microscopy (EM) were used to measure size distributions.

Nanoparticle tracking analysis allows the measurement of the size distribution of particles in a given solution by visualizing them via dynamic light scattering and tracking their Brownian motion $[86,87]$. The mean square displacement of each tracked particle is 
used to calculate the hydrodynamic diameter using the Stokes-Einstein equation [86, 87]. $\mathbf{A}$ and $\mathbf{B}$ in Figure 3.1 show representative NTA measurements of liposomes prepared by extrusion. Both size distributions were measured from a 1:2000 diluted liposome solution, diluted in HP-150 buffer, and averaged over five single measurements per sample. The size distribution of liposomes prepared from composition VIII (Tab. 2.2, Fig. 3.1 A) shows a sharp peak at $82 \mathrm{~nm}$ and $90 \%$ of the measured particles are smaller or equal to $114 \mathrm{~nm}$. Liposomes of composition IX (Tab. 2.2, Fig. 3.1 B) show a sharp peak at $78 \mathrm{~nm}$ in their size distribution and $90 \%$ of the measured particles are smaller or equal to $111 \mathrm{~nm}$. Hence, the preparation of liposomes by extrusion resulted in very similar size distributions for both lipid compositions.

Figure 3.1 C shows a representative size distribution of liposomes (composition II, Tab. 2.1) prepared by size exclusion chromatography, measured from a 1:500 diluted liposome solution, diluted in HP-150 buffer. The size distribution shows a main peak at $65 \mathrm{~nm}$, with two smaller peaks around $25 \mathrm{~nm}$ and $89 \mathrm{~nm} .90 \%$ of the measured particles are smaller or equal to $98 \mathrm{~nm}$. NTAs lower detection limit of small particles is limited by the refraction index of the given particles, which depends on the material and increases with the size of the particle. Due to this restriction, NTAs theoretical detection limit of liposomes are particles in the size range of $55 \mathrm{~nm}$ to $60 \mathrm{~nm}$ [88].

Electron microscopy images of liposome samples prepared by size exclusion chromatography revealed a large number of small particles mixed with a few larger size lipsome clusters (Fig. 3.1 D). Several EM images per liposome sample were used to calculate the size distribution of the sample (cf. Fig. 3.1 E). Only single particles were measured and clusters were excluded from the distribution. The liposome diameter mainly varies from $20 \mathrm{~nm}$ to $45 \mathrm{~nm}$ with a maximum around $30 \mathrm{~nm}$. This result was similar for $n=6$ analyzed liposome samples prepared by size exclusion chromatography.

Apparently, the NTA measurement of liposomes prepared by size exclusion chromatography seems to overrepresent liposome clusters at the expense of single particles. Although, liposomes also can be morphologically affected by the embedding for EM [89]. The shape of liposomes in the analyzed samples appeared normal. Thus, the measured size distribution of size exclusion chromatography prepared liposomes from EM images seems to be more reliable.

Consequently, the size distribution of small liposomes prepared by size exclusion chromatography was determined by EM, while larger liposome prepared by extrusion were analyzed by NTA. 

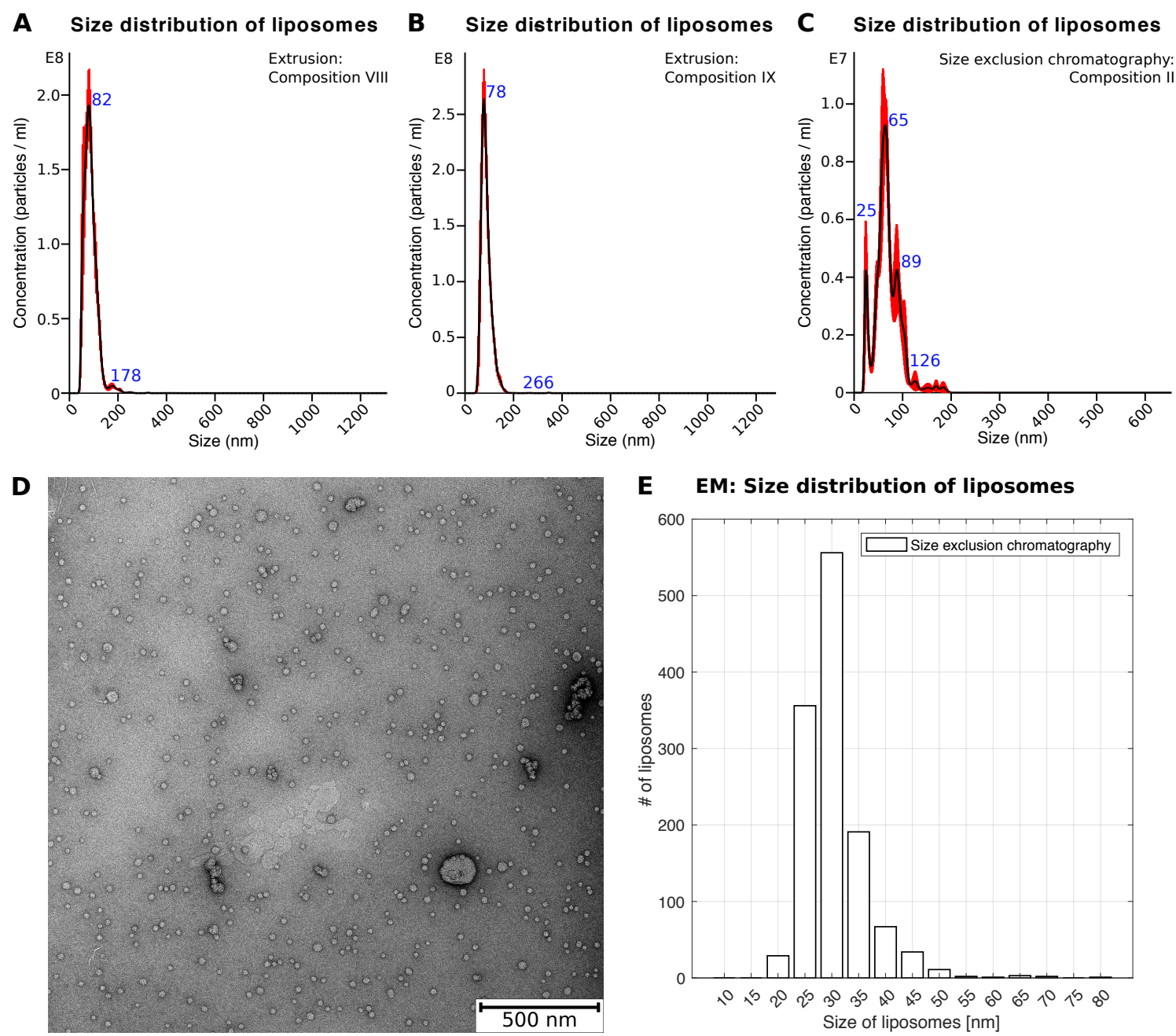

Figure 3.1: Liposomes prepared by size exclusion chromatography and extrusion were characterized by electron microscopy (EM) and nano-particle tracking (NTA). A to $\mathbf{C}$ show representative size distributions of liposomes measured by NTA. A Liposomes of composition VIII (Tab. 2.2) were prepared by extrusion. The size distribution has a sharp peak at $82 \mathrm{~nm}$. B Liposomes of composition IX (Tab. 2.2) were prepared by extrusion. The size distribution shows a sharp peak at $78 \mathrm{~nm}$. Although different lipid compositions were used, extrusion produced identical size distributions. C Liposomes of composition II (Tab. 2.1) were prepared by size exclusion chromatography. The size distribution shows multiple peaks $25 \mathrm{~nm}, 65 \mathrm{~nm}$ and $89 \mathrm{~nm}$. D presents a EM image of liposomes prepared by size exclusion chromatography. Small single particles make up the majority of the particles. In addition, few larger clusters of liposomes are present. E Representative size distribution of liposomes prepared by size exclusion chromatography and measured from EM images. The size of single particles was measured from several images per sample. Clusters of liposomes were not included in $\mathbf{E}$. 


\subsubsection{Propagation of liposomes in cilia-generated near wall flow}

The v3V cilia-generated flow network and its potential for transporting CSF cargo along the ependymal walls was previously described and characterized by R. Faubel et al. $[2,81]$. The cilia-driven near wall flow was analyzed by tracing $1 \mu \mathrm{m}$ fluorescent beads, revealing a highly complex flow pattern throughout the v3V (Fig. 1.1), which matches the underlying cilia beating direction. Locally applied fluorescein isothiocyanate (FITC)dextran $(70 \mathrm{kDa})$ was distributed along prescribed streamlines by the flow map [2]. These results led to the hypotheses, that $\mathrm{v} 3 \mathrm{~V}$ flow maps may reflect the propagation of macromolecules in the CSF [2]. Additionally, the cilia-generated flow of the v3V forms boundaries and thereby separates the v3V's volume [2]. As a result of this separation, substances present in the CSF are only able to reach specific locations [2].

Transport of biologically relevant cargo in this flow network was probed by releasing fluorescently-labeled liposomes of defined size and composition at specific locations of the wall of v3V. All experiments were performed at room temperature (RT).

Fluorescently-labeled liposomes of composition I (Tab. 2.1) were prepared by size exclusion chromatography. Applied at the head of flow 1 and 2 liposomes did fan out, distributing over a large area from the inflow ventrally towards the base of the v3V and even further along flow 8 (Fig. $3.2 \mathrm{C}$ and $3.3 \mathbf{C}$ ). Applied at the head of flow 3 the liposomes were transported posteriorly towards the middle of the $\mathrm{v} 3 \mathrm{~V}$ along a thin streamline (Fig. 3.2 D, E and 3.3 D). Upon reaching the separatrix, directly in the area of opposing flow 3 and flow 4, the liposomes left the near wall flow and were transported away from the ependymal surface (Fig. 3.2 D). When transported along flow 3 and flow 5 liposomes reached flow 6 and the base of the v3V (Fig. 3.2 E and Fig. 3.3 D).

Liposomes applied at the head of flow 4 were transported anteriorly towards the separatrix and bent downwards when reaching the opposing flow 3 (Fig. $3.2 \mathbf{F}$ and $3.3 \mathbf{E}$, F). Even upon applying a large amount of liposome solution, liposomes did not reach the region located anterior of the separatrix (Fig. 3.3 F). Liposomes injected into the near wall flow 6 close to the dorsal tissue border of the $\mathrm{v} 3 \mathrm{~V}$ or close to the tanycyte region were transported ventrally towards the base of the ventricle (Fig. $3.2 \mathbf{G}$ and $3.3 \mathbf{F}, \mathbf{G}$ ). Applied in flow 7 starting at the dorsal tissue border or close to the tanycyte region, liposomes were transported towards the posterior duct (Fig. 3.2 $\mathbf{H}$ and 3.3 H). Altogether, fluorescent liposomes are transported along the same pathways of the $\mathrm{v} 3 \mathrm{~V}$ cilia-generated flow network as fluorescent beads and FITC dextran. This finding suggests that the pro- 
pagation pattern of vesicles-encapsulated CSF-components in the $\mathrm{v} 3 \mathrm{~V}$ is not changed by packaging them into small extracellular vesicles.
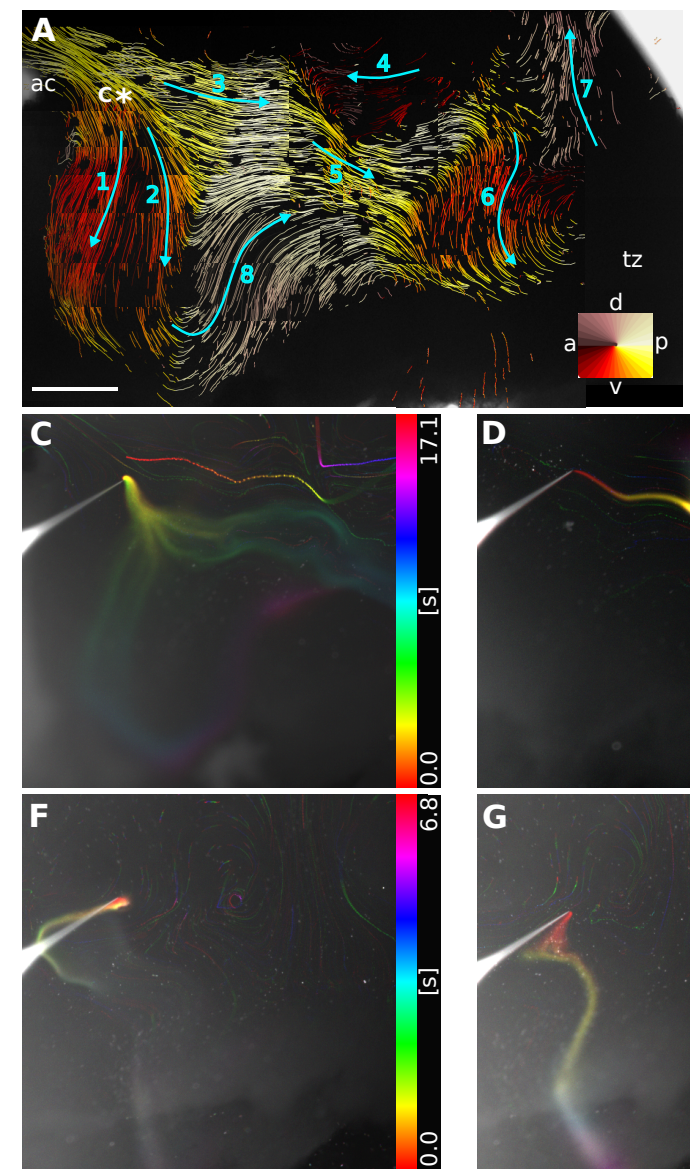
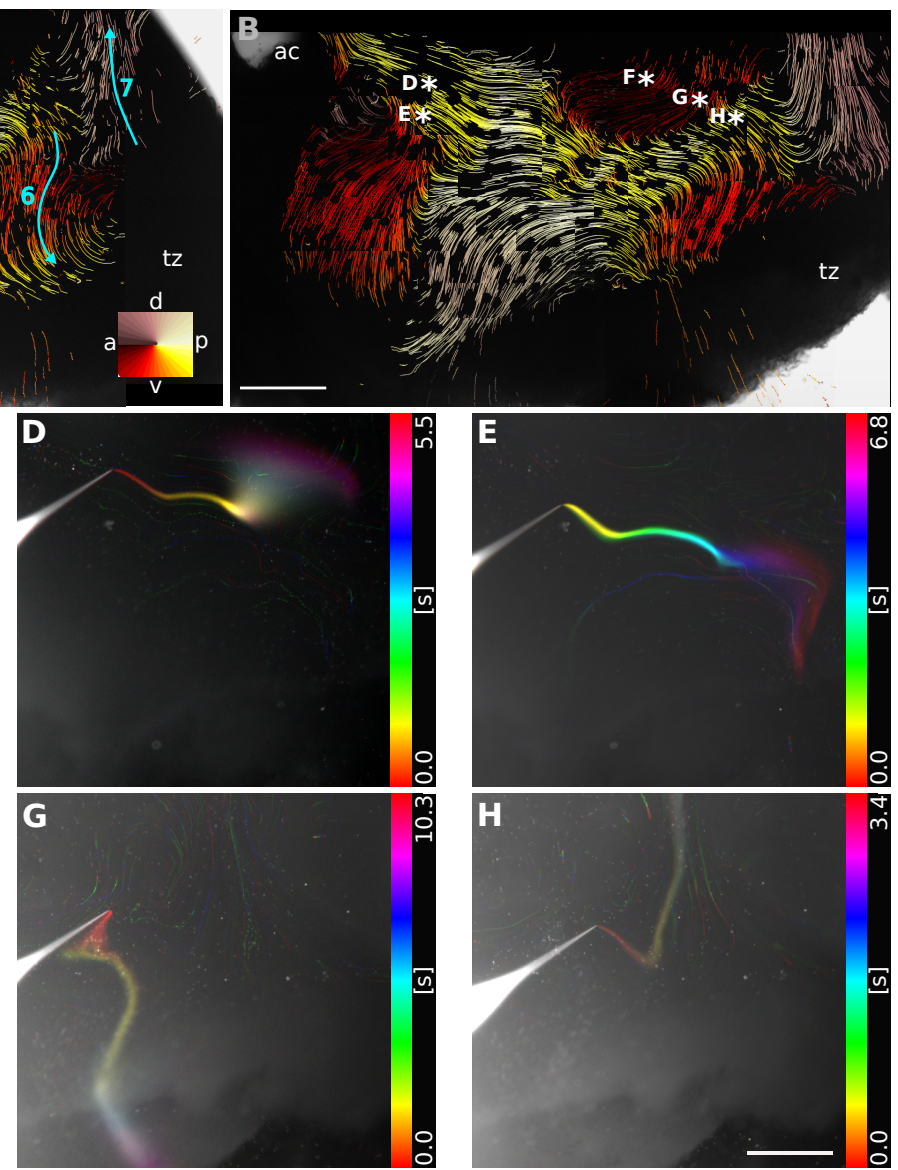

Figure 3.2: The propagation of fluorescent liposomes of composition I (Tab. 2.1) was studied by injecting small amounts into the ciliary near wall flow of the v3V. A and $\mathbf{B}$ show representative flow maps of the v3V from the right hemisphere at ZT10. The legend in A shows the flow direction color code, with $\mathrm{d}=$ dorsal, $\mathrm{p}=$ posterior, $\mathrm{v}=$ ventral and $\mathrm{a}=$ anterior. The light blue arrows indicate the flow direction and numbering of each flow. The light blue $\mathrm{S}$ indicates the position of the separatrix. The white stars represent the injection site shown in $\mathbf{C}$ to $\mathbf{H}$. Images shown in $\mathbf{C}$ to $\mathbf{H}$ are projections of liposomes pathways in the cilia near wall flow with respect to different injection sites. A temporal color code was applied to all projection using Fiji [78]. The temporal color code and time scales is shown next to each image. $\mathbf{C}$ liposomes were injected in the inflow region and are distributed along flow 1, 2, 8 and 3 to the posterior part of the v3V. D liposomes are transported along flow 3 and out of the focal plane upon reaching the separatrix. $\mathbf{E}$ liposomes are transported along flow 3 and 5 to the base of the v3V. F liposomes are transported along flow 4, bend downwards upon reaching the separatrix and are partly distributed out of the focal plane. G liposomes are transported along flow 6 towards the base of the v3V. $\mathbf{H}$ liposomes are transported along flow 7 towards the posterior duct. Scale bars $=300 \mu \mathrm{m}, \mathrm{ac}=$ anterior commissure, $\mathrm{tz}=$ tanycyte region, $\mathrm{s}=$ second. 

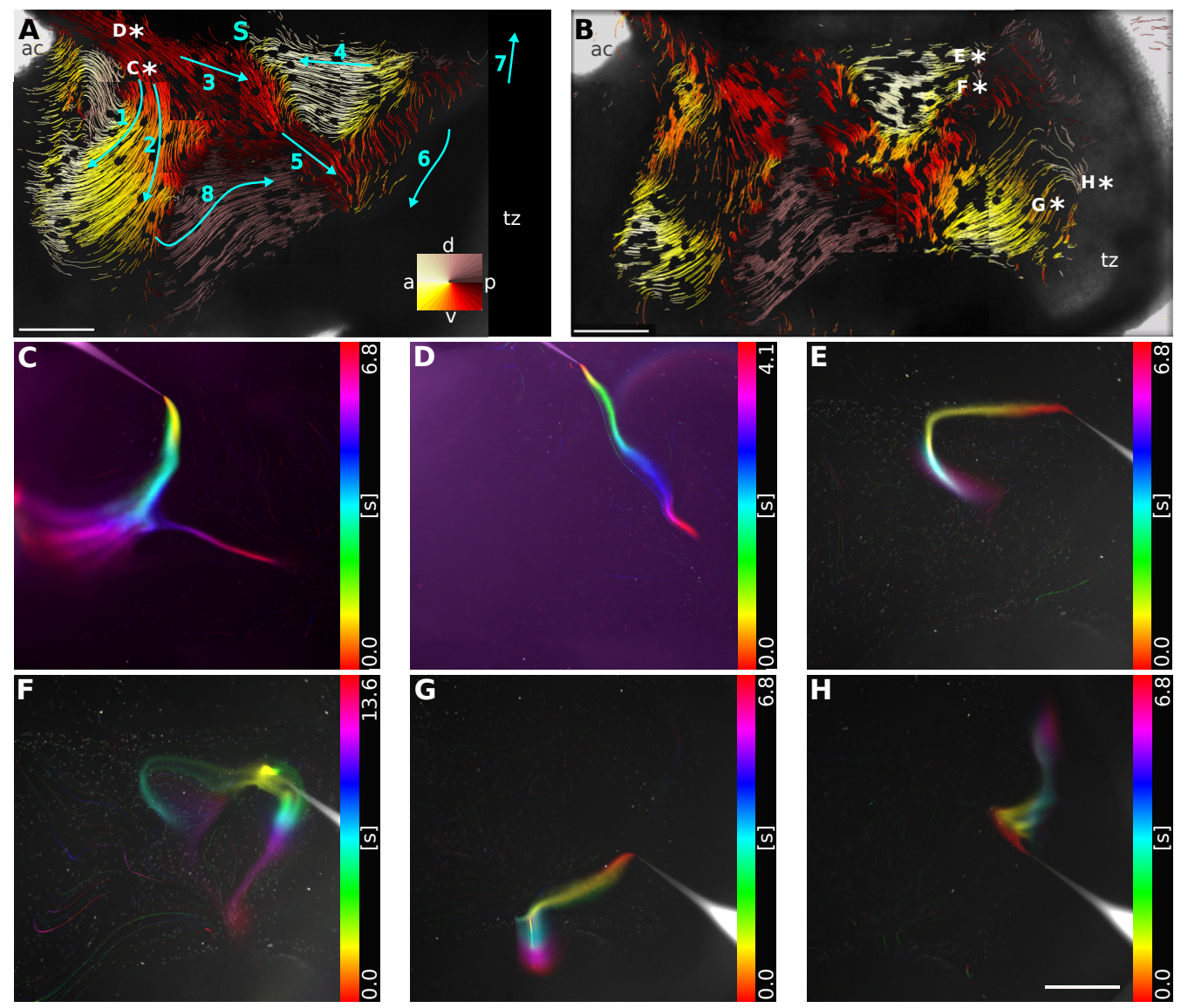

Figure 3.3: The propagation of fluorescent liposomes of composition I (Tab. 2.1) was studied by injecting small amounts into the ciliary near wall flow of the v3V. A and $\mathbf{B}$ show representative flow maps of the v3V from the left hemisphere at ZT10. The legend in $\mathbf{A}$ shows the flow direction color code, with $\mathrm{d}=$ dorsal, $\mathrm{p}=$ posterior, $\mathrm{v}=$ ventral and $\mathrm{a}=$ anterior. The light blue arrows indicate the flow direction and numbering of each flow. The light blue $\mathrm{S}$ indicates the position of the separatrix. The white stars represent the injection site shown in $\mathbf{C}$ to $\mathbf{H}$. Images shown in $\mathbf{C}$ to $\mathbf{H}$ are projections of liposomes pathways in the cilia near wall flow with respect to different injection sites. A temporal color code was applied to all projection using Fiji [78]. The temporal color code and time scales is shown next to each image. $\mathbf{C}$ liposomes were injected in the inflow region and fan out along flow 1,2 and 8. The image is rotated to the right in comparison to A. D liposomes are transported along flow 3 towards the separatrix. The image is rotated to the right in comparison to $\mathbf{A}$. $\mathbf{E}$ liposomes are transported along flow 4 and bent downwards upon reaching the separatrix. F liposomes are transported along flow 4 towards the separatrix and flow 6 towards the base of the v3V. G liposomes are transported along flow 6 to the base of the $\mathrm{v} 3 \mathrm{~V}$. $\mathbf{H}$ liposomes are transported along flow 7 towards the posterior duct. Scale bars $=300 \mu \mathrm{m}, \mathrm{ac}=$ anterior commissure, $\mathrm{tz}=$ tanycyte region, $\mathrm{s}=$ second. 


\subsubsection{Analysis of liposome propagation rates}

The propagation of fluorescently-labeled liposomes after application at different locations in the $\mathrm{v} 3 \mathrm{~V}$ was compared to the recorded bead-based flow maps of the cilia-generated near wall flow (Fig. 3.2 $\mathbf{A}$ and $\mathbf{B}$ with $\mathbf{C}$ to $\mathbf{H}$ and Fig. 3.3 $\mathbf{A}$ and $\mathbf{B}$ with $\mathbf{C}$ to $\mathbf{H}$ ). Liposomes transported in the near wall flow followed the prescribed streamlines as visualized by the flow map. Even over long distances, liposomes were transported precisely along the streamlines (Fig. 3.2 C). In the separatrix area, where flow 3 is opposed by flow 4, liposomes are transported up and away from the ependymal surface. Hence, the physical foreces that mediate this flow do not distinguish between $1 \mu \mathrm{m}$ latex beads and liposomes. Accordingly, extracellular vesicle-like cargo in the $\mathrm{v} 3 \mathrm{~V}$ is transported precisely along the cilia-prescribed streamlines.

The propagation velocity of liposomes along the streamlines of the near wall flow and the velocity of fluorescent beads was calculated using Fiji and Matlab. The velocities were added up from all v3V flows of $n=7$ mice (App. Tab. 5.2).

Following the application of liposomes into the near wall flow, the applied amount was transported and formed according to the cilia-driven flow. The propagation front of liposomes along the cilia-generated near wall flow was traced, the propagation distance measured and the velocity calculated. Individual liposomes, that may have been transported faster or attached to ependymal cells could not be traced and are therefore not included. The calculated propagation velocity of liposomes in the near wall flow varied from $120 \mu \mathrm{m} / \mathrm{s}$ to $300 \mu \mathrm{m} / \mathrm{s}$ with an average velocity of $(187 \pm 38) \mu \mathrm{m} / \mathrm{s}$ (Fig. $3.4 \mathrm{~A}$ ). Thus, the propagation of liposomes through the $\mathrm{v} 3 \mathrm{~V}$ takes only a few seconds.

The velocity of fluorescent beads transported along the cilia-generated flow was calculated with the same specimens and regions used for calculating the propagation velocity of liposomes. The velocity of beads was calculated using the 2D and 3D Particle Tracker of the Fiji plugin MOSAICsuite [83, 84] and Matlab. Unlike the applied liposomes, beads were tracked individually, including those that came into contact with the ependymal cells and were slowed down, or the ones that exited the near wall flow. This led to a wider range of velocities for the fluorescent beads than observed for the propagation of liposomes. The velocity of fluorescent beads transported in the cilia-driven flow varied from $10 \mu \mathrm{m} / \mathrm{s}$ to $550 \mu \mathrm{m} / \mathrm{s}$ with an average velocity around $(200 \pm 92) \mu \mathrm{m} / \mathrm{s}$ (Fig. 3.4 B).

Although, the range of measured velocities for liposome propagation and fluorescent beads differs, the mean velocities are similar and were represented most frequently in 
both distributions. The difference between the velocity range of liposome propagation and transported beads likely results from the calculation methods. Nevertheless, both velocities are typical for cilia driven flow [90]. It is likely that extracellular vesicles are transported within a similar velocity range at room temperature. The diameter of the ependymal cells apical side in the brains ventricular system is approximately $10 \mu \mathrm{m}$. Based on these observations, vesicles would be passing over this distance in 30 to $80 \mathrm{~ms}$.

Assuming that the cilia-generated near wall flow delivers CSF components, these results led to several thoughts of how extracellular vesicles could reach a target region. A target region for cargo transported by extracellular vesicles could be reached as EVs flow along their trajectories. Due to the transport velocity, the interaction time with the target region would be short. This suggests the involvement of additional mechanisms or cell structures to support an interaction. For example, microvilli protrude from the plasma membrane of ependymal cells in the $\mathrm{v} 3 \mathrm{~V}$, increasing the cell surface area and thus supporting adsorption [1]. Furthermore, the ependymal cell cilia could be involved, considering the direct contact of their membrane with the transported CSF components. Alternatively, it is possible that flows slow down at specific locations and EVs could bind to tissue.
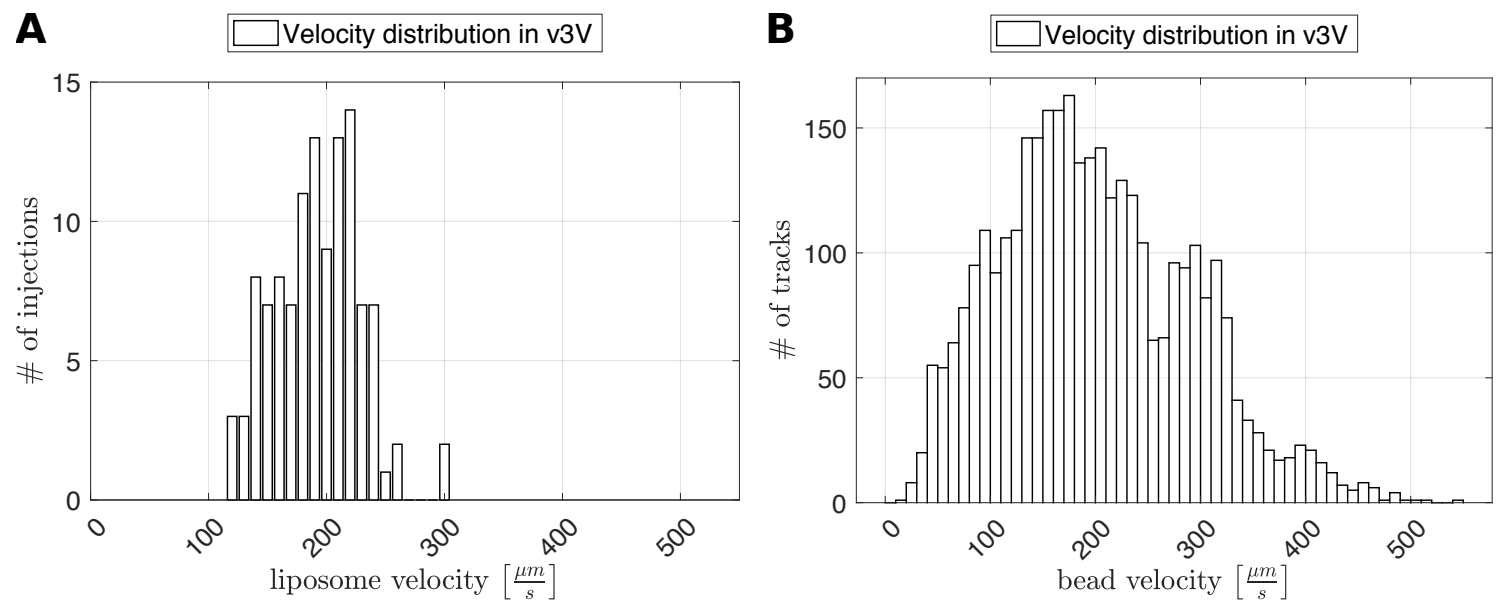

Figure 3.4: The velocity distribution of liposome propagation (A) and bead movement $(\mathbf{B})$ along the cilia near wall flow over the whole $\mathrm{v} 3 \mathrm{~V}$ was calculated from $n=7$ animals. The velocity of liposome propagation varied between $120 \mu \mathrm{m} / \mathrm{s}$ to $300 \mu \mathrm{m} / \mathrm{s}$ with an average value of $(187 \pm$ 38) $\mu \mathrm{m} / \mathrm{s}$. The bead velocity varied between $10 \mu \mathrm{m} / \mathrm{s}$ to $550 \mu \mathrm{m} / \mathrm{s}$ with an average value of $(200 \pm 92) \mu \mathrm{m} / \mathrm{s}$. 


\subsubsection{Transport of liposomes via the cilia network at Zeitgeber times 10h and}

23.5h

The cilia-generated flow in the $\mathrm{v} 3 \mathrm{~V}$ gives rise to a complex flow pattern, that reflects the beating direction of ependymal cilia [2]. It was demonstrated by Faubel et al. that the flow pattern in the $\mathrm{v} 3 \mathrm{~V}$ shows differences between ZT10, i.e., during the animals resting phase, and ZT23.5, i.e., during the animals active phase [2]. At ZT10 the separatrix, formed by the opposing flow 3 and flow 4, and flow 5 formed a intraventricular boundary [2]. At ZT23.5 a circular arrangement of cilia ventrally of the seperatrix gave rise to a circulating flow [2], called "whirl".

Fluorescent liposomes of composition I (Tab. 2.1), prepared by size exclusion chromatography, were applied to the cilia-generated near wall flow at ZT10 and ZT23.5. A concentrated liposomes solution applied at the head of flow 1 and flow 2 was fanned out and distributed within seconds along the cilia-prescribed streamlines similar at ZT10 and ZT23.5 (Fig. $3.3 \mathbf{C}$ and $3.5 \mathrm{~A}$ ). In accordance with the ZT10 flow pattern, liposomes locally applied to flow 3 , which were transported towards flow 5 , reached the base of the v3V (Fig. 3.2 E and 3.3 D). However, liposomes applied to flow 4, close to the separatrix, were transported and distributed ventrally along the near wall flow at ZT10 (Fig. 3.5 C), while in the presence of a whirl (ZT23.5), liposomes remained concentrated and were transported away from the ependymal cells (Fig. 3.5 B). This demonstrates the ability of cilia-driven flow to connect or separate volumes and thereby create subregions within the $\mathrm{v} 3 \mathrm{~V}$. It should be noted, that the separatrix region is located near the area, where the v3V ventricular walls come closest to each other [91]. This rises the question how a constant flow, transporting CSF away from the ventricular wall, would look in the natural, closed v3V. Liposomes injected into the near wall flow in the distributor region at the head of flow 6 and flow 7 were transported along the ciliary prescribed streamlines towards the posterior duct at both ZT10 and ZT23.5. These liposomes leave the v3V for the aqueduct (Fig. 3.2 G, H and Fig. 3.5 D). 

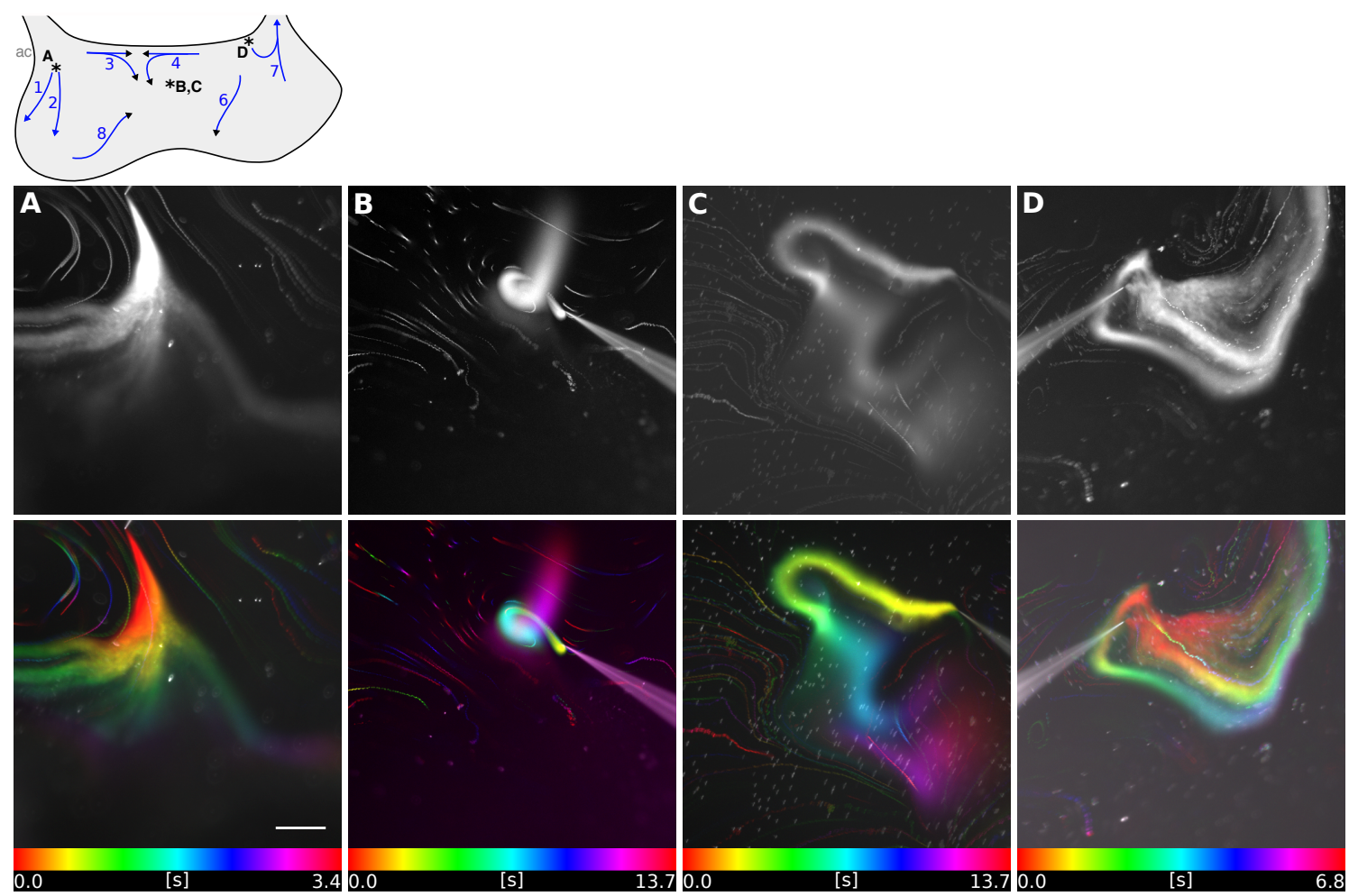

Figure 3.5: The propagation of fluorescent liposomes of composition I (Tab. 2.1) was studied by injecting small amounts into the ciliary near wall flow of the v3V. The propagation of liposomes was recoded over time and projected using Fiji [78]. The projections give an impression about the relative concentration of liposomes while transported along the ciliary flow. Additionally, a temporal color code was applied. The small schema of the $\mathrm{v} 3 \mathrm{~V}$ above $\mathbf{A}$ illustrates the position of local liposome application (*) shown in $\mathbf{A}$ to $\mathbf{D}$. A liposome propagation in flow 1, 2 and 3 at ZT 23.5. B liposome propagation underneath the separatrix at ZT 23.5. The liposomes circled around and then were transported out of the focal plane. $\mathbf{C}$ liposome propagation underneath the separatrix at ZT10. The liposomes were transported along flow 4 and then downwards in the direction of flow 5. D liposome propagation along flow 7 in the direction of the posterior duct at ZT 23.5. Scale bar $=100 \mu \mathrm{m}$, [s] = seconds

\subsection{Liposome interaction with ependymal cilia}

The preceding studies demonstrated, that liposomes in the ventral third ventricle are transported along precise trajectories, which are generate by beating cilia. Back and forth moving but stationary fluorescent spots were observed on the apical side of ependymal cells after the injection of fluorescently-labeled liposomes into the cilia-generated near wall flow (Movie M1, Fig. 3.6 A). At higher magnification, these moving fluorescent spots were identified as liposomes attached to beating cilia bundles (Movie M2, Fig. 3.6 
B). These results suggest an interaction between liposomes and certain v3V ependymal cilia. The injection studies further showed that liposomes attached to beating cilia bundles while being transported along cilia prescribed trajectories. This observation was further analysed to potentially identify a target for extracellular vesicle-like cargo.
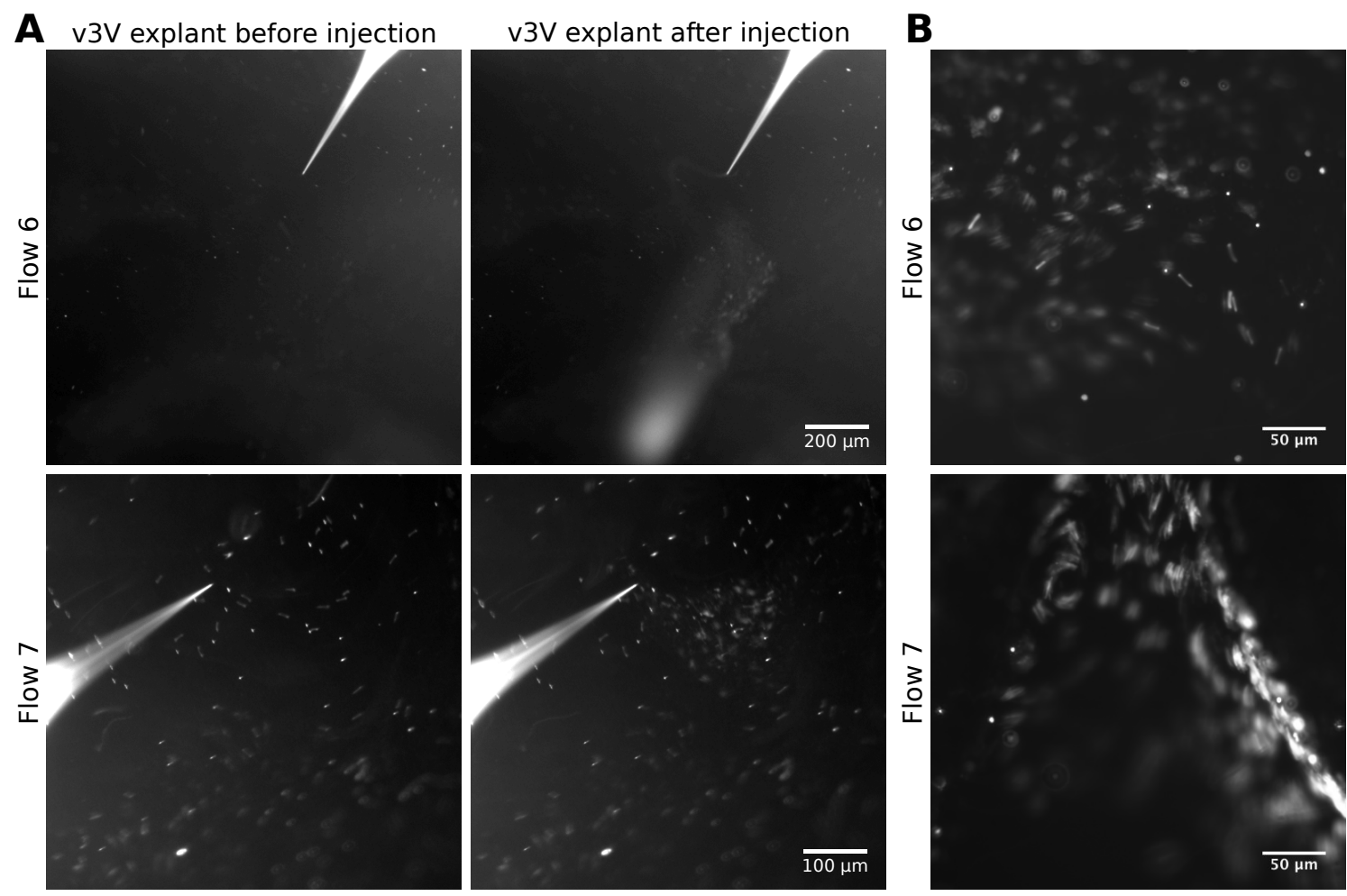

Figure 3.6: Liposome attach to structure on the apical side of v3V ependymal cells. A Liposomes of composition I (Tab. 2.1) are applied to the cilia-generated near wall flow in flow region 6 and 7 using a capillary. Following the transport of liposomes over certain areas fluorescent spots are visible beside fluorescent beads stuck on the apical ependymal cell surface. B At higher magnification fine frayed structures are visible on the apical side of the ependymal cells, which move back and forth (Movie M2). This indicates that, liposomes attach to beating cilia bundles.

\subsubsection{Liposome attachment to ependymal cells and cilia in the ventral third ventricle}

Potential sites that capture extracellular vesicle-like cargo were investigated by exposing v3V explants to homogeneous fluorescently-labeled liposome solution (composition II Tab. 2.1 and VIII Tab. 2.2). Prior to the v3V incubation in homogeneous liposome solution, the cilia-generated near wall flow was recorded by applying fluorescent beads. 
The corresponding flow maps were made from maximum intensity projections of recorded movies of bead movement $3.7 \mathbf{A}$ and $\mathbf{B}$. Using these flow maps a direct correlation of strong liposome attachment sites in the $\mathrm{v} 3 \mathrm{~V}$ with the corresponding cilia-generated flow regions can be made by comparing the position of stationary fluorescent beads stuck to the ependymal surface.

After incubating the tissue explant in a 1:500 diluted liposome solution for $10 \mathrm{~min}$ at room temperature (RT) a heterogeneous distribution of fluorescently-labeled liposomes attached to the ependymal cell cilia and surface was observed (Fig. 3.7 C and D and 3.8 A). Cells of the surrounding brain parenchyma and damaged ependymal cells, which lay along the cutting line during the $\mathrm{v} 3 \mathrm{~V}$ preparation, showed a strong liposome attachment as well (Fig. 3.7 C and D). As negative control, v3V explants were incubated in DMEM at RT. In the negative control the fluorescent signal was neither observed on the v3V ependyma nor in the surrounding brain parenchyma (Fig. 3.7 $\mathbf{E}$ and $\mathbf{F}$ ).

The regions of stronger liposome attachment were identified as the area of flow 6 and flow 7 and proceeded along the posterior duct (App. Fig. 5.5). The attachment areas were similar for both hemispheres. The position of the region of flow 6 within the $\mathrm{v} 3 \mathrm{~V}$ was located by Faubel et al. [2] between Bregma level -1.2 and -1.6. This correlates with the position of the ventromedial nucleus and partly with the arcuate nucleus [92], two hypothalamic nuclei that may respond to CSF signaling.

\section{Influence of liposome attachment on cilia beating direction and frequency}

Cilia are known to have sensory functions and are able to respond to chemical as well as mechanical signals in their environment [31, 93, 94]. The strong attachment of liposomes to ependymal cell cilia in specific regions of the $\mathrm{v} 3 \mathrm{~V}$ raises the question whether the attachment might lead to a cellular response. For example, a change of the ciliary beating might result in a slightly different flow pattern. To investigate a possible influence of liposome attachment to ependymal cell cilia in the $\mathrm{v} 3 \mathrm{~V}$ on the cilia beating frequency and direction, the cilia-generated flow was recorded on the same tissue explant before and after the incubation in a homogenious liposome solution.

The v3V explant was freshly prepared and the cilia-generated flow was recorded using fluorescent beads as described in Sec. 2.4.1 and 2.4.2. Then the tissue explant was placed in a 1:500 diluted fluorescently-labeled liposome solution and incubated for $10 \mathrm{~min}$ at RT (see Sec. 2.6.3). Afterwards the tissue explant was briefly washed with DMEM and 
left hemisphere
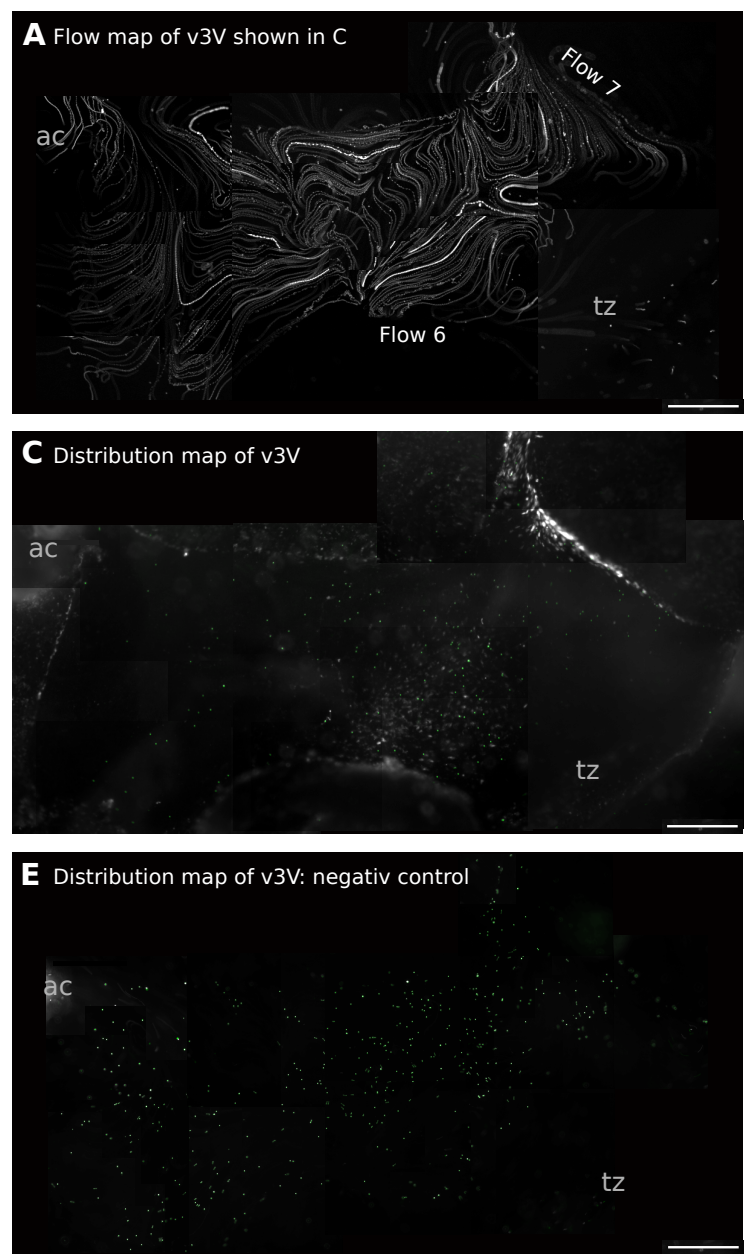

right hemisphere
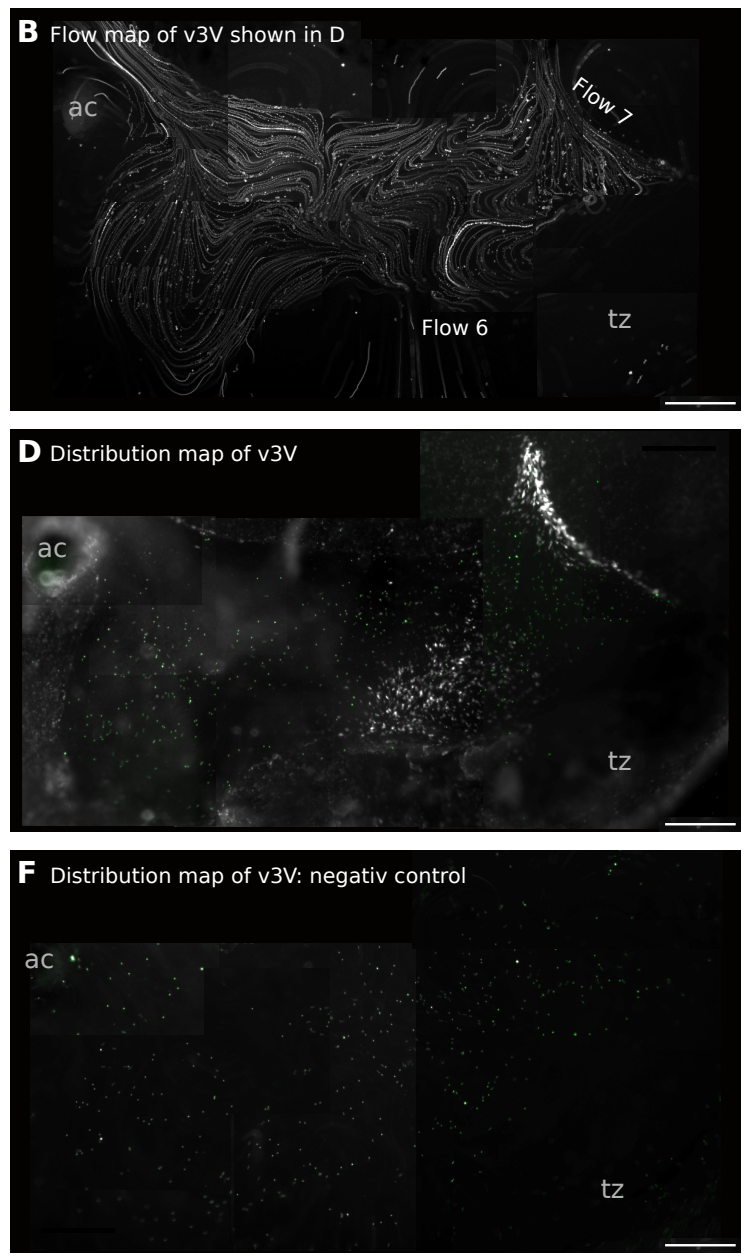

Figure 3.7: The cilia mediated bead flow of a freshly prepared v3V explant was recorded. Afterwards the $\mathrm{v} 3 \mathrm{~V}$ explant was incubated in fluorescence-labeled liposome solution of composition VIII in a dilution of 1 to 500 for $10 \mathrm{~min}$. A and $\mathbf{B}$ show a map of liposome attachment after incubation. Fluorescence-labeled liposome are shown in white, fluorescent beads are shown in green. $\mathbf{C}$ and $\mathbf{D}$ show the corresponding flow maps to $\mathbf{A}$ and $\mathbf{B}$. The left and right hemisphere show a stronger attachment in the region of flow 6 and flow 7 close to the posterior duct. As control v3V explant was incubated in DMEM for $10 \mathrm{~min}$. The incubation maps of the controls are shown in $\mathbf{E}$ and $\mathbf{F}$. Scale bar $=300 \mu \mathrm{m}, \mathrm{ac}=$ anterior commissure, $\mathrm{tz}=$ tanycyte region.

placed back into the petri dish prepared for recording the cilia-generated flow. Then, the cilia driven flow was recorded again at two time points after the incubation. First directly after the incubation and then, at least an hour after the incubation. As control a $\mathrm{v} 3 \mathrm{~V}$ explant was processed under the same conditions but incubated in DMEM without liposomes. 

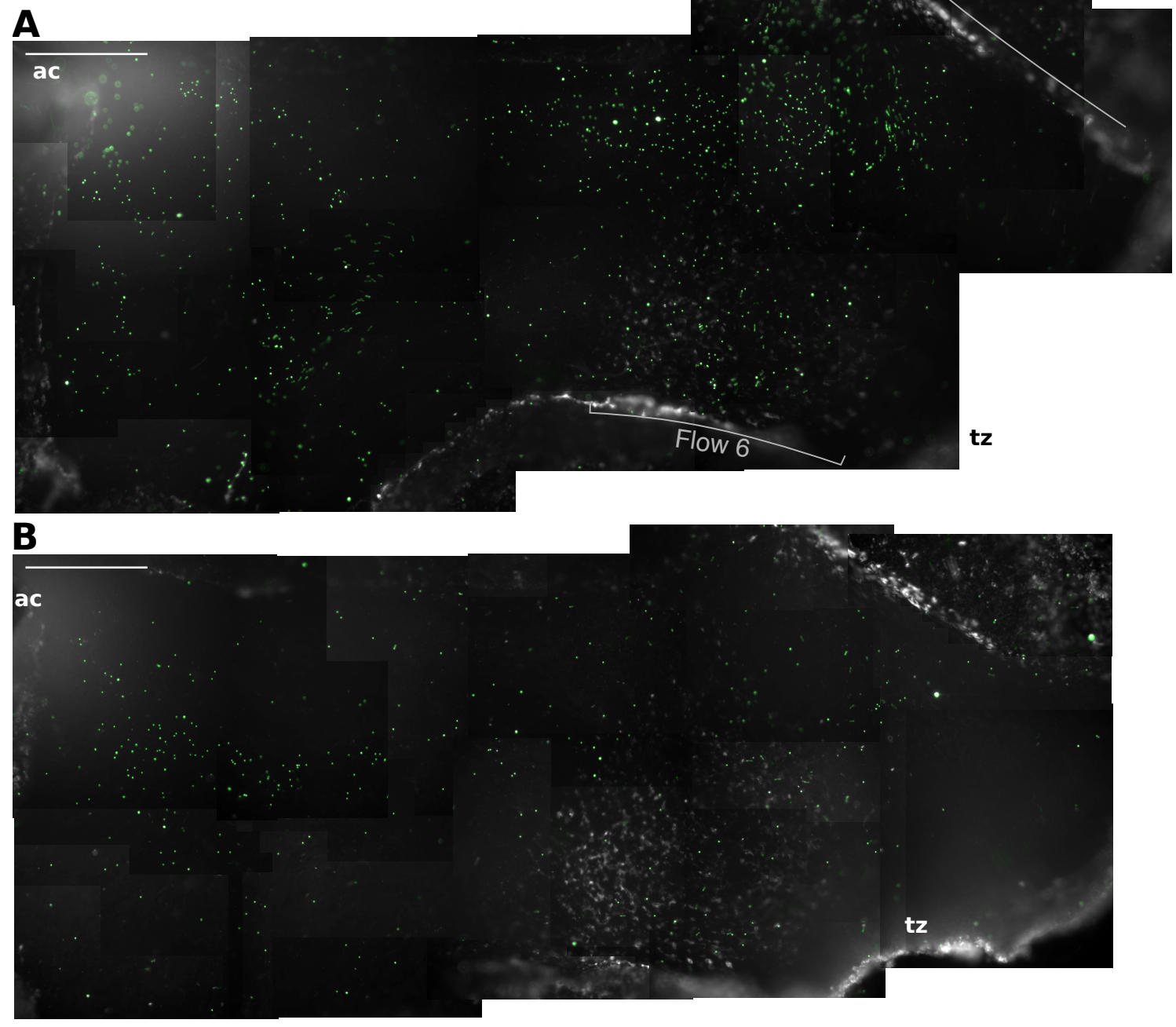

Figure 3.8: V3V explants were incubated in fluorescence-labeled liposome solution in a dilution of 1:500 for $10 \mathrm{~min} \mathrm{~A}$ and $30 \mathrm{~min} \mathbf{B}$. Liposomes were of composition II (Tab. 2.1) and prepared by size exclusion chromatography. The fluorescence signal of liposomes is shown in white, fluorescent beads are shown in green. Liposomes attached strongly in the region the of flow 6 and in flow 7 along the posterior duct. Light gray lines along the v3V border indicate the position of flow 6 and flow 7. Cells of the surrounding brain parenchyma show strong liposome attachment. Scale bar $=300 \mu \mathrm{m}, \mathrm{ac}=$ anterior commissure, $\mathrm{tz}=$ tanycyte region. 
The cilia-generated flow pattern of each sample and time point was analyzed by using maximum intensity projections (see 2.4.3). Figure 3.9 A shows the flow patterns of a $\mathrm{v} 3 \mathrm{~V}$ explant before and after the incubation in homogeneous liposome solution. The flow patterns of the control are shown in Fig. 3.9 B. Neither the cilia-generated flow of the sample incubated in liposome solution nor the control showed an overt change in the cilia-driven flow direction over the observed period of time. Thus, the cilia beating direction does not seem to be influenced by liposome attachment or the tissue handling during the incubation process.

During the visualization and recording of the cilia-driven near wall flow over the course of the incubation experiments a decrease of the bead transport velocity was observed. The transport velocity of beads depends on the flow velocity of the cilia-generated near wall flow and consequently on the cilia beating frequency. Thereby, a decrease in the bead transport velocity could indicate an effect of liposome attachment on the cilia beating frequency.

To investigate the effect, the bead transport velocities were calculated and used to determine the bead velocity distribution on each $\mathrm{v} 3 \mathrm{~V}$ tissue explant at the three different time points during the incubation experiment (cf. cilia-generated flow pattern comparison above). The bead velocities were calculated before, directly after and again at least an hour after the incubation process. The v3V tissue explant was either incubated in a diluted solution of fluorescently-labeled liposomes for $10 \mathrm{~min}$ at RT (Fig. 3.10 A to D left column) or in liposome free DMEM under the same conditions (Fig. 3.10 A to D right column). The velocity distribution was determined in flow region 1 and 2, flow region 4 , flow region 6 and flow region 7.

The velocity distributions of all flow regions show a decrease of the bead transport velocity directly after the incubation process (Fig. 3.10 A to $\mathbf{D}$ row one and two). This finding was independent of the presence of liposomes during the incubation process. Interestingly, the bead velocity decrease was less prominent in the region of flow 7 , while all other regions were affected more strongly. The mean velocity $\bar{v}$ was reduced by $81 \%$ (left column) and $78 \%$ (right column) in flow region 1 and 2, by $60 \%$ and $47 \%$ in flow region 4 , by $72 \%$ and $64 \%$ in flow region 6 and $21 \%$ and $14 \%$ in flow region 7 . An hour to one and a half after the incubation process the bead transport velocity increased again in all flow regions. This was independent of the presence of liposomes during the incubation process. The mean velocity of each flow region did not reach the initial value before the incubation process, except for flow region 7 , which increased even further. These results 
suggest, that the observed changes of bead transport velocity during an incubation experiment are not caused by liposome attachment during the incubation process. Rather, these changes seem to be a consequence of the tissue handling during the incubation process.

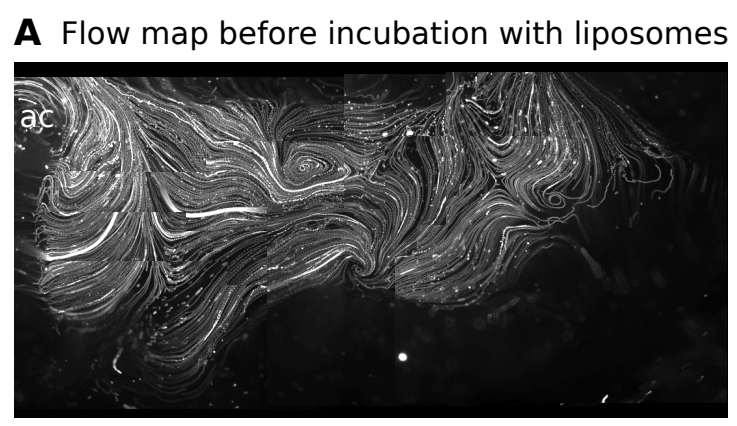

Flow map after incubation

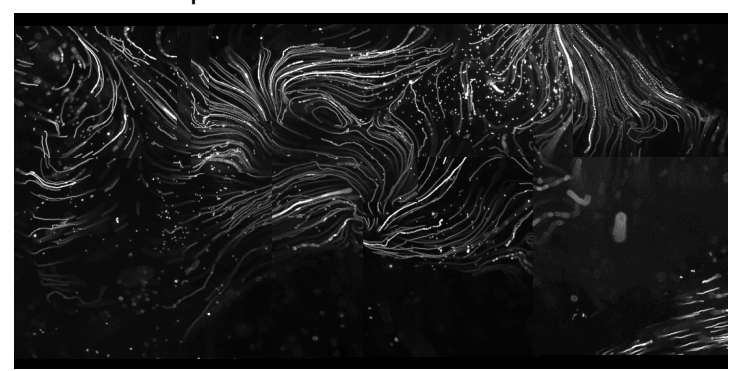

Flow map 1.5h after incubation

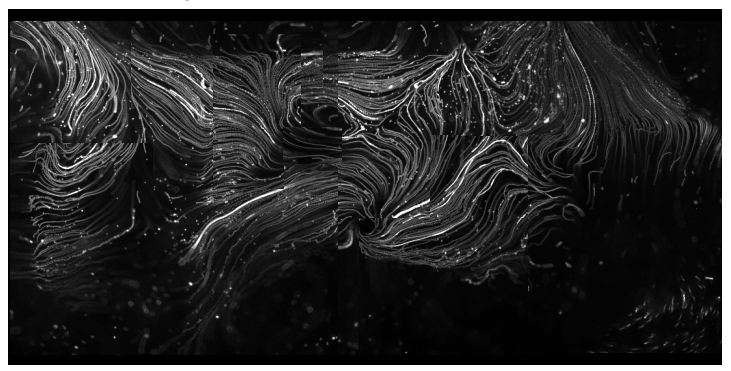

B Flow map before incubation in DMEM

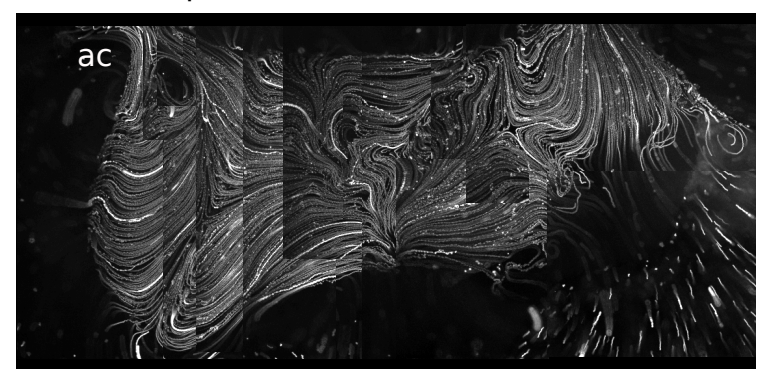

Flow map after incubation in DMEM

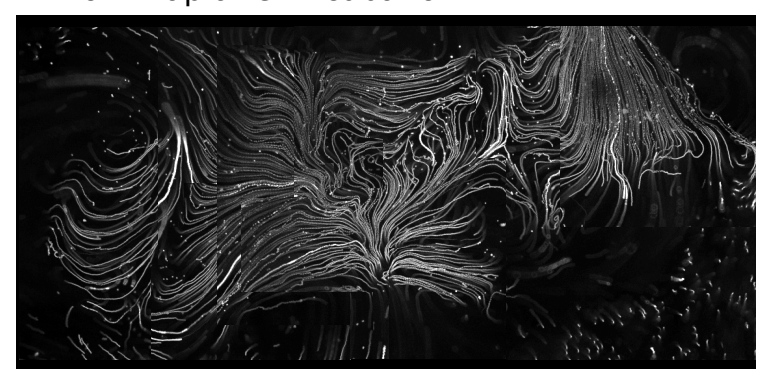

Flow map $1 \mathrm{~h}$ after incubation in DMEM

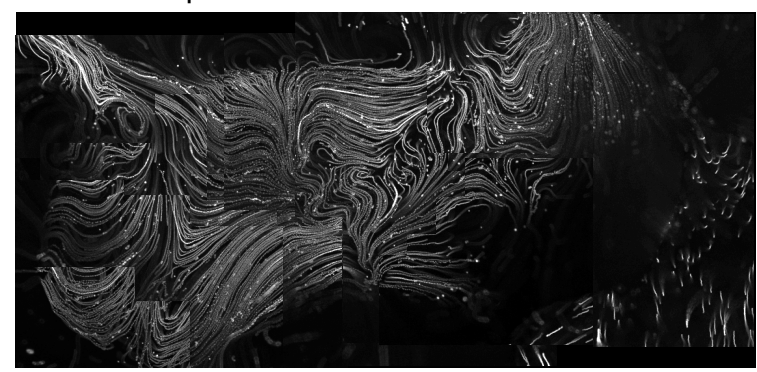

Figure 3.9: Comparison of cilia-generated flow pattern on the same v3V tissue explant before and after incubation experiment. The cilia driven flow was visualized using fluorescent beads and recorded as described in section 2.4.2. The flow pattern was analyzed as described in section 2.4.3. The tissue explant was either incubated in a homogeneous solution of diluted fluorescently-labeled liposomes (A) or without liposomes in DMEM (B). A The cilia-generated flow was recorded and thereafter the v3V tissue explant was incubated in a diluted solution of fluorescently-labeled liposomes for $10 \mathrm{~min}$ at RT. Then the cilia driven flow was recorded directly after the incubation and again one and a half hours after the incubation process. B The cilia-generated flow of the control was recorded and the tissue explant was incubated in DMEM for $10 \mathrm{~min}$ at RT. The cilia-generated flow was recorded directly after and then one hour after the incubation. Neither after the incubation in diluted liposome solution nor in DMEM the flow patterns and flow directions show overt changes over time. 
To test whether liposome attachment influences the cilia beating frequency in the v3V, the ciliary beating was recorded from a lateral view by DIC microscopy before and after adding fluorescently-labeled liposomes (Sec. 2.7.2). The tissue was not transferred and always kept in a sufficient amount of medium. The ciliary beating was recorded with $200 \mathrm{fps}$ and the beating frequency of single bundles calculated by using the Fiji Kymograph plugin [78]. This experiment was performed in collaboration with the lab of Dr. Cecilia Lo of the University of Pittsburgh. The resulting frequency distributions are shown in Fig. 3.11. The cilia beating frequency of tissue 1 before adding liposomes varied from $6 \mathrm{~Hz}$ to $24 \mathrm{~Hz}$ with an average value of $(11.7 \pm 3.7) \mathrm{Hz}$. Afterwards the beating frequency varied from $8 \mathrm{~Hz}$ to $18 \mathrm{~Hz}$ with an average value of $(13.1 \pm 3.0) \mathrm{Hz}$. Tissue 2 showed cilia beating frequencies from $12 \mathrm{~Hz}$ to $26 \mathrm{~Hz}$ with an average of $(18.5 \pm 3.4) \mathrm{Hz}$ before and $18 \mathrm{~Hz}$ to $24 \mathrm{~Hz}$ with an average of $(21.4 \pm 1.4) \mathrm{Hz}$ after adding liposomes. Although the sample size is too small to exclude an influence of liposome attachment on the beating of cilia completely, this data suggests that the cilia beating frequency is not affected by the presence and attachment of liposomes. In addition to the results of the bead transport velocity analysis, this result suggests, that the ependymal cilia beating frequency is not influenced by liposome attachment. 
A Incubation Exp.: + Lipo Incubation Exp: - Lipo

B Incubation Exp.: + Lipo Incubation Exp: - Lipo

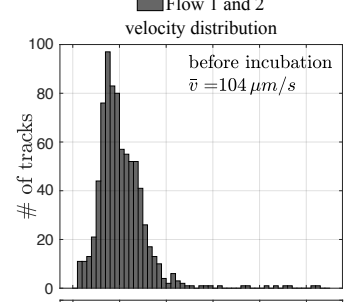

velocity distribution
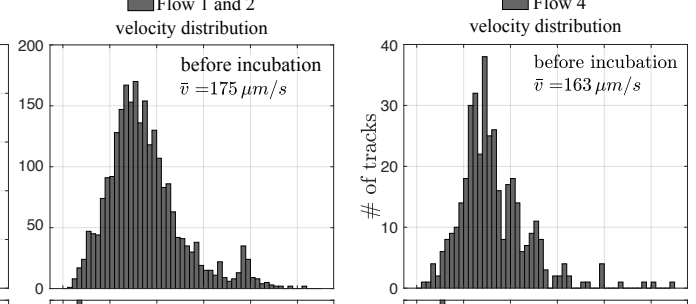

Flow 4
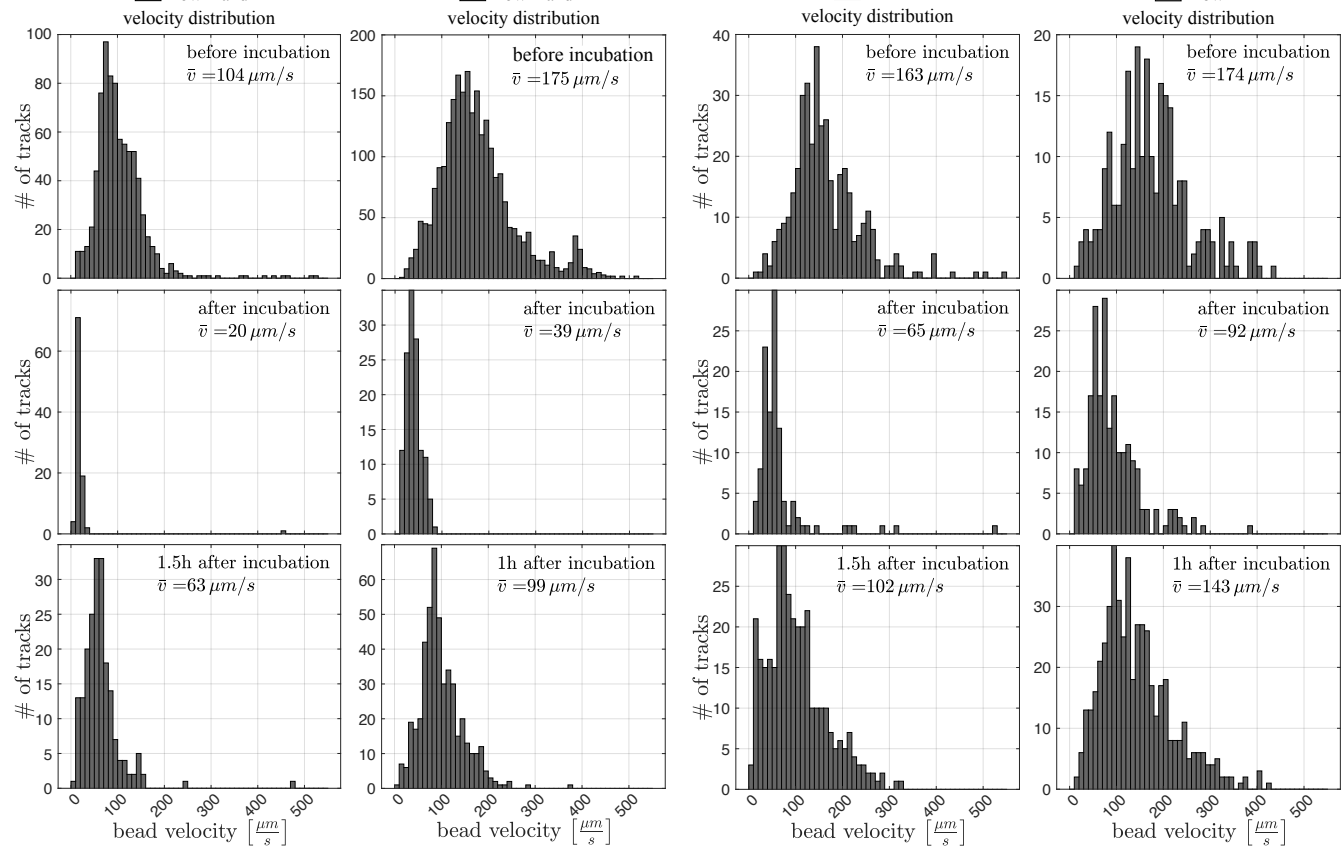

C Incubation Exp.: + Lipo Incubation Exp.: - Lipo

D Incubation Exp.: + Lipo

Incubation Exp.: - Lipo
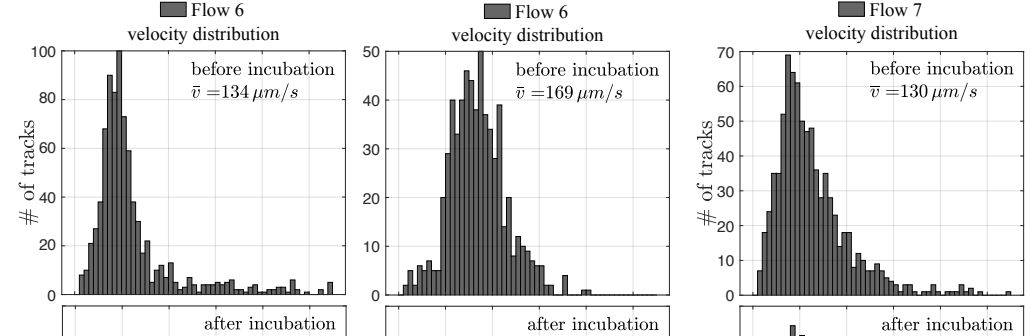

Flow 7
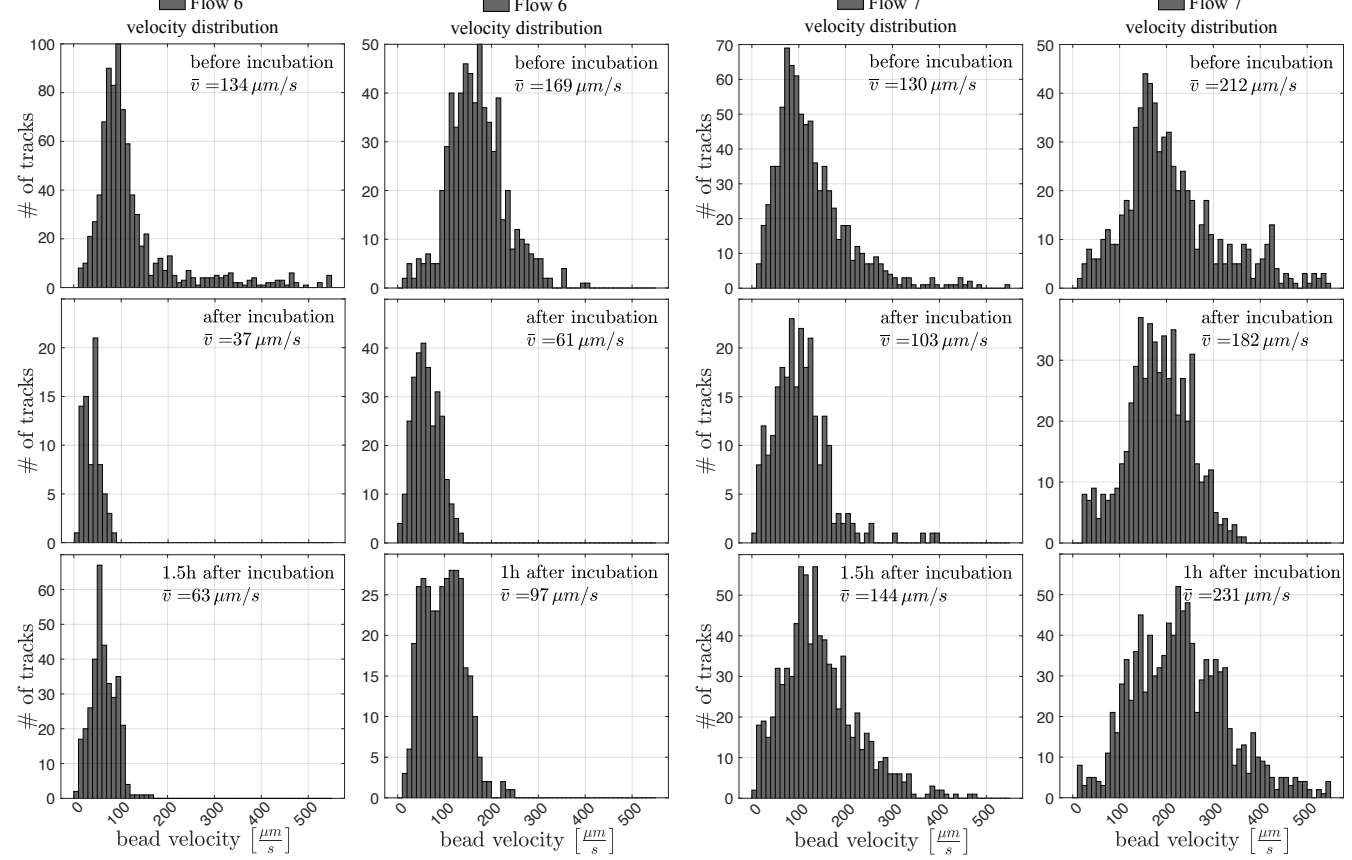

Figure 3.10: The velocity distribution of fluorescent beads transported in the cilia-generated flow along the $\mathrm{v} 3 \mathrm{~V}$ walls over the course of an incubation experiment (see Sec. 2.6.3). The bead velocities were calculated at three time points during the incubation experiment on the same tissue explant. First, before the incubation process, second directly after the incubation process and third at least an hour after the incubation process. The velocities were calculated in flow region 1 and $2(\mathbf{A})$, region $4(\mathbf{B})$, region $6(\mathbf{C})$ and region 7 (D) using particle tracking. The tissue explants were either incubated in a diluted solution of fluorescently-labeled liposomes (+ Lipo) or DMEM without liposomes (- Lipo) during the incubation process. $\bar{v}$ shows the mean $54^{\text {velocity. }}$ 
Cilia beating frequencies before and after adding liposomes
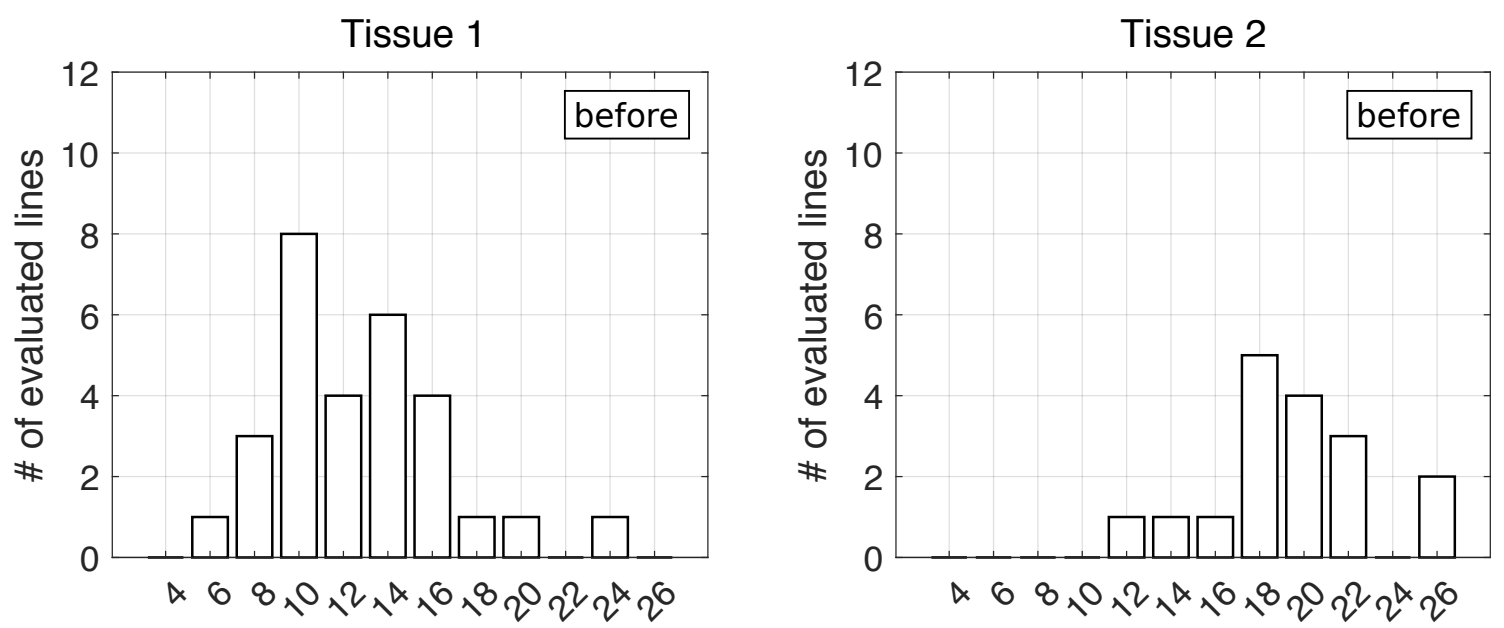

Cilia beating frequency $[\mathrm{Hz}]$

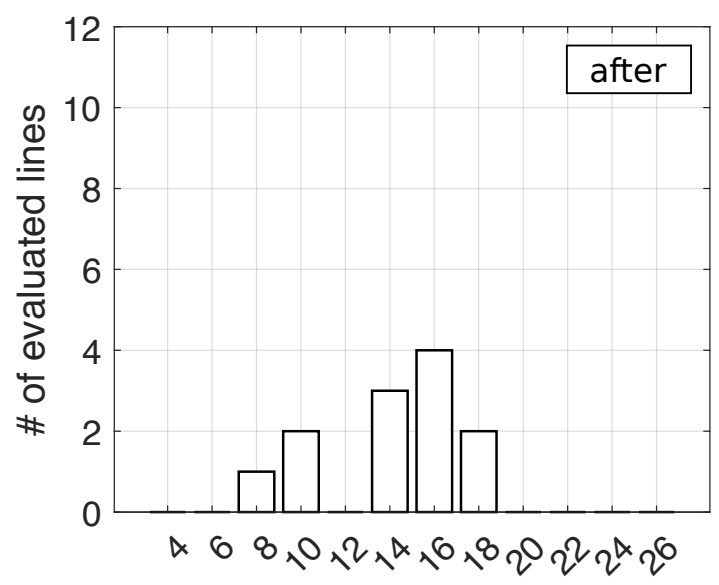

Cilia beating frequency $[\mathrm{Hz}]$

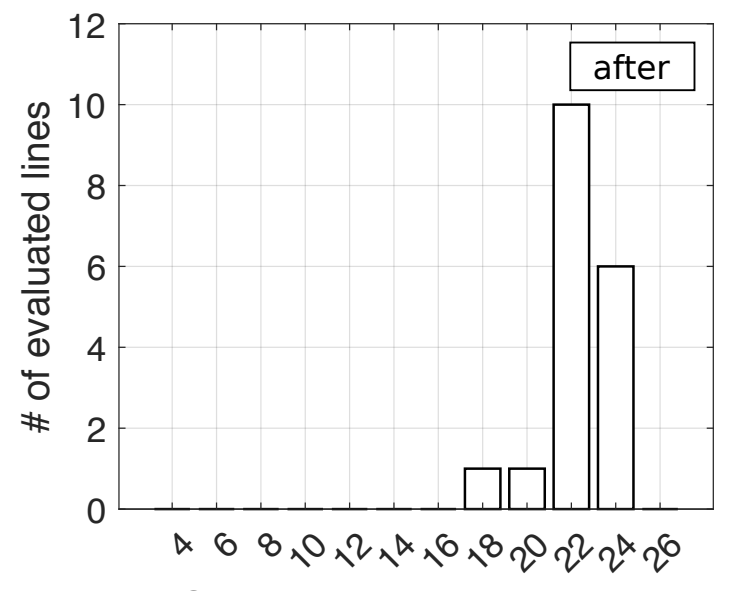

Cilia beating frequency $[\mathrm{Hz}]$

Figure 3.11: Cilia beating frequency distribution in the v3V before and after adding fluorescentlylabeled liposomes. The cilia beating in the $\mathrm{v} 3 \mathrm{~V}$ was recorded from a lateral perspective with $200 \mathrm{fps}$ before and after adding liposomes for each tissue. The cilia beating frequency was measured by making kymographs for several cilia bundles per recorded movie using Fiji [78]. The top row shows the cilia beating frequency of tissue 1 and tissue 2 before the addition of liposomes. The bottom row shows the cilia beating frequency of tissue 1 and tissue 2 after adding liposomes. 


\section{Position of liposome attachment along the cilia bundle}

For a precise localization of the liposome attachment site within a beating cilia bundle confocal microscopy was used (Sec. 2.6.4). A v3V explant was incubated in diluted, fluorescently-labeled liposome solution (dilution 1:400) at RT. Then the tissue explant was transferred to a prior prepared, DMEM filled imaging chamber with the ependymal wall facing downwards. The chamber was placed in an inverted confocal microscope and the movement of beating cilia was imaged with $117 \mathrm{fps}$. Starting at the ependymal cell surface and moving towards the cilia tip, the fluorescent signal from fluorescently-labeled liposomes was recorded over time. Such stacks were recorded in flows 6 and 7, which show strong liposome attachment. Single images from representative confocal stacks are shown in Fig. 3.12 A and B. Liposomes attached to cilia bundles were periodically moving along particular trajectories. With growing distance from the ependymal surface the number of fluorescent spots and their fluorescent intensity increased. This experiment was performed in collaboration with the lab of Dr. Cecilia Lo of the University of Pittsburgh.

The periodic movement of attached liposomes was used to calculate the cilia beating frequency by plotting the changing intensity along the ciliary beating trajectory as kymographs (Fig. 3.12 C). These kymographs reflect the increasing fluorescent intensity towards the cilia tips. These results suggest, that liposomes predominantly attach to the ciliary tip.

The cilia beating frequencies calculated from the recorded image stacks are plotted in Fig. 3.13. The calculated average beating frequency in flow 6 was $(3.23 \pm 1.7) \mathrm{Hz}$ and $(17.7 \pm 5.8) \mathrm{Hz}$ in flow 7 . The beating frequencies measured in flow 6 are much lower than the frequencies measured in flow 7. This may be caused by transferring the tissue directly before the imaging, which effects flow 6 stronger than flow 7 as shown in the section above. The frequencies measured for flow 7 are consistent with previous results $[90,95]$. 
A Flow 6: liposomes attached to beating cilia
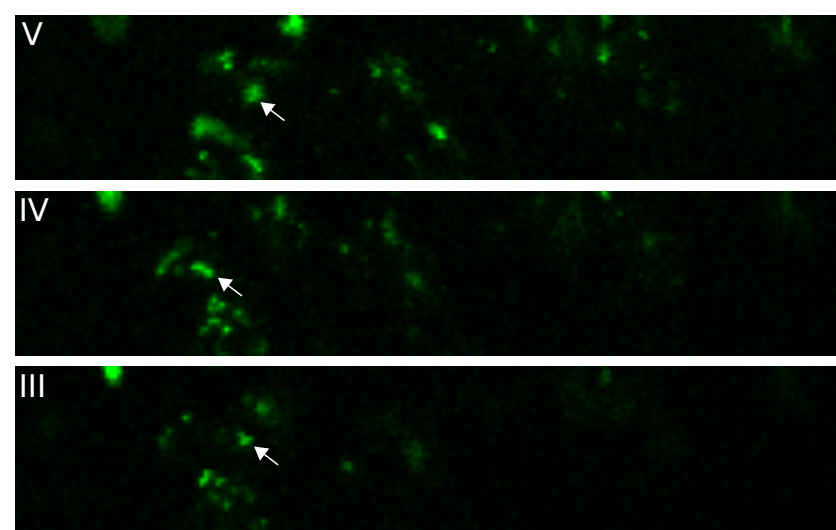

II
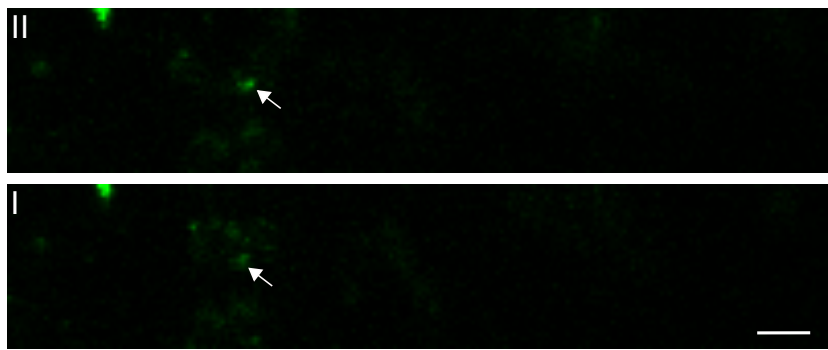

B Flow 7: liposomes attached to beating cilia
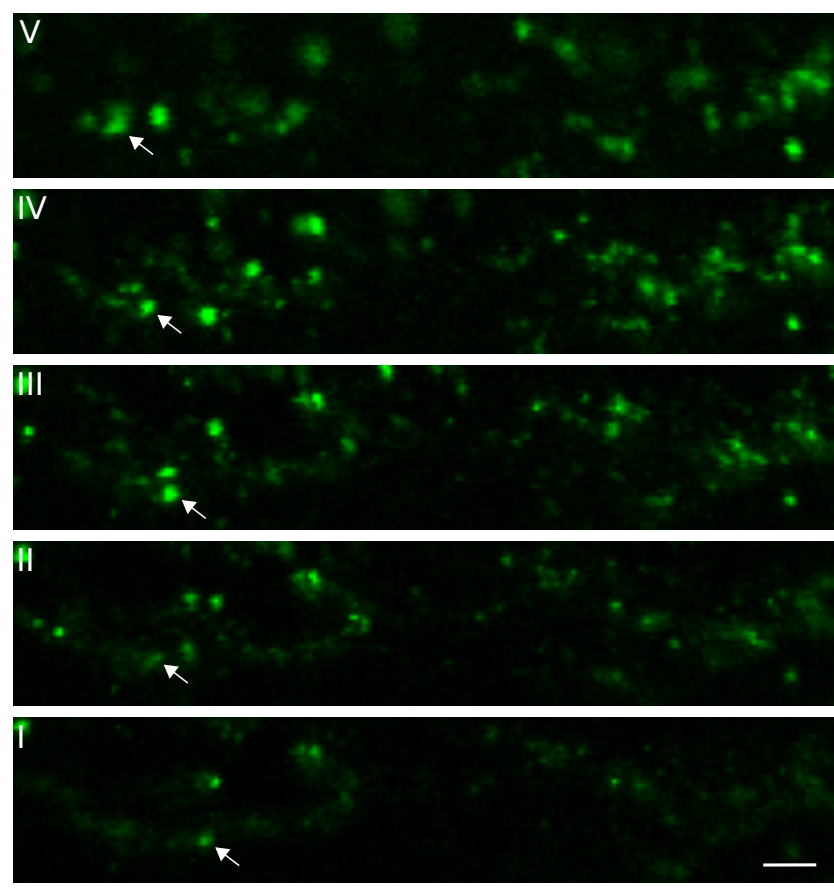

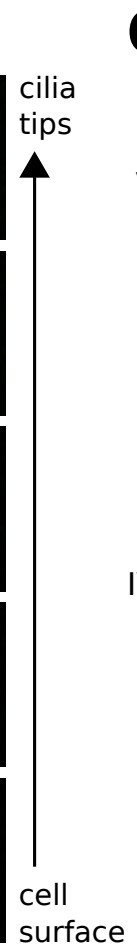

cilia tips

surface

\section{C flow 6}

Flow 7

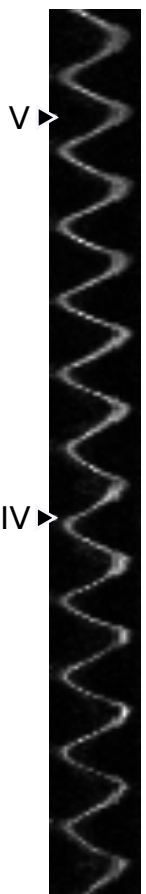

III)

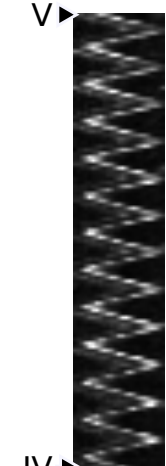

$\rightarrow$

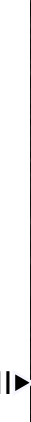

III

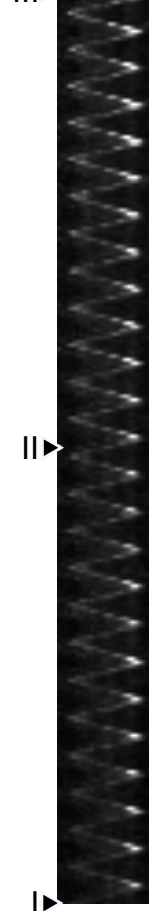

Figure 3.12: Analysis of liposome attachment position on beating cilia relative to ependymal cell surface. A and $\mathbf{B}$ show representative images of a confocal $\mathrm{z}$ stack imaged over time. The imaging started at the ependymal cell surface and moved towards of the cilia tips. White arrows indicate the position of liposomes attached to one particular cilia bundle. The kymographs of these bundles are shown in $\mathbf{C}$ and cover a time span of $4.27 \mathrm{~s}$. Roman numerals refer to images shown in $\mathbf{A}$ and $\mathbf{B}$. Scale bar $=10 \mu \mathrm{m}$. 

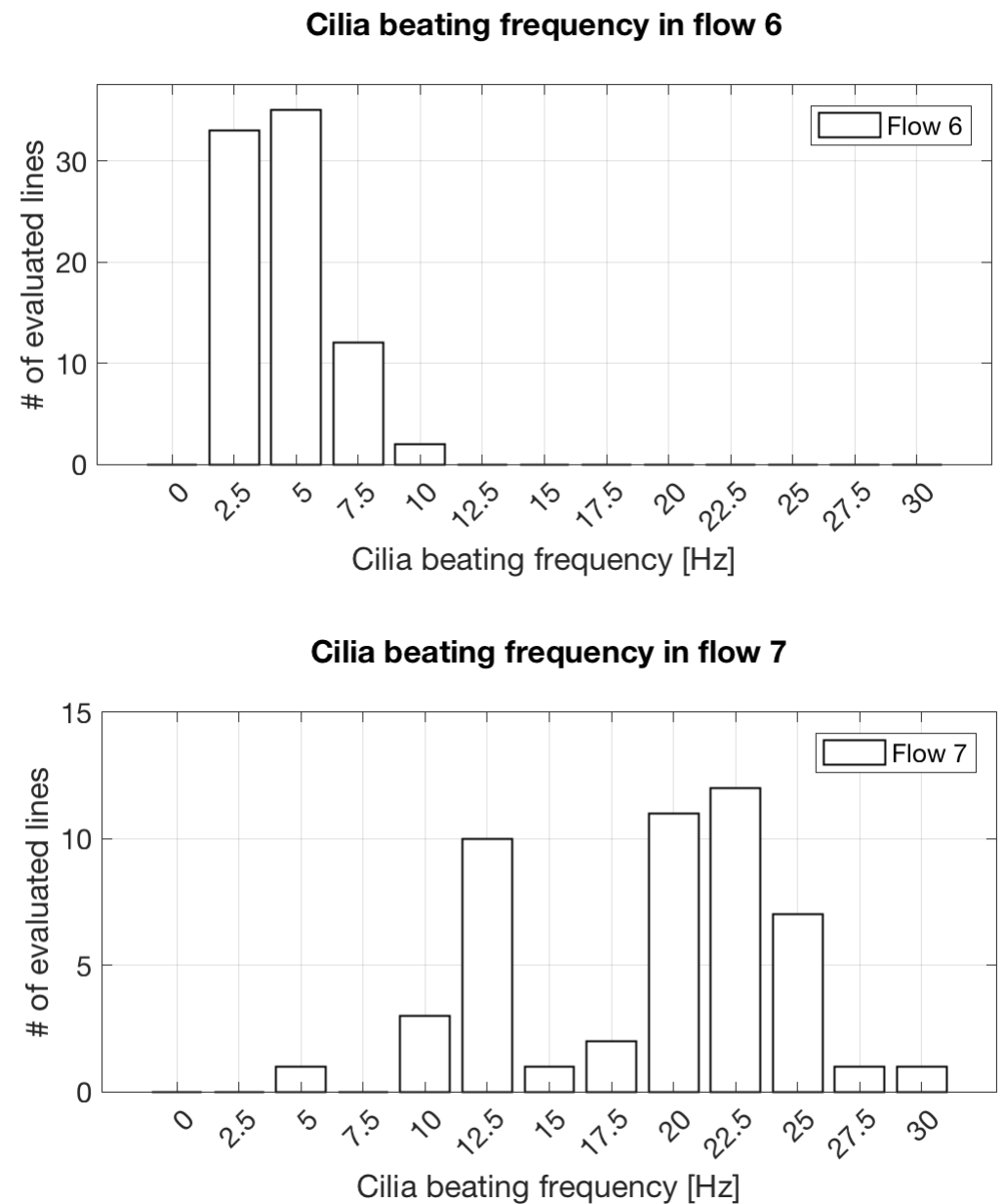

Figure 3.13: Liposome attachment to cilia bundles was used to visualize the ciliary beating in flow 6 and flow 7 and to calculate the cilia beating frequency. V3V sample was incubated in a diluted solution of fluorescently-labeled liposomes. Confocal microscopy was used to record the movement of fluorescent liposomes attached to cilia over time. The cilia beating frequency was calculated from kymographs made for each periodic moving fluorescent signal. The resulting frequencies were sorted and plotted in a histogram separately for flow 6 and flow 7. The frequency values range from $2.5 \mathrm{~Hz}$ to $30.0 \mathrm{~Hz}$. 


\section{Attachment of liposomes to damaged cells}

Cells of the brain parenchyma outside the $\mathrm{v} 3 \mathrm{~V}$ area show a strong attachment of fluorescently-labeled liposomes over the whole cell membrane after incubation in a homogeneous solution of such (Fig. 3.7 C and D, App. Fig. 5.3 to 5.5). This also includes ependymal cells along the anterior to posterior cutting line. Ependymal cells untouched by the cutting tools used for the preparation of the $\mathrm{v} 3 \mathrm{~V}$ explant did not show liposome attachment over the whole cell membrane. This lead to the hypothesis that damaged cells may capture liposomes.

To test this hypothesis, a wound closure assay was performed using NIH-3T3 mouse fibroblasts and Z310 rat choroid plexus cells. Cells were grown on a cover slip until a confluent cell mono-layer had formed. Then the cell mono-layer was wounded by scratching the cell layer with a pipette tip. Afterwards the cells were incubated in a homogeneous solution of fluorescently-labeled liposomes, fixed and stained. Wounded NIH-3T3 cells showed a strong attachment of liposomes along the scratch line (App. Fig. 5.2), while the attachment to Z310 cells was rare and seemed unspecific over the whole cell layer. These results indicate, that wounded cells have a higher affinity to attach liposomes, but this process also seems to depend on the cell type.

The wound closure assay was performed by Dr. Zuzana Ditte from the Max-PlanckInstitut for Biophysical Chemestry.

\subsubsection{Liposomes-cilia interaction in dependence of liposome lipid-composition}

It was shown, that liposomes can attach to ependymal cell cilia while being transported along the cilia-driven near wall flow of the v3V. The overt transport of liposomes follows the cilia-generated flow streamlines, which seem to be uninfluenced by liposome attachment to the ependymal cilia. The attachment of liposomes is strongest in the region of flow 6, flow 7 and the posterior duct (Fig. 3.7 and App. Fig. 5.5). This was observed during the local application of fluorescently-labeled liposomes to the cilia-generated near wall flow, as well as after the exposure of v3V tissue explants to homogeneous liposome solution. Further the application of fluorescently-labeled liposomes to the near wall flow showed that the attachment process occurs within seconds (Fig. 3.6).

These results suggest an interaction between liposomes and ependymal cilia. However, the mechanism behind the attachment process is unclear. The interaction could be caused by interactions between the liposomal membrane and components of the cilia membrane. 
Indeed, the composition of the ciliary membrane is distinct from the plasma membrane they emerge from and varies with respect to the cilia function [40, 96, 97]. This also includes specific membrane proteins and lipids [40, 97], which may influence the surface charge. An interaction between a cilia specific component and the liposomes might explain the strong attachment affinity to ependymal cilia.

Previous studies $[10,73,98]$ also showed that the interaction of liposomes and cells depends on the liposomes lipid composition, which determines liposome properties like the rigidity and fluidity of the bilayer membrane and the surface charge. The latter can be affected by the presencs of charged lipids, like the negatively charged 1,2-dioleoyl-snglycero-3-phospho-L-serine (DOPS). To investigate the interaction between liposomes and ependymal cilia, fluorescently-labeled liposomes of various lipid compositions were prepared using either size exclusion chromatography or extrusion. Then, tissue explants of the v3V were incubated in homogeneous, diluted liposome solutions at room temperature. Afterwards each tissue explant was imaged using fluorescence microscopy (Sec. 2.6.3).

\section{Liposomes prepared with size exclusion chromatography}

Liposomes made from a lipid mixture, which contained DOPC, DOPE, DOPS and cholesterol (composition I and II Tab. 2.1), showed a strong attachment to ependymal cilia in region of flow 6 and flow 7 of the v3V. Individual components of this lipid composition are known to influence membrane properties or even cell signaling. For example DOPS is a type of phosphatidylserine. Phosphatidylserine is an anionic phospholipid that is involved in regulatory and signaling processes $[65,73]$. Further it was shown, that phosphatidylserine incorporated in the liposomal membrane is involved in the binding and uptake of liposomes by cells [98]. Also, cholesterol affects the mechanical properties of a membrane and is involved in signaling processes [99]. In addition to these components, the fluorescently-labeled DOPE lipid (DOPE-ATTO), that was used for the visualization of liposomes, was labeled with either an ATTO550 or an ATTO647N dye, which are both cationic $[100,101]$.

To investigate which component of the liposome lipid mixture causes the attachment to ependymal cilia, fluorescently-labeled liposomes were prepared with different lipid compositions, excluding components step by step (Tab. 2.1). To determine, whether the used head group labeled fluorescent DOPE-ATTO lipid contributes to the attachment, 
liposomes were prepared with fatty acid labeled NBD-PC as well. All liposomes were prepared with size exclusion chromatography ( Sec. 2.3.2). This preparation method was shown to lead to liposomes that vary from $20 \mathrm{~nm}$ to $45 \mathrm{~nm}$ in diameter, with the majority having a diameter around $30 \mathrm{~nm}$ (Fig. $3.1 \mathrm{E}$ ).

Tissue explants of the v3V were incubated in diluted homogeneous liposome solutions and the whole area of the $\mathrm{v} 3 \mathrm{~V}$ was searched for liposome attachment. Short movies were recorded in the region of flow 6 and flow 7. Afterwards these movies were projected using Fiji [78]. Representative images for each tested lipid composition are shown in Figure 3.14. Liposomes of each lipid composition attached strongly to ependymal cilia in flow region 6 and flow region 7 (App. Tab. 5.2). Additionally, slight liposome attachment to the ependymal cell surface and cells of the parenchyma was observed (Fig. 3.14, Fig. 3.8 and App. Fig. 5.3 and 5.4). Liposomes prepared with the fluorescent dye NBD were less bright and showed a weaker fluorescent signal. Moreover, NBD labeled liposomes showed fast bleaching.

Even though liposomes with different lipid compositions were tested regarding their interaction with ependymal cells in the $\mathrm{v} 3 \mathrm{~V}$, all of them showed attachment to ependymal cilia. However the preparation of liposomes using size exclusion chromatography also included the usage of the detergent sodium cholate [74] (Sec. 2.3.2). The detergent is needed for the preparation of liposomes with small diameters but could also influence the interaction between liposomes and ependymal cilia.

\section{Liposomes prepared with extrusion}

To determine wherever the presence of the detergent sodium cholate influences the interaction between liposomes and ependymal cilia, liposomes were prepared using the extrusion method without any detergent (Sec. 2.3.2). Liposomes were prepared with two different lipid compositions: Composition VIII, which contained DOPC, DOPE, DOPS, cholesterol and head group labeled DOPE-ATTO, and composition IX, which contained DOPC and DOPE-ATTO (Tab. 2.2). The size distributions were measured using NTA and resulted in a sharp peak around $80 \mathrm{~nm}$ (Fig. 3.1 A and B).

Tissue explants of the v3V were incubated in diluted, homogeneous liposome solution at RT. After the incubation process the whole area of the $\mathrm{v} 3 \mathrm{~V}$ was searched for liposome attachment and short movies were recorded in the region of flow 6 and flow 7. Each recorded movie was projected using Fiji [78]. Representative images are shown in Figure 
3.15. Strong liposome attachment to ependymal cilia was observed in the region of flow 6 and flow 7 for liposomes of lipid composition VIII. Additionally, slight liposome attachment was observed to the ependymal cell surface and cells of the surrounding parenchyma (Fig. 3.15 and App. Fig. 5.5). Liposomes of composition IX did not show strong attachment to ependymal cilia over the whole area of the v3V (Fig. 3.15 and App. Fig. 5.6). Although, liposome attachment to cells of the surrounding parenchyma was observed.

\section{Comparing liposome-cilia interaction depending on liposome lipid composition}

To investigate the influence of individual components in the liposome lipid mixture on the liposomes ependymal cilia interaction, liposome attachment in the $\mathrm{v} 3 \mathrm{~V}$ was compared between several lipid-compositions. Tissue explants of the v3V were incubated in homogeneous solutions of fluorescently-labeled liposomes, which were prepared with different lipid-compositions using size exclusion chromatography or extrusion.

Although the brightness of the fluorescent signal differed between liposomes visualized with head-group labeled DOPE-ATTO and fatty-acid labeled NBD-PC (composition II, V and VI), no overt difference regarding the liposome-cilia interaction in the $\mathrm{v} 3 \mathrm{~V}$ was observed (Fig. 3.14). Additionally, liposomes of composition IX, which were visualized with DOPE-ATTO and prepared with extrusion, did not show strong attachment to ependymal cilia (Fig. 3.15). Therefore, a dye induced interaction between liposomes and ependymal cilia seems unlikely.

All liposomes prepared with size exclusion chromatography showed attachment to ependymal cilia in the region of flow 6 and flow 7 of the v3V and to cells in the surrounding parenchyma (Fig. 3.14). This result suggests, that liposomes whose lipid mixture contains either DOPS or cholesterol in addition to cholate, due to the method of preparation, interact with ependymal cilia of the $\mathrm{v} 3 \mathrm{~V}$. Liposomes that were prepared using the extrusion method did not contain cholate. Strong liposome attachment to ependymal cilia was observed for liposomes of composition VIII (Fig. 3.15), which contains DOPS and cholesterol. In contrast, a strong attachment to ependymal cilia was not observed for liposomes of composition IX, which contain neither DOPS nor cholesterol. These results show, that either DOPS or cholesterol or both lead to liposome attachment in the v3V. Furthermore, cholate cloud influences the interaction as well. These results indicate, that the cilia membrane composition of ependymal cells differs in some regions of the $\mathrm{v} 3 \mathrm{~V}$. 
3.2 Liposome interaction with ependymal cilia
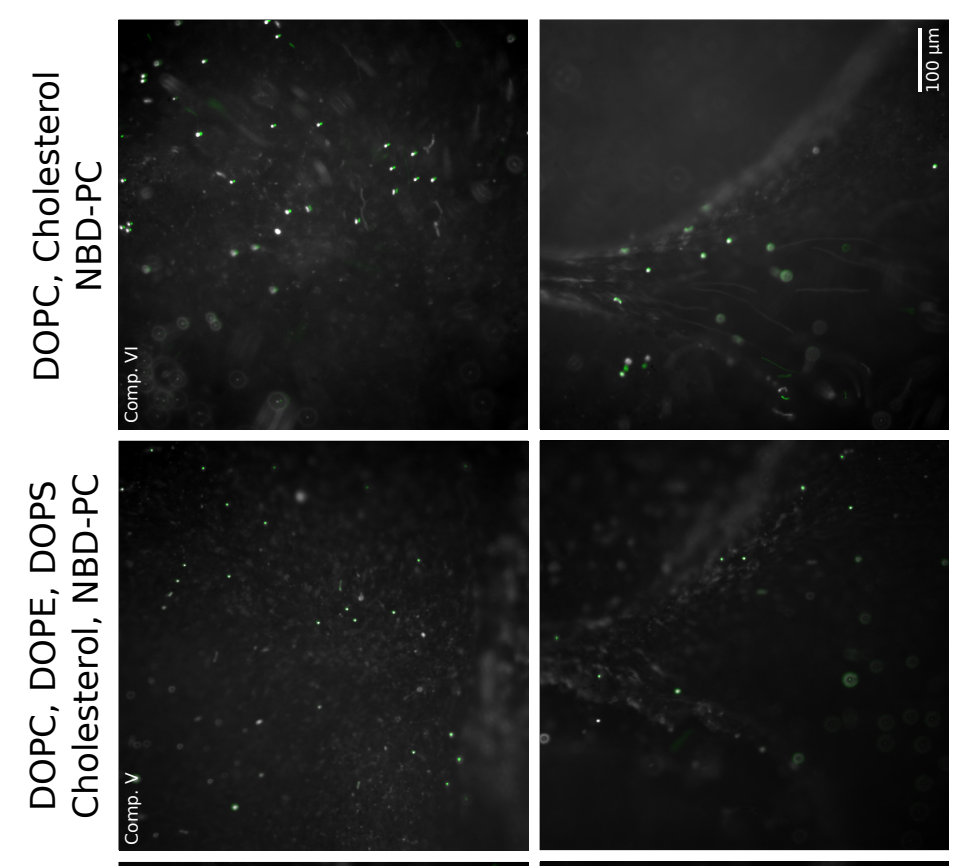

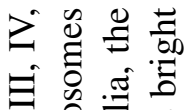

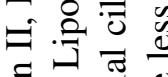

.

可

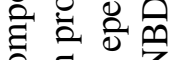

○ ฮี

귤
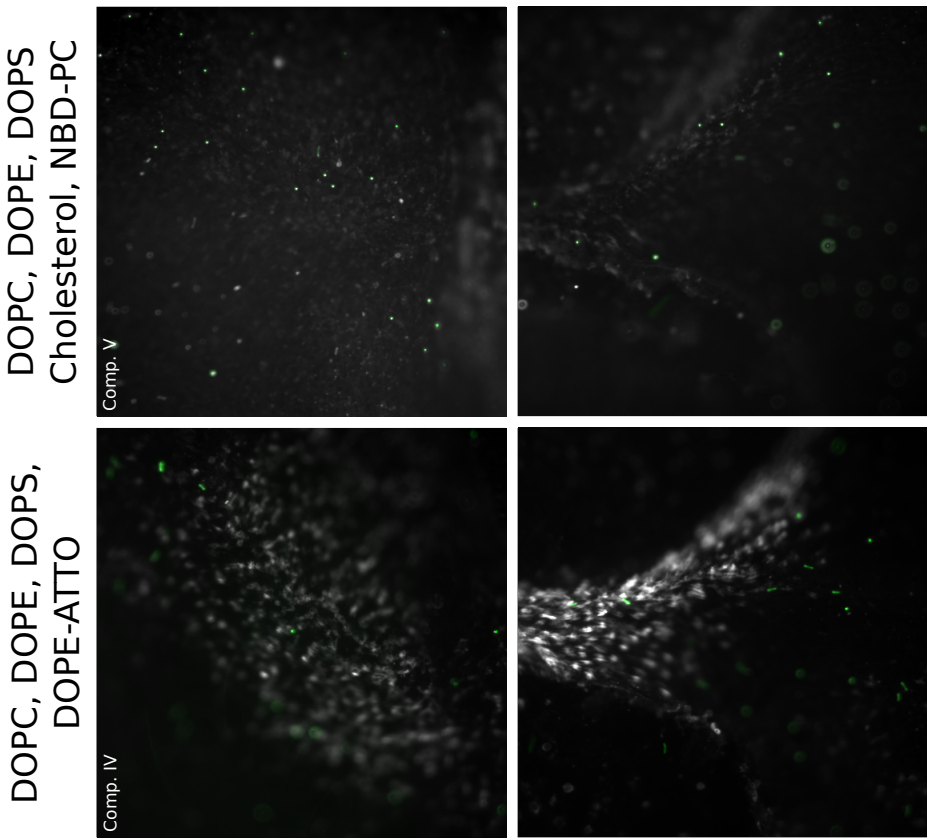

Ч.

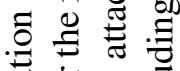

竞苛

or 5

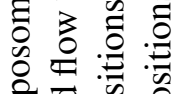

$\because$ :

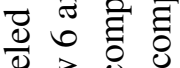

总 合

눙

ปี के

क. ह छ ฮี

응 0

怘怘芯

몽ㅇㅇ

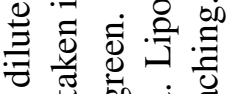
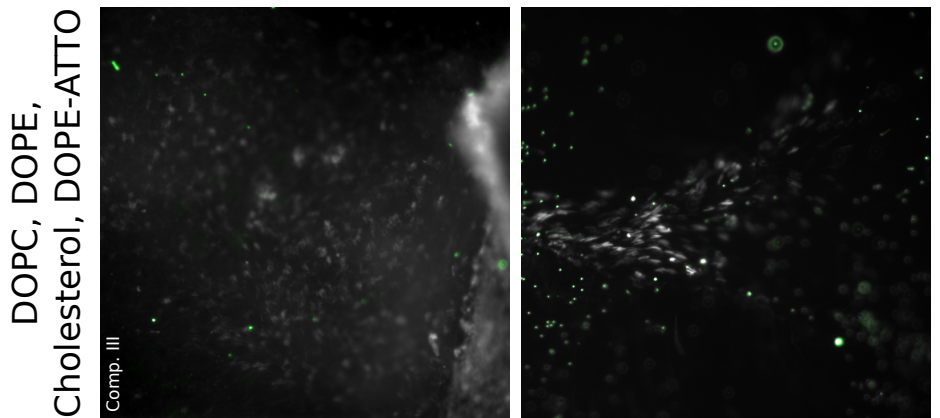

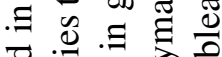

항 공

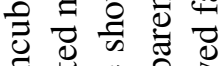

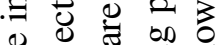

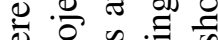

उ

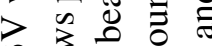

$>$ 司紊

\& क
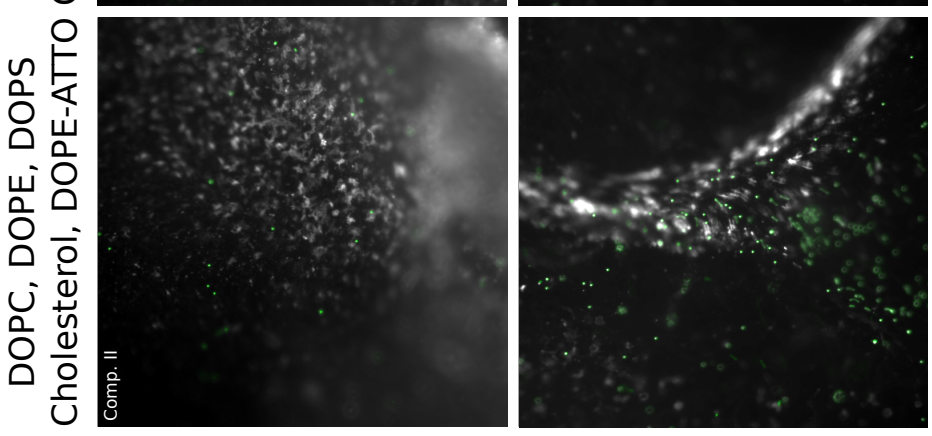

9 씨커

L MO키

些总导

츨 응 ส

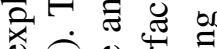

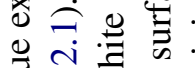

क

$\because$ 记 $\Xi 0$

$\ddot{\square} 5$ 范

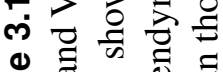

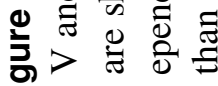




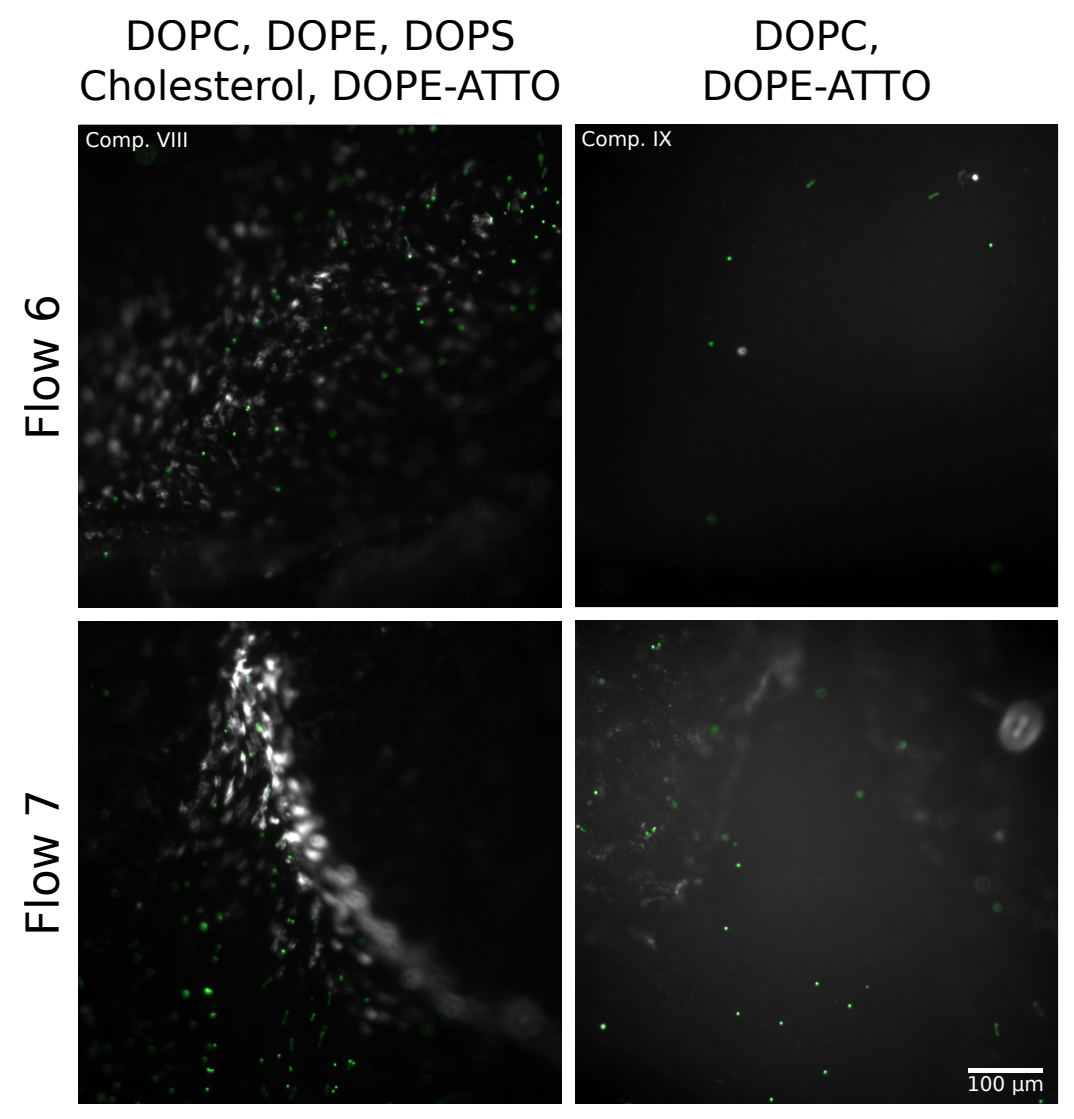

Figure 3.15: Tissue explants of the v3V were incubated in diluted fluorescently-labeled liposome solution of lipid composition VIII and IX (Tab. 2.2). The figure shows projected movies taken in the region of flow 6 and flow 7 after the incubation process. Liposomes are shown in white and fluorescent beads are shown in green. Tissue explants incubated in a solution of liposomes of composition VIII which contains DOPC, DOPE, DOPE-ATTO, DOPS and cholesterol, show strong liposome attachment to ependymal cilia. After incubation in a solution of liposomes of composition IX which contains DOPC and DOPE-ATTO only, liposomes did not attach to ependymal cilia strongly. In case of both compositions liposome attachment to cells of the surrounding parenchyma was found. Scale bar $=100 \mu \mathrm{m}$. 


\subsubsection{Monitoring liposome-ependymal cilia interactions}

The interaction process between liposomes and cilia of the $\mathrm{v} 3 \mathrm{~V}$ was monitored by imaging beating cilia bundles in the presence of freshly added fluorescently-labeled liposomes using DIC and fluorescence microscopy simultaneously (Sec. 2.6.2). The transport and interaction process was observed from top view using the open pouch preparation (Sec. 2.4.1) and from a lateral aspect after cutting the $\mathrm{v} 3 \mathrm{~V}$ tissue into thin slices (Sec. 2.6.2 and 2.7.2, several slices per animal were used $n=3$ App. Tab. 5.2). All experiments were performed at room temperature. These experiments were performed in collaboration with the lab of Dr. Cecilia Lo of the University of Pittsburgh.

Depending on the region of the $\mathrm{v} 3 \mathrm{~V}$, liposomes were transported in the cilia-induced flow towards the ependymal cell layer and entered the near wall flow or were transported away from the ependymal cell layer out of the near wall flow. Liposomes within the near wall flow were transported closely along the ependymal cells, where some of them came into contact and even could attach to ependymal cell cilia. Images of a representative movies are shown in Fig. 3.16 (Movie M3). In Fig. 3.16 A liposome transport within the near wall flow is shown from top view. Single liposomes are shown to get into contact with ependymal cell cilia and are either transported farther (Fig. 3.16 A black arrow) or attach to ependymal cilia (Fig. 3.16 $\mathbf{A}$ white arrow).

Fig. 3.16 B shows a single liposome transported towards the ependymal cell layer from afar and getting into contact with the ependymal cilia. Additionally, ependymal cilia bundles, with fluorescently-labeled liposomes attached, were monitored over time. An example is shown in Fig. 3.16 C. However, the position of the fluorescent signal did not change over time. These results support the previous findings of liposome propagation along the ciliary prescribed streamlines after local application into the near wall flow.

\subsubsection{Localization and analysis of liposome-ependymal cilia attachment mechanism}

Live imaging of beating cilia bundles after incubation of $\mathrm{v} 3 \mathrm{~V}$ explant in a solution with fluorescently-labeled liposomes and application of such liposomes into the cilia-generated near wall flow revealed, that liposomes attach to ependymal cells, predominately to ependymal cilia bundles. The strongest attachment site seemed to be closer to the cilia tips compared with the base of the cilia and the ependymal cell surface. However, these experiments do not show whether liposomes are just adsorbed to the cilia membrane and 
cell surface or taken up by the cell. To begin to investigate this, v3V tissue was fixed after contact with fluorescently-labeled liposomes and analyzed by confocal microscopy.

V3V explant was incubated in 1:500 diluted, homogeneous liposome solution, consisting of liposome of composition II (Tab. 2.1) prepared by size exclusion chromatography. The incubation time was $30 \mathrm{~min}$ at RT. After documenting the liposome attachment over the whole $\mathrm{v} 3 \mathrm{~V}$ area, the explant was fixed with paraformaldehyde and stained with DAPI, to visualize the nuclei of the cells, and TRITC-phalloidin, to stain filamentous actin. As negative control a $\mathrm{v} 3 \mathrm{~V}$ explant was incubated in DMEM for $30 \mathrm{~min}$ and processed under the same conditions. Afterwards, the inflow region and the regions of flow 6 and flow 7 were imaged using confocal microscopy. Fig. 3.17 shows representative projected and merged images of the recorded confocal stacks.

Small spots were visible on the v3V ependymal cells, following the incubation of the explant in diluted, fluorescently-labeled liposome solution. In the inflow region, at the head of flow 1, flow 2 and flow 3, liposomes were attached equally over the whole area (Fig. 3.17). In the regions of flow 6 and flow 7 the attached liposomes accumulate in certain locations, which seems to correlate with individual cells. Thin, hair-like structures, which emerge from the ependymal cell surface, are discernible in flow 7 due to the liposome attachment (Fig. 3.17 green arrow). These structures are very likely to represent ependymal cilia bundles. This is consistent with the results of the live imaging experiments. Based on the fluorescence intensity, the strongest liposome attachment resides at the tip of these structures, which is consistent with the recording of beating cilia bundles with attached liposomes (Fig. 3.12).

Next, fluorescently-labeled liposomes of composition IV (Tab. 2.1) prepared by size exclusion chromatography, were applied locally to the cilia-generated near wall flow of flow 6 and flow 7 of a v3V explant at RT. Additionally, a v3V explant was incubated in a 1:500 diluted liposome solution of the same composition for $10 \mathrm{~min}$. After recording short movies of the liposome attachment across the whole v3V area, the tissue was fixed and stained as described above. Confocal microscopy was used to image the position of liposomes on the fixed sample. Representative projected images of the movies, which show the liposome location directly after their local application or incubation and projected images of confocal stacks, are shown in Fig. 3.18.

The applied liposomes were transported along the cilia-generated streamlines in the region of flow 6 and flow 7. Along the propagation path, liposomes attached to beating cilia bundles and short movies of the process were recorded. Projections of movies taken 
after the attachment are shown in Fig. 3.18 A on the left side. After fixation and staining, the confocal images clearly showed numerous single spots in the same locations where liposomes attached themselves to the beating cilia bundles (Fig. $3.18 \mathrm{~A}$ right side and framed aera $\mathbf{a}$ on the left side). Further, these locations can be associated with individual cells underneath. Interestingly, only some ependymal cilia bundles along the propagation path show liposome attachment.

A similar observation was made after incubation of a v3V explant in a homogeneous liposome solution (Fig. 3.18 B). The sites of strong liposome attachment in the region of flow 6 and flow 7 were recorded directly after the incubation and projections of these are shown on the left side of Fig. 3.18 B. Projections of confocal stacks taken after fixation and staining of the same tissue are shown on the right side. The position of attached liposomes after the fixation and staining remained the same (Fig. $3.18 \mathbf{B}$ framed area b). Liposomes attached strongly to ependymal cilia bundles, visualizing them and allow for a clear identification. Although the whole area of $\mathrm{v} 3 \mathrm{~V}$ explant was equally exposed to liposomes, not all cilia bundles in the region of flow 6 and flow 7 showed the same level of liposomes attachment (Fig. $3.18 \mathbf{B}$ right side).

Studies of the interaction between liposomes and cells have shown that the main interactions are simple adsorption and endocytosis, which can follow the adsorption [10, 73, 98]. The adsorption process of liposomes to cells can result from specific interactions with cell-surface components [10,73] or electrostatic forces [10]. Other possible interactions with the cells plasma membrane are the fusion of liposomes or the exchange of liposome membrane components with it. [10, 102].

The results presented in Fig. 3.17 show small, individual spots on v3V ependymal cells, including the cells surface (Fig. 3.17 inflow) and cilia bundles (Fig. 3.17 flow 6 and flow 7). The same was found after the application of small amounts of liposomes to the near wall flow, as shown in Fig. 3.18. These findings suggest, that liposomes and ependymal cells interact through an adsorption process rather than fusion or the exchange of membrane components, because the latter two would lead to a spreading of the fluorescent dye over the whole ependymal cell membrane. It is not entirely clear which components of the liposomal membranes cause the interaction. However, liposomes used in these experiments were prepared from composition II and IV (Tab. 2.1) and both contain cholate as well as the lipid DOPS, which has a negatively charged head group. Additionally, composition II contained cholesterol. All three components could be involved in the adsorption process. 
The regional difference in liposome attachment to the ependymal cells indicate a higher affinity between liposomes and ependymal cells in certain regions of the $\mathrm{v} 3 \mathrm{~V}$, predominantly to ependymal cilia. This result supports the hypotheses of a more specific interaction between liposomes and the ependymal cells. Further, the local application of a small amount of liposome to the cilia-generated near wall flow revealed that liposomes only attach to the ependymal cilia bundles along the propagation path. This emphasizes the importance of precise cargo transport along the ventricular walls. 


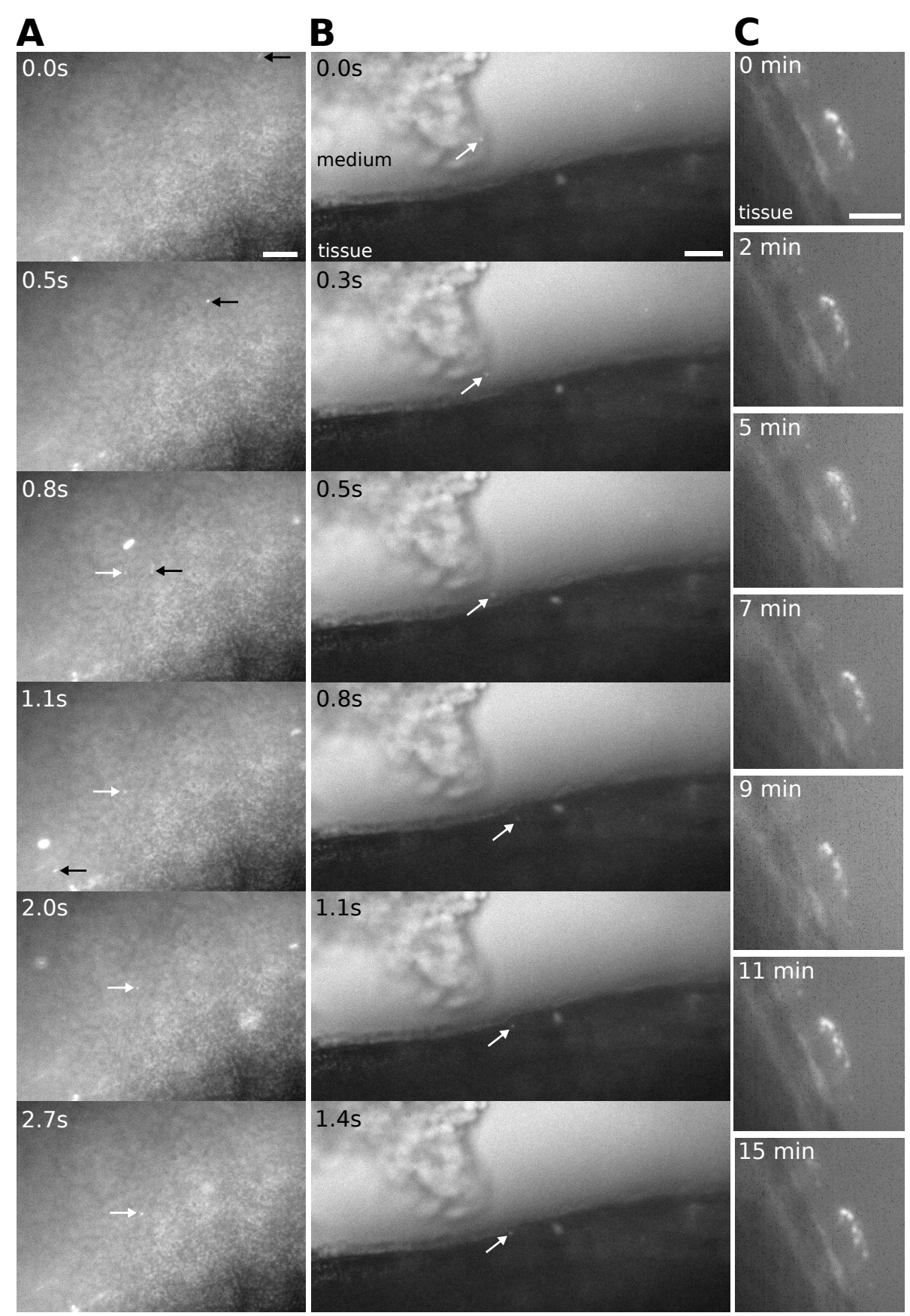

Figure 3.16: Interaction of fluorescently-labeled liposomes (white) with v3V ependymal cilia. Liposomes were prepared by size exclusion chromatography from stock solution of composition I (Tab. 2.1). A Liposomes were added to v3V explant and recorded from top view. Black arrow indicates the position of a liposome transported in the cilia-generated neat wall flow. White arrow indicated a liposome attached to a ependymal cilium. Scale bar $=20 \mu \mathrm{m}$. B V3V explant was cut into thin slices and cilia beating recorded from a lateral aspect. Pictures show the transport of liposomes over time. The white arrow indicates the position of a liposome that is transported towards the ependymal surface and gets attached with ependymal cilia. Scale bar = $20 \mu \mathrm{m}$. C Liposomes attached to ependymal cilia were recorded over $15 \mathrm{~min}$. The position of the attached liposomes does not change during the observed time period. Scale bar $=12 \mu \mathrm{m}$. 


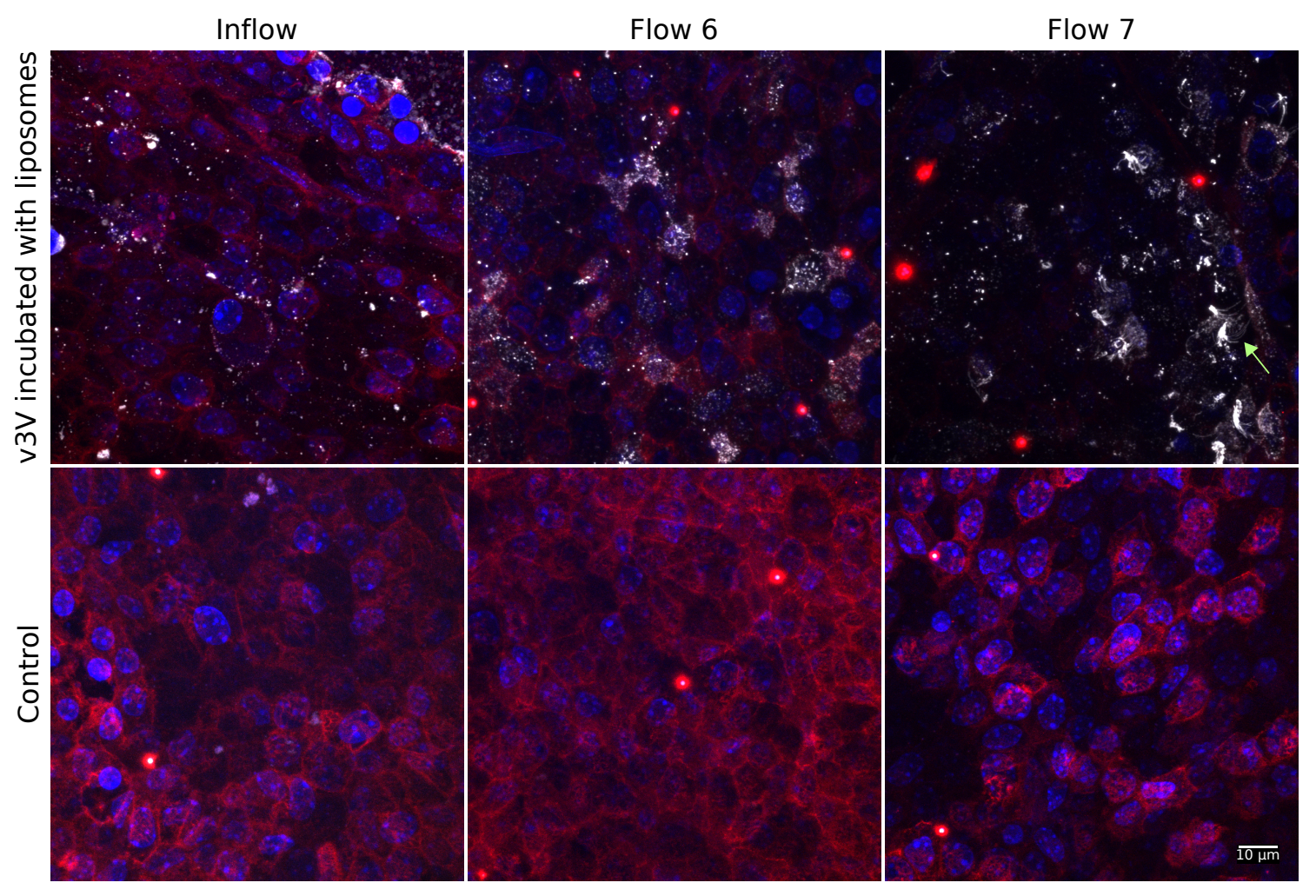

Figure 3.17: The v3V was incubated in diluted fluorescently-labeled liposome (white) solution (composition II), fixed and stained with DAPI (blue) and TRITC-phalloidin (red). As control a v3V sample was incubated in DMEM. The images show projections of confocal stacks taken in the regions of the inflow, flow 6 and flow 7. Bright red spots are fluorescent beads attached to the ependymal surface during the recording of the cilia-generated flow. After incubation, the fluorescent signal of liposomes is discreetly distributed on the ependymal cell surface. This suggests an attachment of liposomes to the ependymal cell rather than fusion with the cell membrane. The attachment to some cells in flow 6 and flow 7 is stronger in comparison to the remaining v3V ependymal surface. Green arrow indicates fine hair-like structures with liposome attachment. Scale bar $=10 \mu \mathrm{m}$. 


\subsection{Liposome interaction with ependymal cilia}

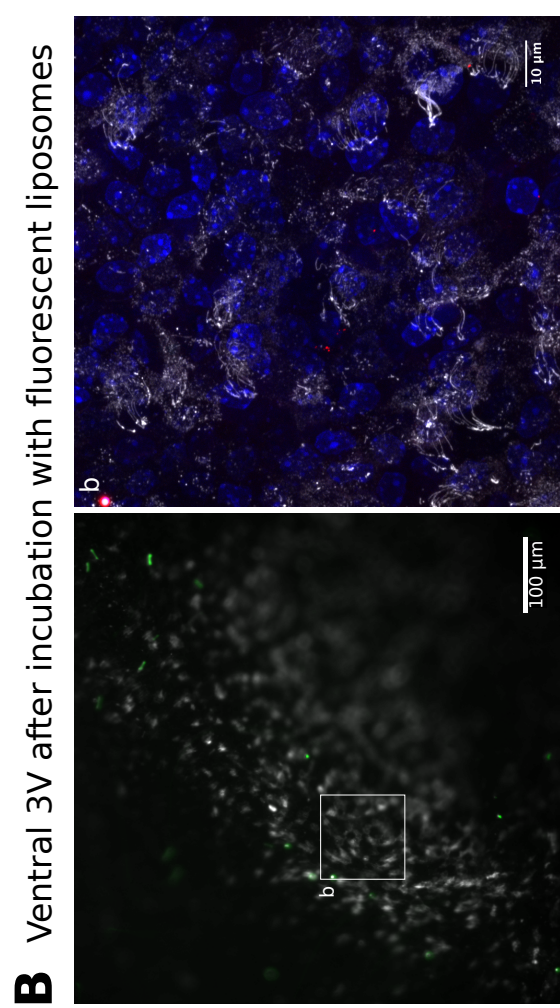

$9 \mathrm{MO}$ 이

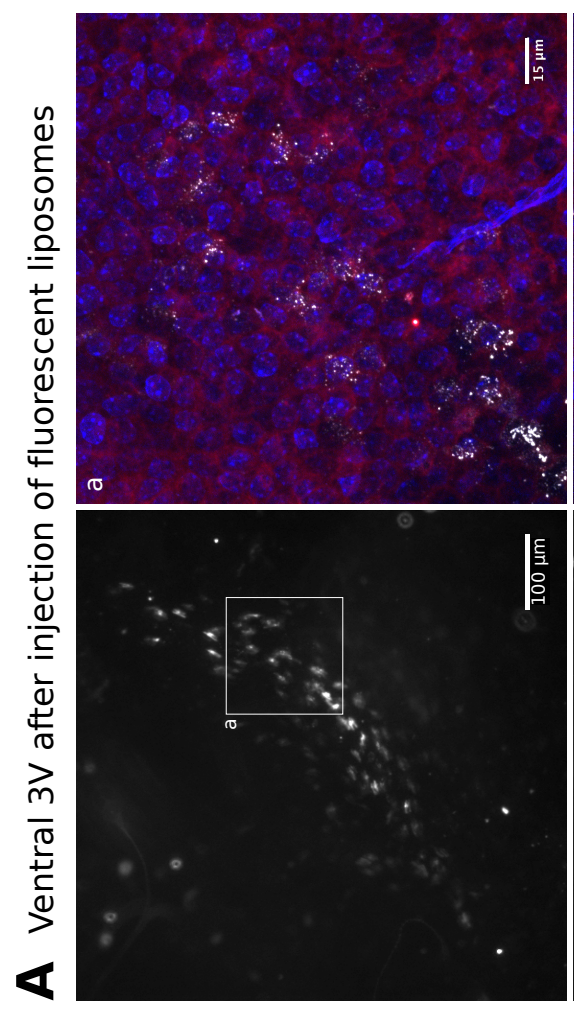

9 M이버

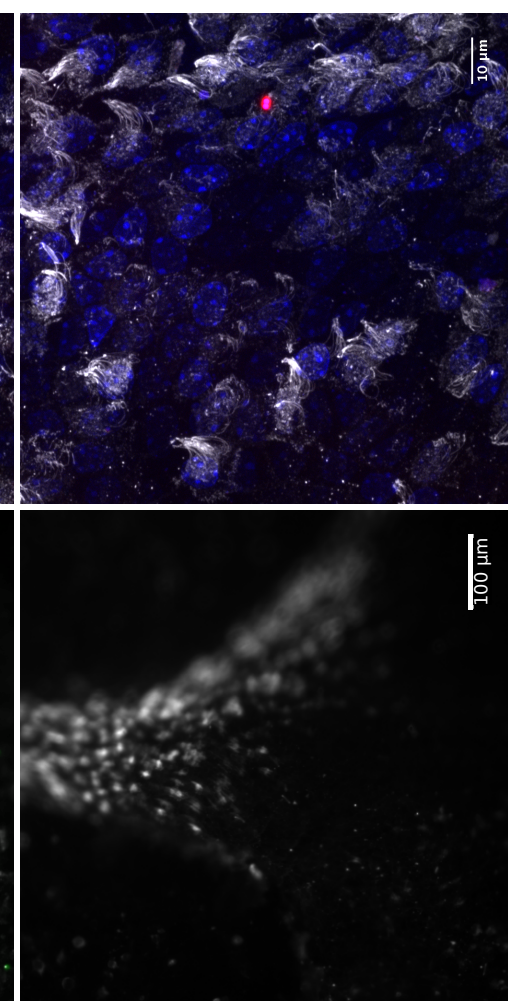

$\angle M$ M이

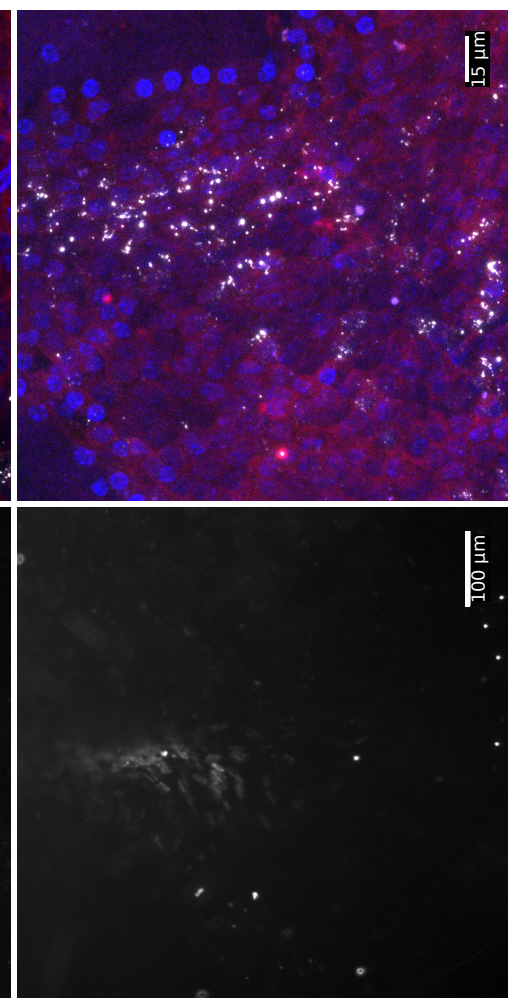

$\angle M O M$

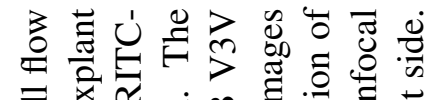

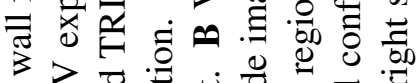

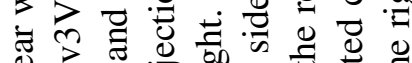

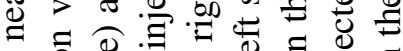

ช

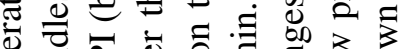

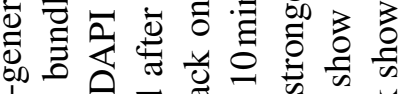

$\theta$ 的

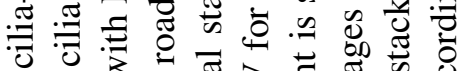

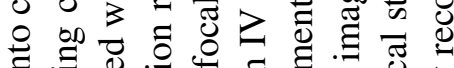

.

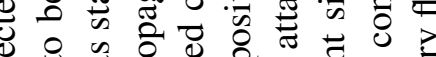

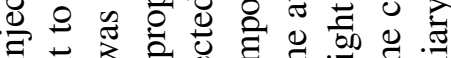

ज च

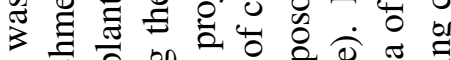

$\geq$ 可

䒕>

의의 0 항

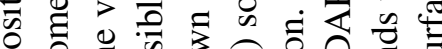

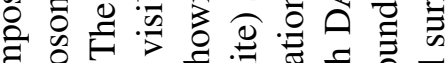

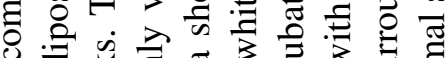

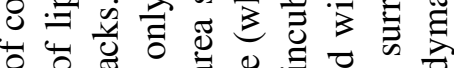

(1) 0 空

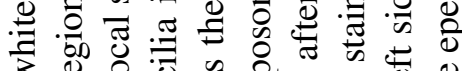

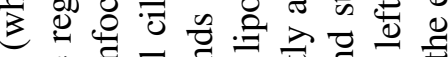

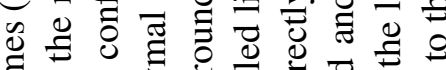

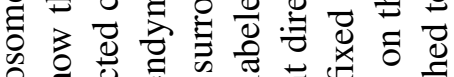

论

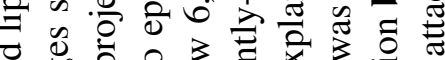

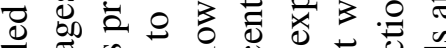

ष

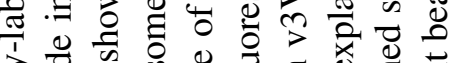

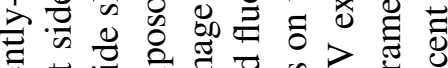

एँ

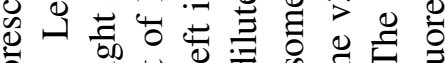

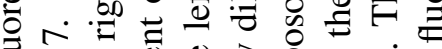

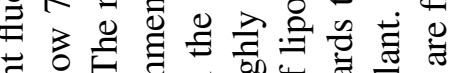

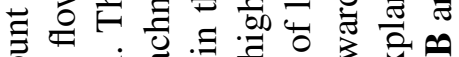

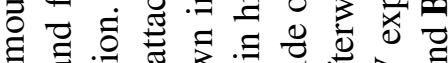

ब च

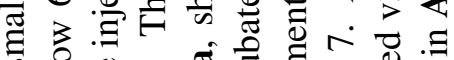

के

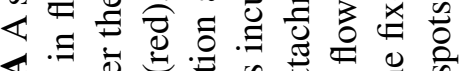

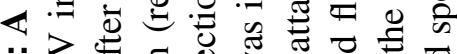

ö

ம் m 


\subsubsection{Concentration and time dependence of liposome attachment}

The local application of fluorescently-labeled liposomes in the v3V cilia-generated near wall flow revealed a region-dependent interaction between the transported liposomes and the ependymal cells. In the region of flow 6 and flow 7 liposomes attached to the ependymal cilia bundles within seconds. In contrast, liposomes transported along flow 1 and flow 2 just seem to be washed over the ependymal cells without indication of a strong affinity towards the ependymal cilia. For a more precise analysis, the attachment of liposomes to ependymal cells as a whole was distinguished between liposome attachment to ependymal cilia and the remaining ependymal cell membrane (hereinafter also called cell surface).

The incubation of v3V explants in homogeneous, fluorescently-labeled liposome solution for several minutes demonstrated that the affinity of liposome attachment in the region of flow 6 and flow 7 is indeed strongest. But a very faint attachment on ependymal cells was also found in other regions of the v3V. However, it was not clear if liposome attachment in these regions was preferential to the ependymal cilia or the ependymal cell surface. This observation indicates that under a constant supply over time, a larger number of liposomes may also attach in regions other than the region of flow 6 and 7 . To begin to investigate the kinetic aspect of the liposome and ependymal cell interaction over the entire v3V area, explants of the v3V were incubated in solutions of varying liposome concentrations for different incubation times.

Explants of the v3V were incubated in 1:10, 1:250 and 1:500 diluted liposome solution for $10 \mathrm{~min}, 30 \mathrm{~min}$ and $1 \mathrm{~h}$ at RT. Afterwards, the attachment of liposomes to ependymal cilia and the ependymal cell surface was imaged in several regions of the $\mathrm{v} 3 \mathrm{~V}$. The amount of liposome attachment was monitored in detail in the inflow region, at the head of flow 1, flow 2 and flow 3 (Fig. 3.19), in the separatrix region, where flow 3 and flow 4 are opposing each other (Fig. 3.20), and in the distributor region at the head of flow 6 and flow 7 (Fig. 3.21). Several movies in different focal planes were taken in each region. Then the movies were projected using Fiji and stitched together for each region, in a way that the ependymal cell surface and beating cilia bundles were in focus. Liposomes attached to the ependymal cell surface were visible as stationary fluorescent spots in the intensity projection images. Liposomes attached to beating ependymal cilia showed as thin lines in the intensity projection images.

Overall, an increase of the incubation time led to an increase of liposomes attached 
to ependymal cells. The same was observed for an increase in concentration. The site of attachment on the ependymal cells seems to differ across the regions in the v3V. In the inflow region (Fig. 3.19) the number of small, stationary fluorescent spots on the ependymal cell surface increases from the lowest concentration and shortest incubation time (1:500 dilution, $10 \mathrm{~min})$ to the highest concentration and longest incubation time (1:10 dilution, $1 \mathrm{~h}$ ). However, liposomes attached to beating cilia bundles were recognizable at the longest incubation time in incubations with a 1:500 and 1:250 dilution. The liposome-cilia attachment was clearly visible at $30 \mathrm{~min}$ incubation time and 1:10 dilution. After incubation in a 1:10 diluted liposome solution for $1 \mathrm{~h}$, the ependymal surface was densely covered with attached liposomes. This could imply that the fluorescently-labeled liposomes predominantly stick to the ependymal cell surface in the inflow region.

In the separatrix region, the attachment of liposomes to ependymal cilia is clearly visible (Fig. 3.20) and strong in comparison to the attachment to the ependymal cell surface. Again, after $1 \mathrm{~h}$ incubation in a 1:10 diluted liposome solution the ependymal cells are densely covered with fluorescently-labeled liposomes, but the attachment to the ependymal cell surface seems to be stronger. In the region of the distributor, at the head of flow 6 and flow 7, the attachment of liposomes to the ependymal cell cilia and surface seems to increase in an equal amount over time and with increasing concentration (Fig. 3.21).

The increasing attachment of liposomes to the ependymal cells in all regions over time and with higher liposome concentration continued until ependymal cells were tightly covered with fluorescently-labeled liposomes. The result could imply that, while under constant supply of liposomes, the process of liposome attachment to ependymal cells over time reaches a saturated state. The incubation in a highly diluted liposome solution (1:500 dilution) revealed, that even after $30 \mathrm{~min}$ incubation, the ependymal cells of the monitored regions showed proportionally little liposome attachment. This result indicates, that up to a certain amount of liposomes over time the cilia-generated flow effectively transports liposomes over the ependymal cells. 


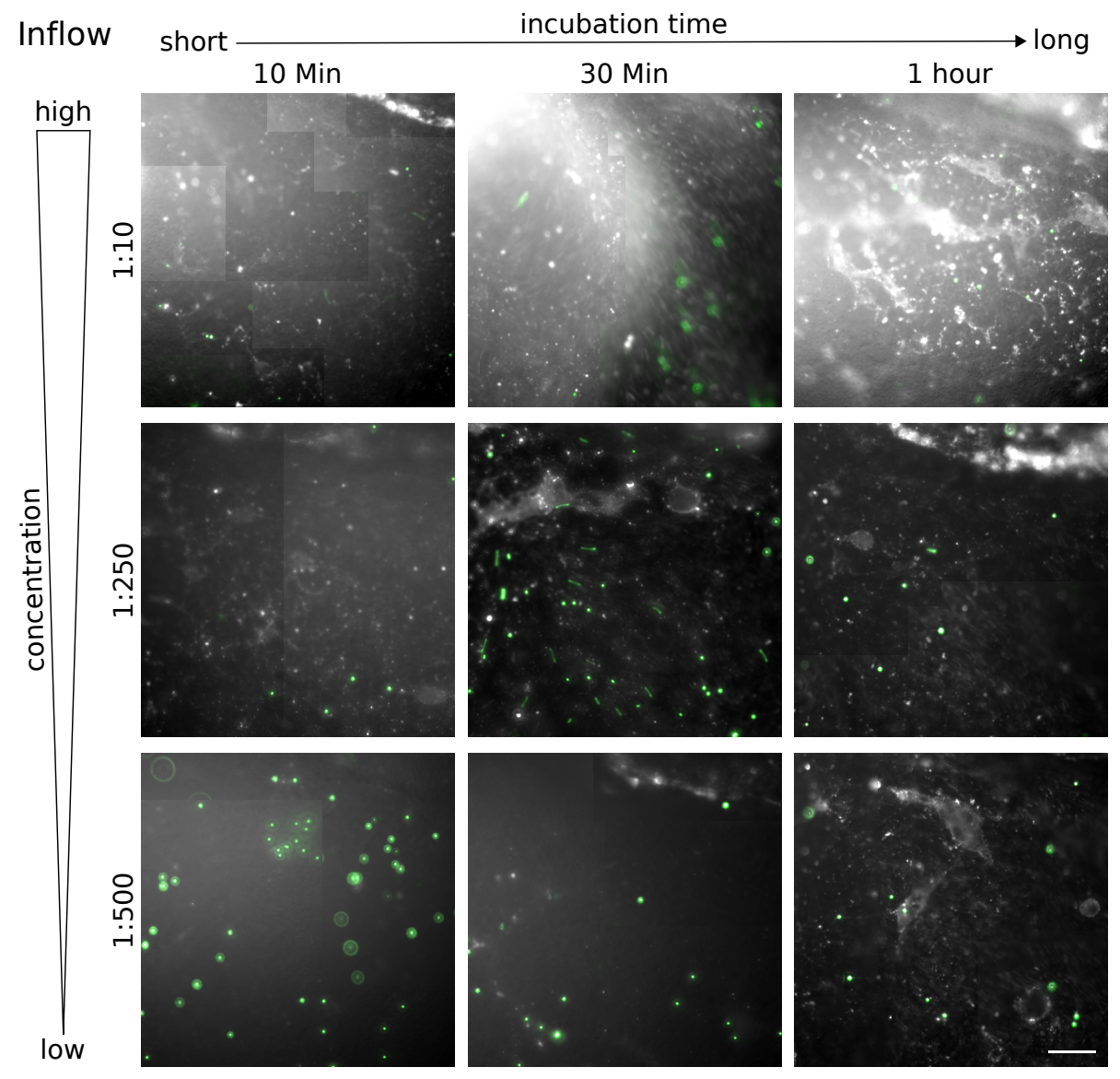

Figure 3.19: V3V explants were incubated in homogeneous fluorescently-labeled liposome solution in a dilution ratio of 1:10, 1:250 and 1:500 for $10 \mathrm{~min}, 30 \mathrm{~min}$ and $1 \mathrm{~h}$. Liposomes were prepared by size exclusion chromatography of liposome stock solution of composition II. The panel shows the inflow region close to the anterior commissure after incubation. Fluorescent signal of liposomes is shown in white, fluorescent beads are shown in green. The attachment of liposomes to beating cilia as well as to the ependymal surface increases with increasing incubation time and decreased with higher dilution factor. Scale bar $=50 \mu \mathrm{m}$. 
3.2 Liposome interaction with ependymal cilia
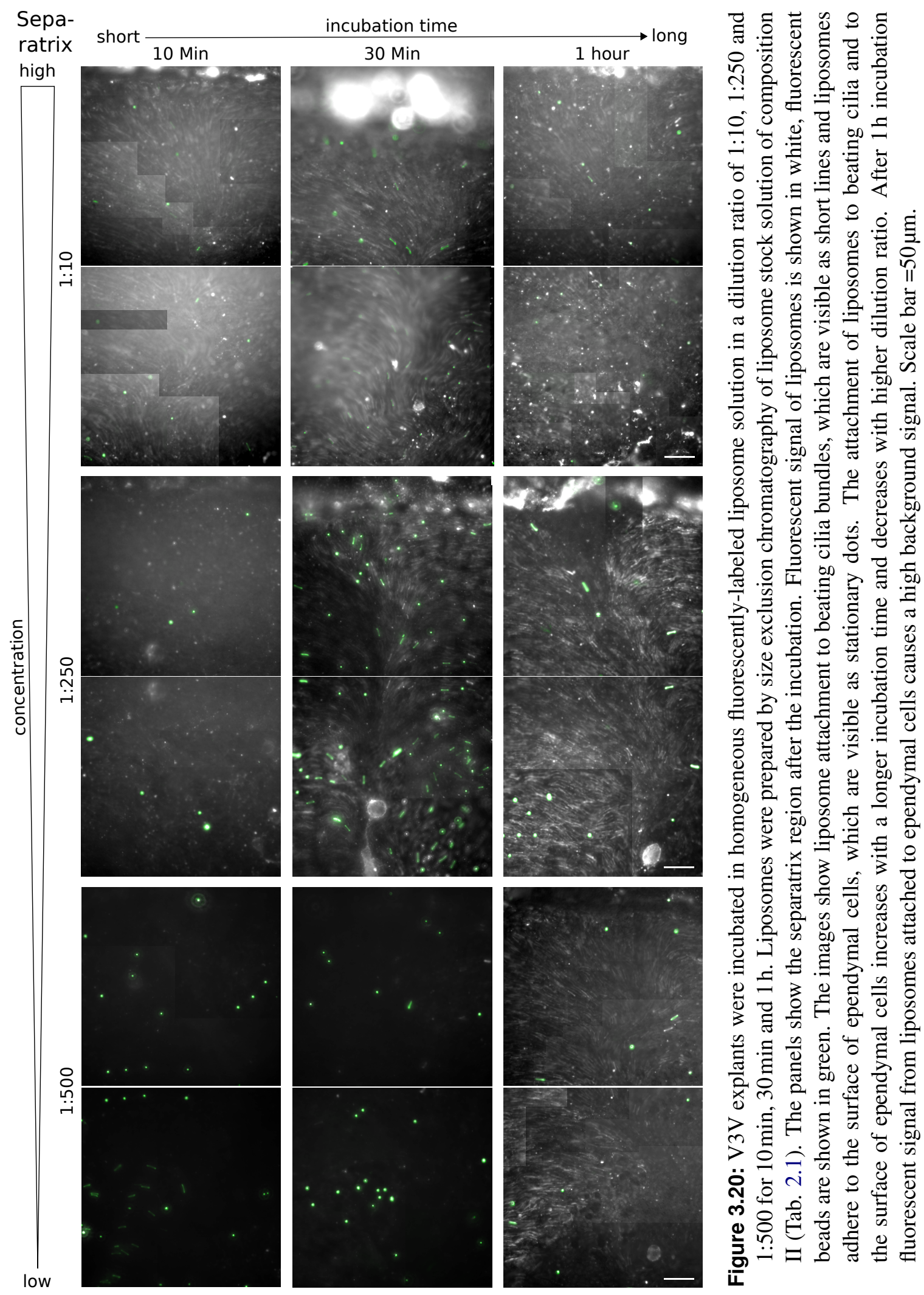


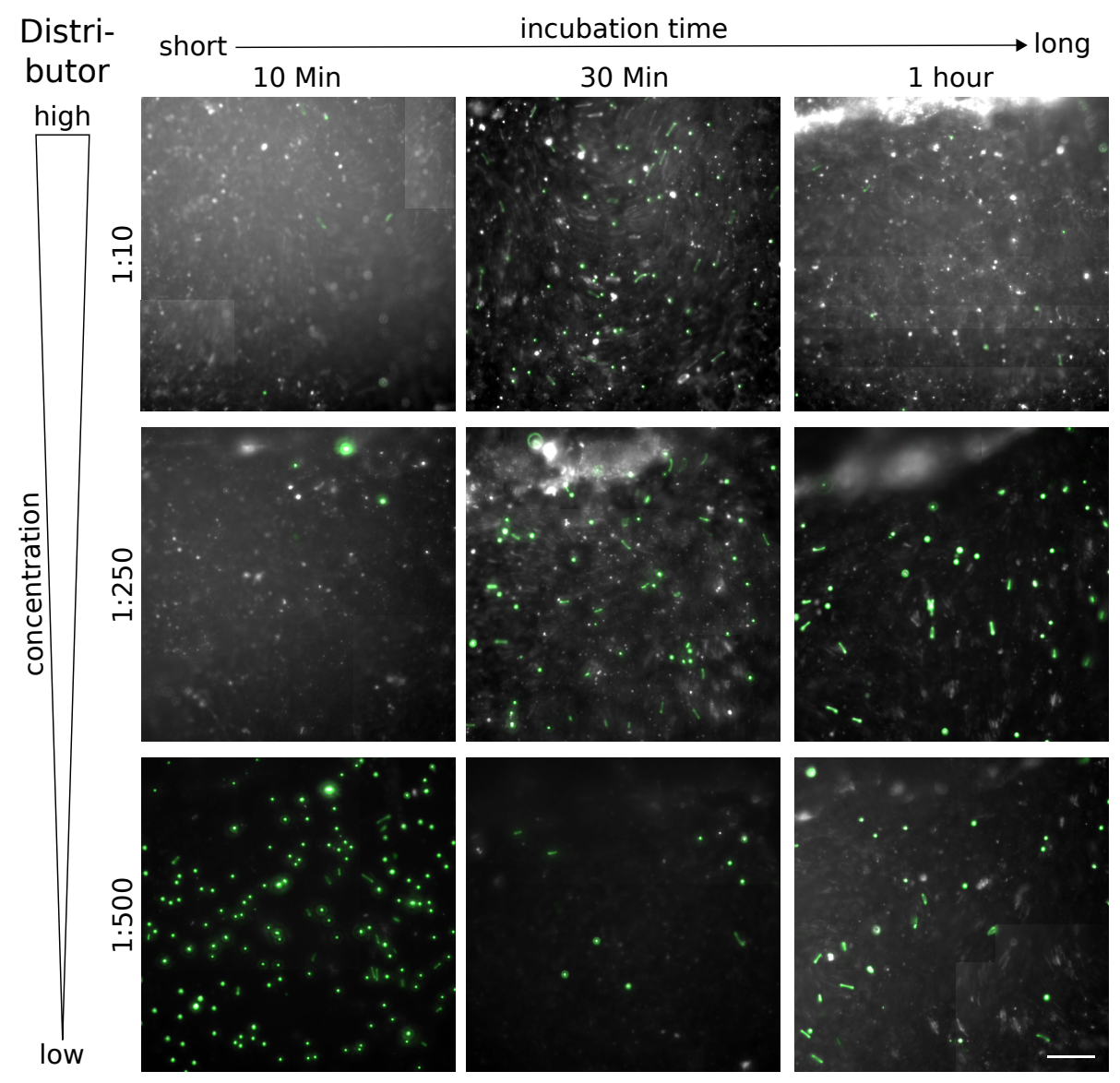

Figure 3.21: V3V explants were incubated in homogeneous fluorescently-labeled liposome solution in a dilution ratio of 1:10, 1:250 and 1:500 for $10 \mathrm{~min}, 30 \mathrm{~min}$ and $1 \mathrm{~h}$. Liposomes were prepared by size exclusion chromatography of liposome stock solution of composition II. The panel shows the distributor region close to the posterior duct after incubation. Fluorescent signal of liposomes is shown in white, fluorescent beads are shown in green. The attachment of fluorescent liposomes to beating cilia increases over incubation time and decreases with higher dilution factor. At a dilution rate of 1:10 the background signal from liposomes attached to ependymal cells increases strongly over incubation time. Scale bar $=50 \mu \mathrm{m}$. 


\subsubsection{Interaction of liposomes with cilia and flagella}

The functions of cilia range from sensory to motile processes, depending on the origin of the cells they emerge from. The ciliary membrane protein and lipid composition is highly and specifically regulated and distinct from the plasma membrane [40, 97]. To investigate if cilia of other origins show a similar interaction, like the one observed between v3V ependymal cilia and liposomes, ciliated tissue of different origins and species were exposed to fluorescently-labeled liposomes. These experiments were performed in collaboration with the lab of Dr. Cecilia Lo of the University of Pittsburgh.

Mouse sperm cells were isolated and incubated at $37^{\circ} \mathrm{C}$ until use (Sec. 2.8.4, Tab. 5.2). The sperm cells were kept in sufficient DMEM all the time. Fluorescently-labeled liposomes of composition I (Tab. 2.1), prepared by size exclusion chromatography, were added to the sperm cells. It was observed, that liposomes were hit by sperm cells flagella, but, the liposomes did not attach (Movie M4). In contrast, the heads of motile sperm cells became fluorescent within a few minutes after fluorescently-labeled liposomes were added (Fig. 3.22 A; $n=3$, Tab. 5.2). After $1 \mathrm{~h}$ of incubation at RT most sperm cells were immobile, likely dead and strongly fluorescent (Fig. 3.22 B). These results indicate that liposomes of the used composition primarily interacted with the heads of the sperm cells and not with the flagella.

Human nasal epithelia cells were isolated from a healthy volunteer and purified (Sec. 2.8.5). The cells were transferred to a small chamber between two cover glasses. Fluorescently-labeled liposomes of composition I (Tab. 2.1), prepared by size exclusion chromatography, were added to the cells and the interaction between liposomes and beating cilia as well as the cell surface was recorded by confocal microscopy. Fig. 3.22 $\mathbf{C}$ shows a bright field images of human nasal epithelia cells and the maximum intensity projection of the recorded fluorescence signal. The beating cilia moved the surrounding medium and liposomes were transported towards the cell. Liposomes came into close contact with the beating cilia. For v3V cilia bundles we showed, that liposomes predominantly attach to the cilia tips. After the addition of fluorescent liposomes, human nasal epithelia cells presented a higher fluorescent intensity signal at the base of the cilia, close to the cell surface. Further, it was observed that liposomes were transferred towards the cell surface after contact with the beating cilia (Movie M5).

Fallopian tubes of CD1 wildtype mouse (Sec. 2.8.2, Tab. 5.2) were isolated, diluted fluorescently-labeld liposome solution (composition I, Tab. 2.1) was applied and their 
propagation recorded simultaneously with the beating cilia. A maximum intensity projection of a representative movie is shown in Fig 3.22 D. Liposomes were transported along the ciliary flow and came into contact with beating cilia bundles. However, even after a long incubation time liposome did not attach to cilia bundles or the cell surface. A similar observation was made after adding liposomes (of composition II, prepared by the same method) to fallopian tube tissue isolated from rabbits $(n=2$, Fig. $3.22 \mathbf{E}, \mathrm{Sec}$. 2.8.3). Although, the whole area of each isolated tissue was searched for liposome attachment, no interaction comparable to that one observed in regions of the $\mathrm{v} 3 \mathrm{~V}$ was found. Because the tissues examined did not include the whole ciliated area of the fallopian tube of mouse and rabbit, an interaction in other regions not being analyzed cannot be excluded completely.

Mouse trachea was isolated and cut into thin rings (Sec. 2.8.1). Afterwards, fluorescentlylabeled liposomes of composition I (Tab. 2.1), prepared by size exclusion chromatography, were added to the surrounding buffer. An interaction between liposomes and cilia bundles, was not observed for the examined areas. 

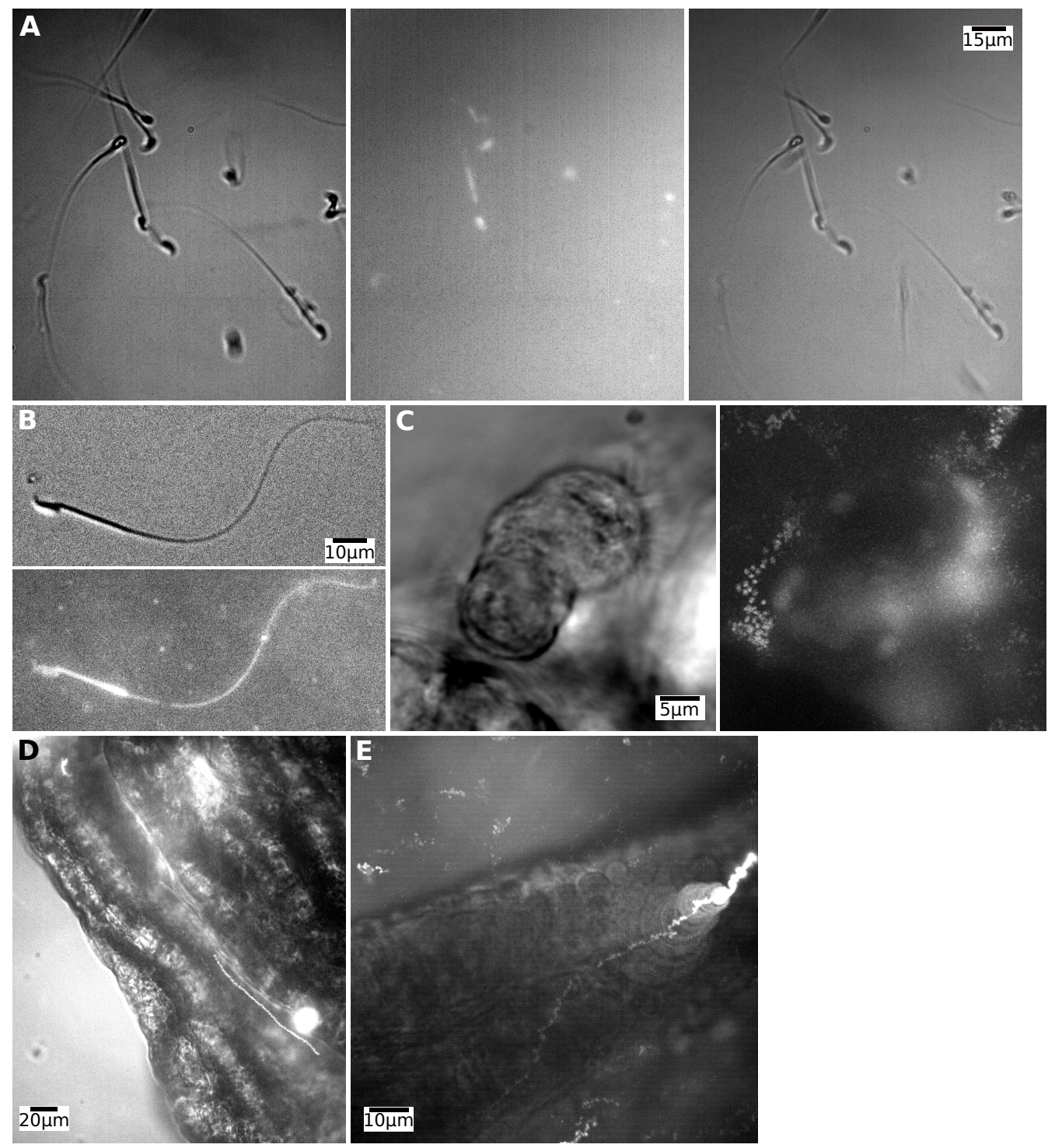

Figure 3.22: Fluorescently-labeled liposomes of composition I were added to ciliated cells and tissue, and sperm flagella. A Moving mouse sperm cells several minutes after adding fluorescently-labeled liposomes. First image $=$ DIC, second image $=$ fluorescence signal, third merged image of first and second picture. B Mouse sperm cell stuck to cover glass and not moving after $1 \mathrm{~h}$ incubation with fluorescent liposomes. Upper image shows DIC image, lower fluorescent signal. C Liposomes were added to human nasal epithelia cells with beating cilia. Fist image shows DIC recording, the second shows the projection of the fluorescent signal. Liposomes were transported close to the cilia and got stuck close to the cell surface. D Liposomes were added to mouse oviduct. Ciliary beating and fluorescent signal were recorded simultaneously over time. Movies were projected using maximum intensity projection. Liposomes were transported along the surface. E Liposomes were added to rabbit oviduct. Ciliary movement and fluorescent signal was recorded simultaneously and projected. Liposomes were transported along the surface. 


\subsection{Interaction of EVs produced by Z310 cells with ependymal cilia and flagella}

Liposomes were chosen to study the transport of EV-like cargo in the cilia-generated near wall flow of the $\mathrm{v} 3 \mathrm{~V}$, because each step of the preparation process can be controlled and large amounts can be produced. Additionally, their chemical composition can be changed according to the experimental requirements. Although, the mechanism of transport of liposomes and extracellular vesicles itself presumably is similar, the interaction with ependymal cell may primarily be influenced by the lipid and protein content. The lipid and protein composition of natural EVs, exosomes and ectosomes, varies with their cellular origin and the state of the cell [8]. A known main source of exosomes secretion into CSF within the ventricular system of the brain is the choroid plexus [3,5]. Based on this, the immortalized Z310 choroidal epithelia cell line from murine choroid plexus was chosen for a first attempt to investigate the interaction between $\mathrm{v} 3 \mathrm{~V}$ ependymal cells and exosomes.

\subsubsection{Characterization of EVs isolated from Z310 rat choroid plexus cells}

Extracellular vesicles were isolated from the supernatant of cultured Z310 rat choroid plexus cells by size exclusion chromatography $(\mathrm{SECH})$, precipitation using polyethylene glycol (PEG) and ultracentrifugation (UC) (Sec. 2.3.5). The morphology of EV samples isolated by each method was analysed by electron microscopy. Samples isolated by SECH predominantly showed predominantly small, single particles (Fig. 3.23 A). A similar result was found for EV samples isolated by PEG (Fig. 3.23 C). Ultracentrifugation also provided EVs but each sample contained large clusters (Fig. $3.23 \mathbf{E}$ ).

The size distribution of samples isolated by each method was measured by nanoparticle tracking analysis. Samples isolated by size exclusion chromatography consistently showed a main peak around $100 \mathrm{~nm}$ (Fig. 3.23 B), which corresponded to the expected size. PEG isolated samples included more smaller particles, as a result the size distribution's main peak was usually found in a range of $60 \mathrm{~nm}$ to $85 \mathrm{~nm}$ (Fig. $3.23 \mathrm{D}$ ). The main peak of samples isolated by ultracentrifugation was found around $170 \mathrm{~nm}$ (Fig. 3.23 F). While all samples size distributions included smaller fractions of larger particles, SECH showed the fewest and smallest fractions of larger particles and UC produced the most and largest. 
The isolated EVs were found positive for exosomal enriched protein-markers such as Alix (programmed cell death 6 interacting protein), Flot 2 (Flotillin-2) and CD63 (Fig. 3.23 G). Samples were also analyzed for expression of AGO 2 (argonaute-2), as a negative control. Additionally, qPCR analysis supported previous findings and showed no contamination of the EV samples with cellular RNA ( $\underline{\mathrm{AGO} 2}$ and Golga 2, Fig. 3.23 H).

Extracellular vesicles of Z310 cells were fluorescently-labeled after isolation using specific antibodies against exosomal markers present. Fig. $3.23 \mathbf{I}$ and $\mathbf{J}$ show representative immunofluorescence images of EVs labeled with antibodies against Alix and Flot 2.

To investigation the transport of exosomes along the cilia-generated near wall flow as well as their interaction with ependymal cells, EVs that are not affected by the isolation method are required. Ultracentrifugation produced large clusters within the sample and therefore was excluded as suitable isolation method. The cleanest EV samples for mRNA analysis were obtained by PEG, yet at times electron microscopy revealed residual material of the isolation process. Therefore, EVs isolated by SECH were mainly used for experiments with $\mathrm{v} 3 \mathrm{~V}$ explants.

The isolation and characterization of EVs was performed by Dr. Zuzana Ditte (MaxPlanck Institute for Biophysical Chemistry). The mRNA expression analysis (Fig. 3.23 H) was performed by Dr. Tamara Rabe (Max-Planck Institute for Biophysical Chemistry).

\subsubsection{Transport and interaction of Z310 EVs with v3V ependymal cells}

\section{Live imaging of Z310 EVs interaction with v3V ependymal cilia}

To study the interaction between extracellular vesicles and ependymal cilia bundles of the $\mathrm{v} 3 \mathrm{~V}$, the transport of fluorescently-labeled EVs was observed using DIC and fluorescence microscopy. The v3V explant was cut into thin slices, in order to observe the ciliary beating from a lateral view (Sec. 2.7.2). Extracellular vesicles from Z310 rat choroid plexus cells were added to the surrounding medium of the v3V slices. Several slices per $\mathrm{v} 3 \mathrm{~V}$ were used ( $n=3$, Tab. 5.2). The transport of EVs and beating of ependymal cilia bundles was recorded simultaneously. This experiment was performed in collaboration with the lab of Dr. Cecilia Lo of the University of Pittsburgh.

Depending on the monitored region of the $\mathrm{v} 3 \mathrm{~V}$, EVs were transported along the near wall flow very close to the beating ependymal cilia (Fig. 3.24 A, Movie M6) or even made contact with the ependymal cilia (Fig. 3.24 B, Movie M7). In other regions EVs were kept away from closer contact with the ependymal cells. The observed transport be- 

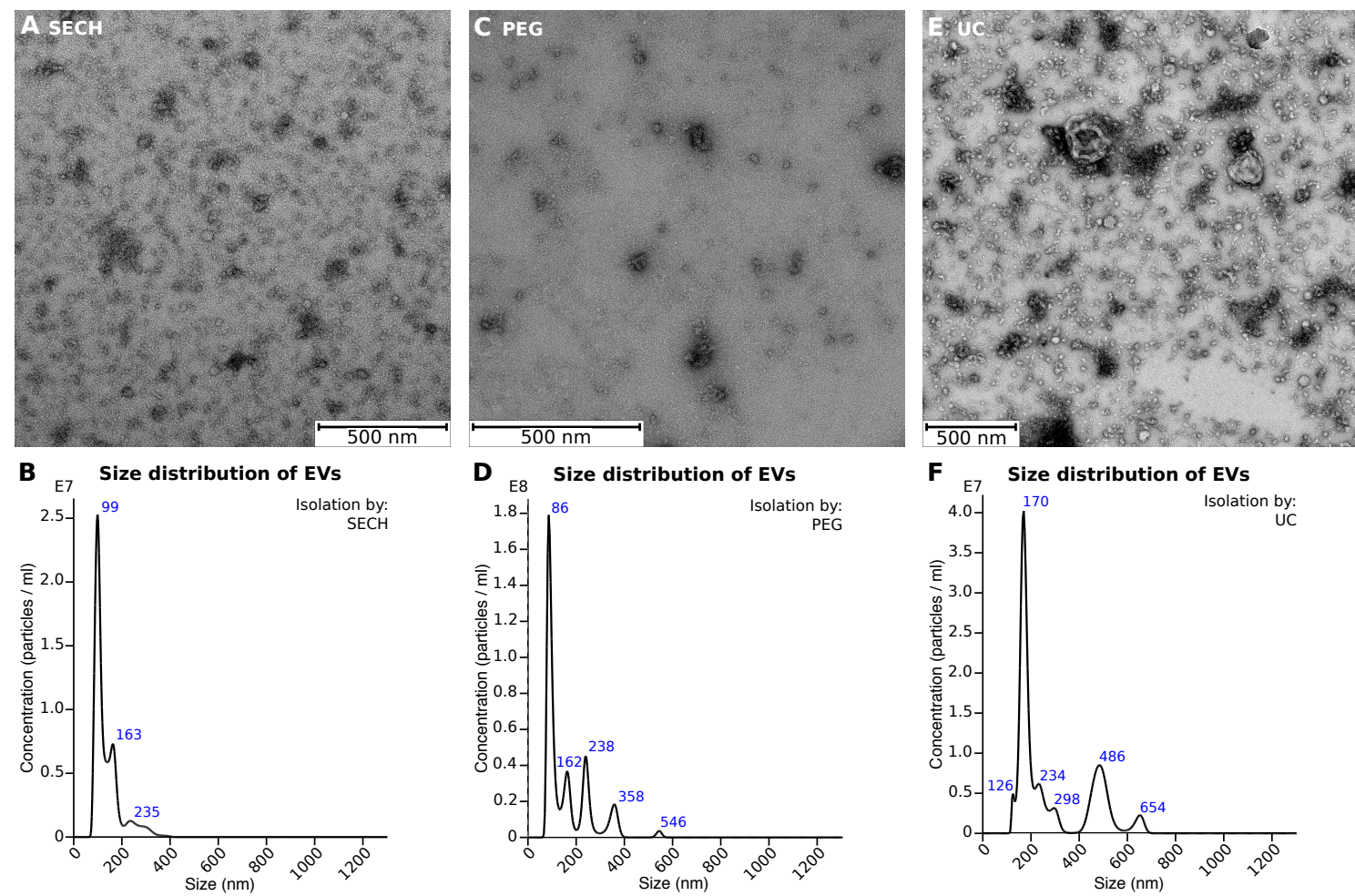

F Size distribution of EVs
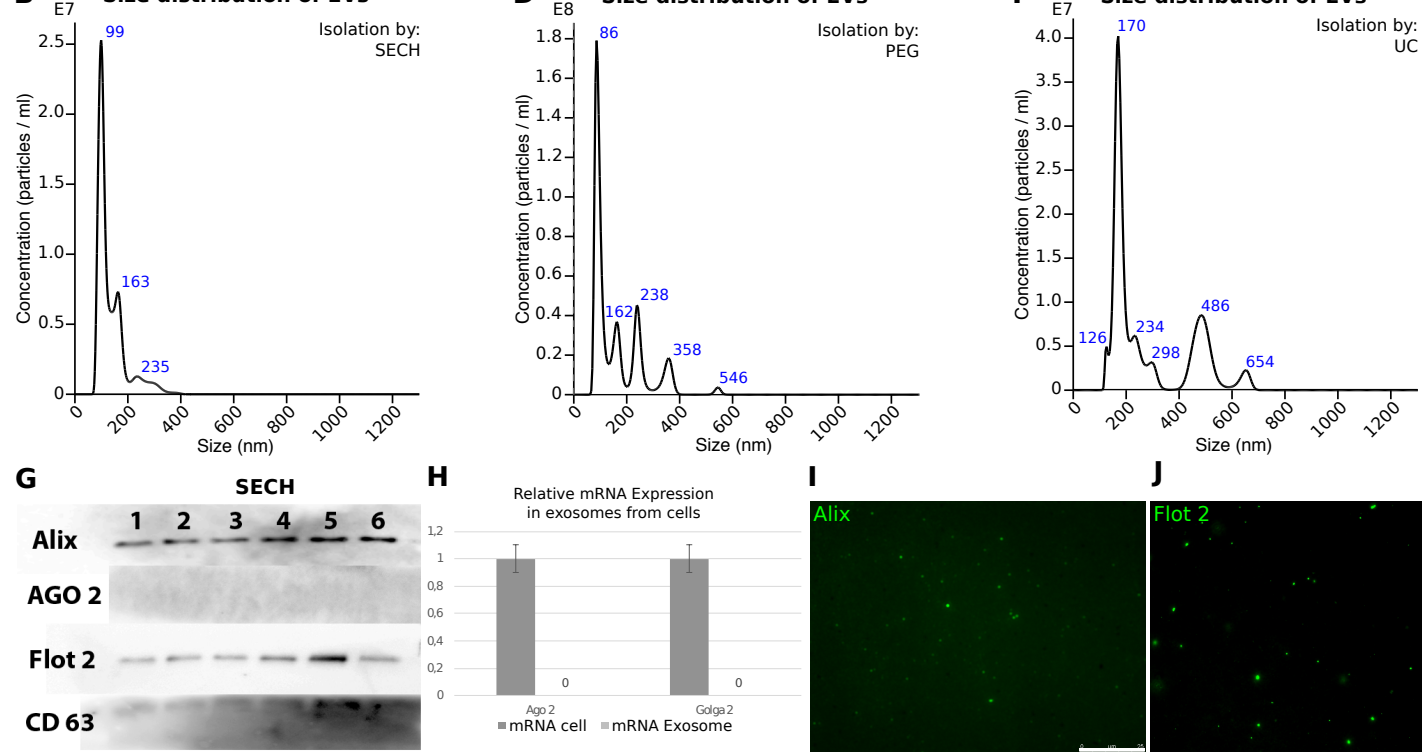

J

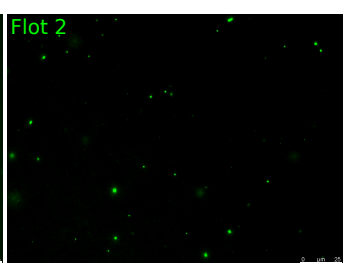

Figure 3.23: EVs were isolated from $\mathrm{Z} 310$ supernatant by size exclusion chromatography (SECH), by precipitation using polyethylene glycol (PEG) and ultracentrifugation (UC). A, $\mathbf{C}$ and $\mathbf{E}$ show representative electron microscopy images from EVs samples. Below, $\mathbf{B}, \mathbf{D}$ and F show representative size distributions of EVs samples measured by NTA (dilution 1:10). G representative western blott of Z310 EVs isolated by size exclusion chromatography. The fractions were analyzed for the markers CD36, Alix and Flotillin-2. AGO2 was used as negative control. $\mathbf{H}$ shows the mRNA expression within Z310 cells and Z310 cell produced EVs. I and $\mathbf{J}$ show representative immunofluorescent images of EVs after labeling with Alix and Flotellin-2 (scale bar $=25 \mu \mathrm{m}$ ).

haviour of EVs was similar to the recorded transport of liposomes (see Sec. 3.2.3). These results support, the hypotheses that liposomes and EVs show similar transport properties. Further they suggest, that the contact between extracellular vesicles and ependymal cell is possible. 
A

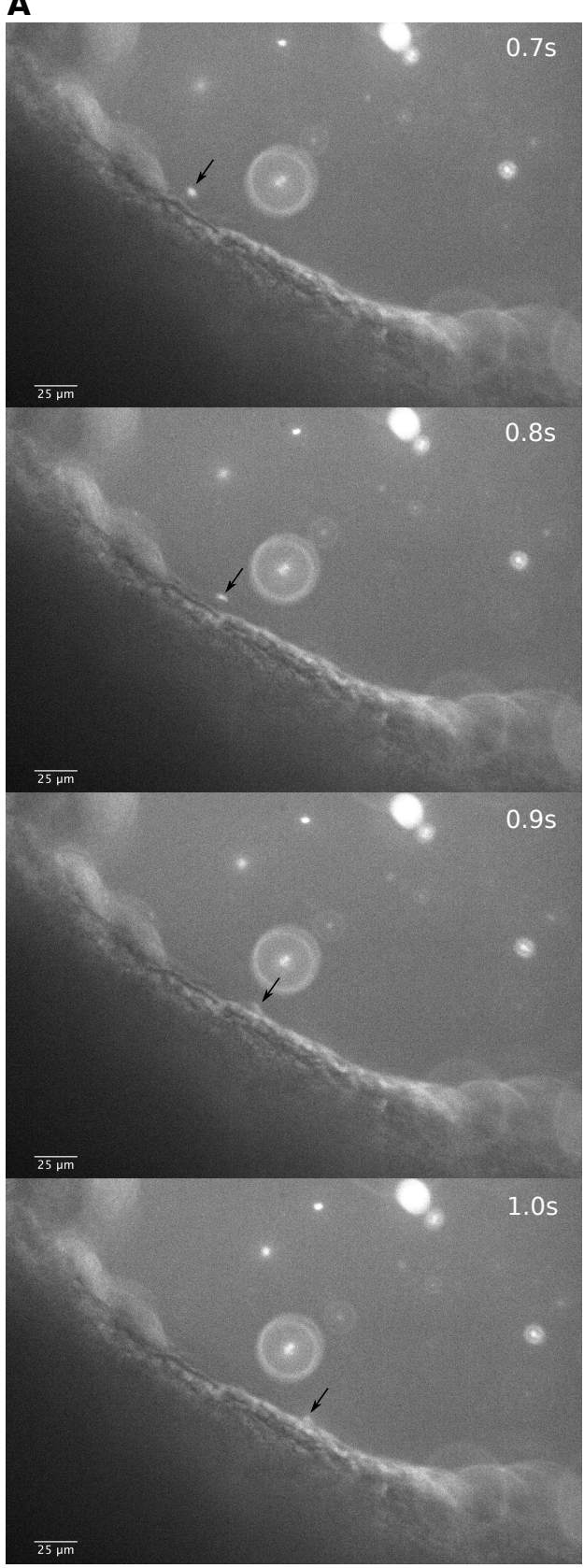

B

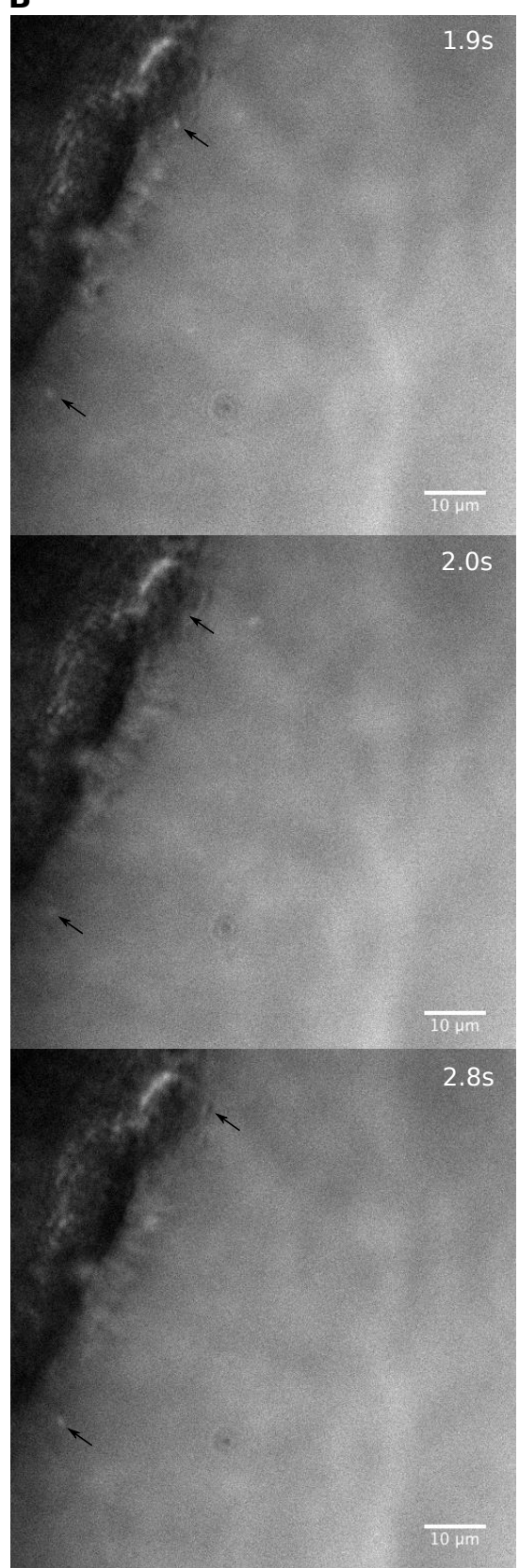

Figure 3.24: V3V explant was cut into thin strips and ciliary beating recorded from a lateral view. Fluorescently-labeled Z310 choroid plexus cells were added and transport along the cilia mediated flow observed. A Fluorescent EVs and aggregated were observed to be transported along the ciliary flow and made contact with ependymal cilia. B shows EVs in contact with ependymal cilia. The black arrows indicate the position of observed EVs. 


\section{Influence of Z310 cells EVs on v3V ependymal cilia beating direction}

To evaluate wherever EVs secreted by Z310 cells change the cilia beating direction of cilia bundles in the $\mathrm{v} 3 \mathrm{~V}$, the cilia-driven flow was monitored before and after a $30 \mathrm{~min}$ long incubation of $\mathrm{v} 3 \mathrm{~V}$ explants in EV-containing solution. The experiment was performed at RT. Analyzed flow maps are shown in Fig. $3.25 \mathbf{A}$ and $\mathbf{B}$. The v3V flow pattern did not show any changes regarding the cilia beating direction after the incubation. Even $22 \mathrm{~h}$ after the incubation the cilia-generated flow direction remaind the same $(n=2$, four explants from two animals). This result suggests, that under the experimental conditions the cilia beating direction is not influenced by the presence and contact with Z310 EVs.

\subsubsection{Interaction of Z310 EVs with mouse sperm flagella}

Mouse sperm cells were freshly isolated and incubated at $37^{\circ} \mathrm{C}$ until use (Tab. 5.2). Extracellular vesicles were fluorescently-labeled using specific antibodies (Fig. 3.23). The movement of sperm cells and the fluorescent EVs was observed and recorded simultaneously. This experiment was performed in collaboration with the lab of Dr. Cecilia Lo of the University of Pittsburgh.

EVs were hit by beating sperm cell flagella, but did not attach to them. Even after longer contact time with an immotile flagellum, EVs did not stick to it (Fig. 3.26). Although EVs were observed sticking to the heads of sperm cells, the sperm cells did not show an overall fluorescence labeling, which had been observed after incubation with fluorescently-labeled liposomes. Based on the very different cell origins and function of the used EVs and the sperm cells, an interaction between the EVs and the sperm cells flagella was not expected. This was indeed the case.

\subsubsection{Specificity of EV binding}

Once released into the cilia-generated flow of the $\mathrm{v} 3 \mathrm{~V}$, EVs are distributed to specific locations or kept away from the surface. Conclusively, the binding to cilia depends on approximation to the surface by cilia-generated flow but it might also involve local surface properties that mediate site-specific interaction with the ependymal cilia. The impact of both regulatory mechanisms was addressed in more detail. Cilia beating is not homogenous across the ventricular surface with cilia in some areas beating with a significantly changed angle compared to others [103]. To rule out whether local differences in cilia-generated 
flow dynamics affect the interaction between vesicles and cilia, ependyma of a genetically modified mouse with pathological changes in cilia beating was used. The underlying mutation is in the gene Cdk15 and is a model for the human disease Cdkl5-associated early infantile epileptic encephalopathy involving early onset intractable seizure, severe mental retardation and autistic-like behavior [104]. The ciliary stroke in these mice is modified in a way that cilia-generated flow at the surface is reversed and this way, all particles in a certain distance from the ependyma are guided directly towards the cilia (personal communication R. Faubel, C. Lo). As result of these changes in flow dynamics, more vesicles are brought in proximity of the cilia and if stochastics of physical interaction was important, much stronger staining would be observed with no local preference. Following incubation with fluorescently-labeled liposomes, cilia were stained in the identical areas of the ventricle (the region of flow 6 and flow 7) as in wildtype mice (Fig. 5.7). Suggesting that the specificity of vesicle binding is based on other means than site-specific transport.

It was observed that liposomes are likely to attach to damaged cells of the brain parenchyma, including ependymal cells. In general, intact cellular surfaces are covered with membrane-bound proteins and only upon damage, lipids are directly exposed to the extracellular environment. It might be that the binding to damaged cells and the cilia membrane is mediated by the same mechanism and this is the interaction between vesicular and cellular lipids. Since the exosomal membrane is covered with membrane-bound proteins, binding of exosomes to damaged cells would not be expected. NIH-3T3 and Z310 cells were used for a wounding assay and incubated in exosome-containing medium (Fig. 5.2). Small fluorescent spots were observed randomly distributed over the cell layer. For both cell types, no specific attachment of Z310 derived EVs to damaged cells was observed, whereas liposomes attached to damaged NIH-3T3 cells. These results suggest, that the binding to damaged cells is a characteristic of liposomes but not Z310 derived exosomes labeled with antibodies. This supports the hypothesis of a direct interaction between lipids of the vesicular and cell membranes. 

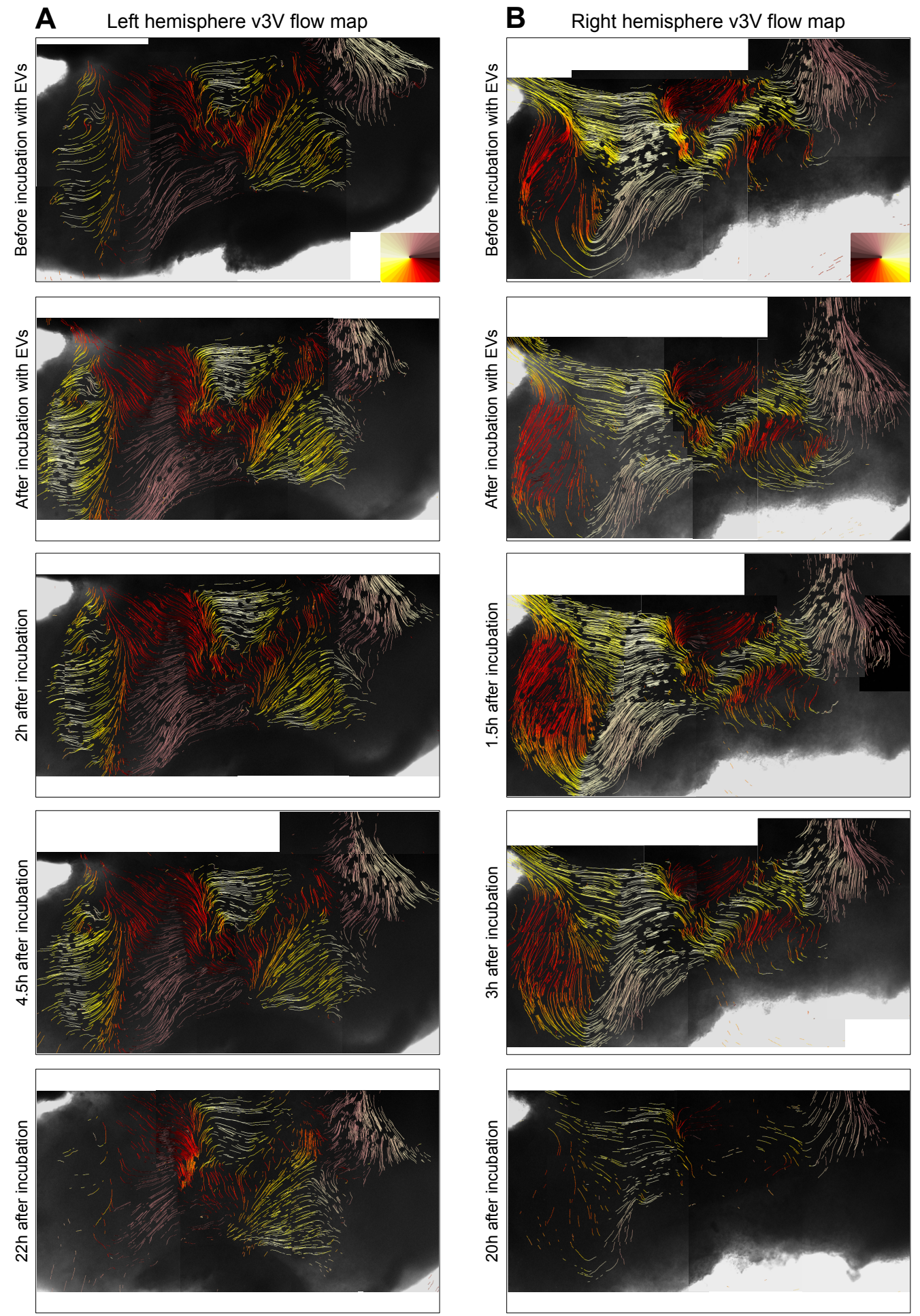

Figure 3.25: The cilia mediated flow of a v3V explant was recorded over time before and after incubation in homogeneous solution of Z310 cell EVs for $30 \mathrm{~min}$. Each recording was analyzed using particle tracking. The color code shown in the first row indicated the direction of movement (Sec. 2.4.2). The flow pattern does not change over time. 

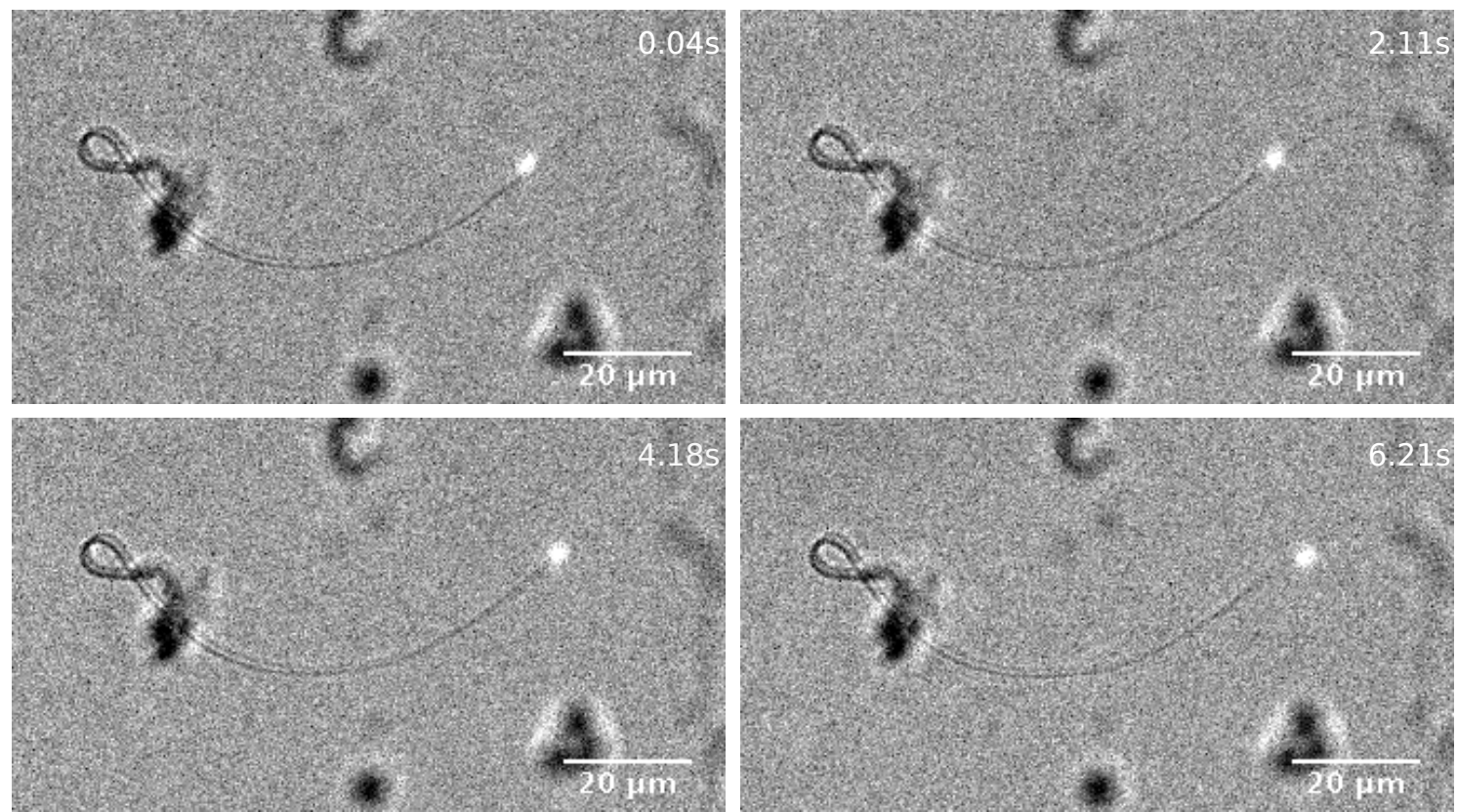

Figure 3.26: Mouse sperm cells were isolated and fluorescently-labeled Z310 choroid plexus cell EVs added to the solution. The image series shows the attachment and detachment of Z310 EVs to sperm flagellum. 
Chapter 3 Results 


\section{Chapter 4}

\section{Discussion}

Decades after the discovery of naturally occurring EVs, their initial view as cell debris has changed to a biologically relevant field of research that includes numerous applications in cancer research, medical application and intercellular communication $[6,42,53,55,56]$. Rising knowledge about the composition and content of EVs, source and target tissues and different mechanisms of production is the basis for identification of physiological processes that are regulated by EVs. A major aspect of EVs, specifically of exosomes, in CSF is the delivery to the target region. The circulation of CSF bulk flow is slow and hence inefficient as it promotes dilution of exosomes rather than directional transport. In contrast, transport via the ciliary network provides site-directed delivery across large distances. The results of this work reveal that the transport of lipid vesicles within the v3V is determined by the cilia-generated flow. Liposomes are transported with high precision and interact with ependymal cilia.

In addition, first steps have been taken to investigate the transport and interaction of choroid plexus derived extracellular vesicles within the v3V. Currently available protocols for isolation and labeling of exosomes are prone to generate artefacts. In this work, diverse methods were compared and optimized for isolation and labeling of Z310 derived exosomes. The identification of the most suitable method is the basis for studying the transport of choroid plexus derived exosomes by the cilia-generated flow network and identification of target sites in the brain. 


\subsection{Transport of extracellular vesicle like cargo in cilia-generated flow of the v3V}

Characterization of EV transport in the cilia-generated flow network of the $\mathrm{v} 3 \mathrm{~V}$ was performed with vesicles in their simplest form, the artificially produced liposomes. Protocols for production and labeling were established in the lab and optimized according to the experimental requirements. The transport properties were analysed and compared.

The application of small fluorescently-labeled liposome droplets into the cilia-generated near wall flow of the ventral third ventricle showed, that in most flow domains liposomes are transported closely along the ependymal wall in particular streamlines (Fig. 3.2, 3.3 and 3.5). Along these cilia prescribed streamlines, liposome crossed long distances in a few seconds. Apart from transport along single separated streamlines, liposome application also visualized splitting of streamlines into multiple single streams and the merging of several streamlines (Fig. 3.2 C, 3.3 C and F and 3.5 A and D). Multiple injections of liposome droplets on the same tissue explant and at the same location in the near wall flow led to transport of these liposomes along the exact same streamlines. In addition to that, liposomes transported along the streamlines also gave an approximal impression about the streamlines width. Based on this visualisation the width of streamlines was observed to: 1) widen slightly over the course of the streamline (Fig. 3.2 D, 3.3 C), 2) to widen and then to narrow again (Fig. 3.2 H, 3.3 D and E), or 3) to roughly stay the same (Fig. 3.2 G). Although the influence of diffusion cannot be excluded entirely, these results suggest, that the cilia-generated near wall flow dominates the transport of extracellular vesicle-like components of the CSF along the ventricular wall (also see App. 5.1). Therefore, the cilia-generated flow network allows precise delivery of extracellular vesicle-like cargo along the streamlines, connecting the site of vesicle release with a predetermined target site.

The transport of liposomes away from the ependymal wall of the $\mathrm{v} 3 \mathrm{~V}$ was observed in specific areas of the tissue explant e.g. at the end of flow 3 upon reaching the separatrix (Fig. 3.2 D). Thus, in addition to transport along the ventricular wall, transport across the ventricular lumen between the walls of the closed v3V may occur as well. Addressing the transport of CSF components in the closed $v 3 \mathrm{~V}$ experimentally is challenging. Even though magnetic resonance imaging was successfully used to analyse CSF bulk flow and CSF pulsation [7] the resolution does not allow a detailed study of cilia-generated near wall flow. To approach three-dimensional transport of CSF components within the closed 
ventricular system new techniques need to be developed.

\subsection{The interaction of extracellular vesicle-like CSF components with ependymal cells of the v3V}

The production and content of extracellular vesicles is known to depend on the source tissue and its physiological condition $[6,42]$. The results of this work showed that liposomes of specific lipid-compositions interact with multiciliated ependymal cells in defined regions of the v3V. Liposomes, whose membrane contained 1,2-dioleoyl-sn-glycero-3phospho-L-serine (DOPS) and cholesterol, were shown to attach to the motile cilia of ependymal cilia bundles in the region of flow 6 and flow 7 along the posterior duct (Fig. 3.7 and 5.5). However, not all cells of the ependymal wall in these flow regions show the same amount of liposome attachment. Even after exposure to a homogeneous solution of liposomes some cells of the ependymal wall seem to lack liposome attachment (Fig. 3.17 and 3.18). A possible explanation of this observation is the presence of other cell types within the ventricular wall of these regions. The region of flow 6 is located next to the $\mathrm{v} 3 \mathrm{~V}$ tanycyte region and contains a population of biciliated $\mathrm{E} 2$ cells embedded between the muticiliated ependymal cells [37]. A band of E2 cells was also located along the ventricular wall of the posterior duct [37]. These results suggest, that the observed liposome-cilia interaction is the result of a specific interaction between membrane components of the liposomal and motile ciliary membrane, of muticiliated cells within the v3V flow regions 6 and 7. Besides, a similar liposome-cilia interaction was not observed in any of the other ciliated tissues, like oviduct or human nasal epithelia cells, which were exposed to fluorescently-labeled liposomes (Sec. 3.2.6).

Both liposome membrane components, DOPS and cholesterol, are known to be involved in signalling processes $[65,73,99]$. However, it is not clear which predominantly causes the attachment to ependymal cilia. More experiments with liposomes of varying lipid-compositions will identify the required membrane components. This may help to investigate which component of the ciliary membrane contributes to the liposome-cilia attachment.

The ciliary membrane composition is known to be distinct from the rest of the cell membrane $[23,40]$. Nonetheless, liposome attachment to motile ependymal cilia of specific regions only, indicates a difference of the ciliary membrane composition throughout the ependymal cells of the $\mathrm{v} 3 \mathrm{~V}$ ventricular wall. These may present possible sites of 
extracellular vesicles interaction as well. Therefor, liposomes could be used to identify target sites of extracellular vesicles within the ventricular system of the brain. Further, lipid-composition depended interaction of liposomes with certain cells in the brain only, may proof useful for site specific delivery of vesicle encapsulate substances, as liposomes are also used as part of drug delivery systems $[64,76]$.

Since the major biological functions of lipids are diverse, it is tempting to speculate that the role of exosome attachment to cilia might exceed simple delivery of contained cargo. Transfer of energy is unlikely to be a function of exosome transfer as this is typically mediated by sugars that can be regulated and processed more efficiently. Assuming that exosomes integrate into the ciliary membrane similarly, the content would be taken up and the membrane would become a part of the ciliary membrane. As the ciliary membrane is not a simple extension of the cytoplasmic membrane and the composition is tightly regulated, transfer of membrane lipids from exosomes might be involved in regulation of cilium length. Recently, local variation of lipid compounds has been observed along the cilium that presumably forms compartmentalization of the membrane [40]. The uptake of exosomes would most probably modify the local composition. Together with membrane lipids, membrane proteins such as receptors would be transferred to the cilium. Various receptor proteins have been identified as components of exosomes, suggesting that, in addition to delivery of signalling compounds, exosomes transmit susceptibility of the target cell for reception of such. 


\section{Chapter 5}

\section{Appendix}

\subsection{Calculating the mean displacement of small particles caused by diffusion}

The directed movement of particles along a concentration gradient from a location of high concentration to a location of low concentration is called diffusion. The mean displacement distance $r_{D i f f}$ was calculated for small diffusing particles with a diameter $d$ of $30 \mathrm{~nm}$ and $80 \mathrm{~nm}$ in a volume (number of dimensions $n=3$ ) of an aqueous solution at a temperature $T=20^{\circ} \mathrm{C}$. The diffusion constant $D$ was calculated using the Stokes-Einstein equation [105]:

$$
D=\frac{k_{B} T}{6 \pi \eta R_{0}}
$$

with $k_{B}=$ Boltzmann constant, $\eta=$ dynamic viscosity, $R_{0}=$ hydrodynamic radius. The mean square displacement is given by:

$$
\left\langle r_{D i f f}^{2}(\tau)\right\rangle=2 n D \tau
$$

with $n=$ number of dimensions and $\tau=$ time. The displacement distance $r_{F l o w}$ caused by the cilia-generated flow was calculated with the equation:

$$
r_{\text {Flow }}=\bar{v} \tau
$$

$\bar{v}$ is the mean transport velocity of liposome droplets, which were applied to the ciliagenerated near wall flow and recorded using fluorescence microscopy (Sec. 3.1.3) at 


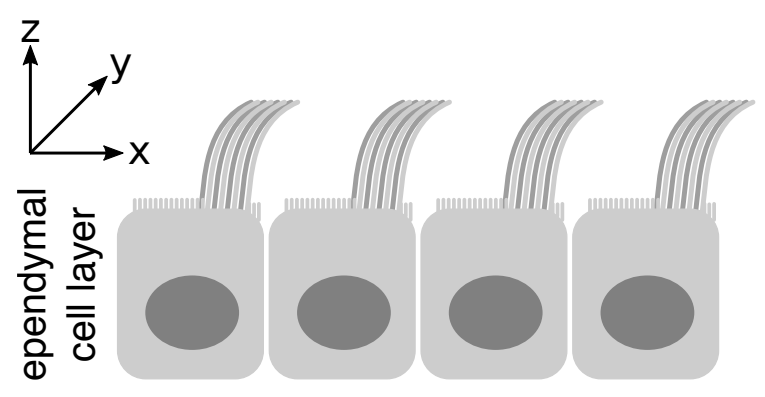

Figure 5.1: Schema of coordinates in regard to the ependymal wall.

room temperature $\left(T=20\right.$ to $\left.21^{\circ} \mathrm{C}\right)$. Therefore, $\bar{v}$ was calculated from the movement of the applied droplets in $\mathrm{x}$ and $\mathrm{y}$ direction (dimension $n=2$ ) parallel to the ependymal wall and does not include transport in $\mathrm{z}$ direction (Fig. 5.1). The results for $\tau=3 \mathrm{~s}$ are shown in Tab. 5.1. The displacement of $30 \mathrm{~nm}$ to $80 \mathrm{~nm}$ large particles caused by diffusion is 35 to 57 times smaller than the transport by cilia-generated flow.

\begin{tabular}{c||c||c}
$\mathrm{d}[\mathrm{nm}]$ & $\begin{array}{c}\text { diffusion } \\
r_{\text {Diff }}[\mu \mathrm{m}]\end{array}$ & $\begin{array}{c}\text { cilia-generated flow } \\
r_{\text {Flow }}[\mu \mathrm{m}]\end{array}$ \\
\hline \hline 30 & 16.1 & 561 \\
80 & 9.8 & 561
\end{tabular}

Table 5.1: The mean displacement distance of $30 \mathrm{~nm}$ and $80 \mathrm{~nm}$ large particles caused diffusion $r_{\text {Diff }}$ and cilia-generated flow $r_{\text {Flow }}$ was calculated. Parameters for diffusion: $T=20^{\circ} \mathrm{C}, n=3$, $\eta=1 \mathrm{mPas}, \tau=3 \mathrm{~s}$. Parameters for cilia-generated flow: $T=20$ to $21^{\circ} \mathrm{C}, n=2, \bar{v}=187 \mu \mathrm{m} / \mathrm{s}$, $\tau=3 \mathrm{~s}$. 


\subsection{List of experiments and mice}

\begin{tabular}{|c|c|c|c|c|c|}
\hline Experiment & Figures & $\begin{array}{l}\text { age } \\
\text { (weeks) }\end{array}$ & gender & genotype/ tissue* & background \\
\hline $\begin{array}{l}\text { Propagation of liposomes } \\
\text { in ciliary near wall flow }\end{array}$ & $3.2,3.3,3.5$ & 10 to 12 & male & WT $(n=12)$ & C57BL/6N \\
\hline $\begin{array}{l}\text { Velocity distribution of li- } \\
\text { posomes in cilia near wall } \\
\text { flow }\end{array}$ & 3.4 & 10 to 12 & male & WT $(n=7)$ & C57BL/6N \\
\hline $\begin{array}{l}\text { Localization of liposome } \\
\text { attachment in } \mathrm{v} 3 \mathrm{~V}\end{array}$ & 3.7 & 10 to 13 & male & WT $(n=9)$ & C57BL/6N \\
\hline $\begin{array}{l}\text { Flow map and velocity of } \\
\mathrm{v} 3 \mathrm{~V} \text { before and after lipo- } \\
\text { some incubation }\end{array}$ & $3.9,3.9$ & 9 to 20 & male & $\begin{array}{l}\text { WT } \\
\text { (shown one, observed for } n=6 \text {, } \\
\text { negativ control } n=2 \text { ) }\end{array}$ & C57BL/6N \\
\hline $\begin{array}{l}\text { Cilia beating frequency } \\
\text { before and after adding li- } \\
\text { posomes }\end{array}$ & 3.11 & 8 & male & WT $(n=1)$ & $\mathrm{C} 57 \mathrm{BL} / 6 \mathrm{~J}$ \\
\hline $\begin{array}{l}\text { Confocal live imaging of } \\
\text { liposome attachment to } \\
\mathrm{v} 3 \mathrm{~V} \text { cilia }\end{array}$ & $3.12,3.13$ & 6 & male & WT $(n=1)$ & C57BL/6J \\
\hline $\begin{array}{l}\text { Liposome lipid composi- } \\
\text { tion test }\end{array}$ & $\begin{array}{l}3.14, \quad 3.15, \quad 5.3 \\
5.4,5.5,5.6\end{array}$ & 9 to 24 & male & WT $(n=19)$ & C57BL/6N \\
\hline $\begin{array}{l}\text { Transport of liposomes } \\
\text { along v3V side view } \\
\text { preparation }\end{array}$ & 3.16 & $\begin{array}{l}14 \\
14 \\
14\end{array}$ & $\begin{array}{l}\text { female } \\
\text { female } \\
\text { male }\end{array}$ & $\begin{array}{l}\text { Sap130 +/f, Foxj1 +/cre }) \\
(\text { Sap130 +/f }) \\
(\operatorname{Lrp} 2+/ f)\end{array}$ & $\begin{array}{l}\mathrm{C} 57 \mathrm{BL} / 6 \mathrm{~J} \\
\mathrm{C} 57 \mathrm{BL} / 6 \mathrm{~J} \\
\mathrm{C} 57 \mathrm{BL} / 6 \mathrm{~J}\end{array}$ \\
\hline $\begin{array}{l}\text { Localisation of liposome } \\
\text { attachment }\end{array}$ & $3.17,3.18$ & 10 to 18 & male & $\begin{array}{l}\text { WT }(n=5) \\
\text { negativ control }(n=2)\end{array}$ & $\mathrm{C} 57 \mathrm{BL} / 6 \mathrm{~N}$ \\
\hline $\begin{array}{l}\text { Kinetic of liposome at- } \\
\text { tachment to v3V cilia }\end{array}$ & $3.19,3.20,3.21$ & 9 to 20 & male & WT $(n=9)$ & C57BL/6N \\
\hline $\begin{array}{l}\text { Attachment of liposomes } \\
\text { to cilia and flagella }\end{array}$ & 3.22 & $\begin{array}{l}12 \\
12 \\
11 \\
8\end{array}$ & $\begin{array}{l}\text { male } \\
\text { male } \\
\text { male } \\
\text { female }\end{array}$ & $\begin{array}{l}\text { WT (sperm) } \\
\text { WT (sperm) } \\
\text { (Lrp2 +/f) (sperm) } \\
\text { WT (fallopian tube) }\end{array}$ & $\begin{array}{l}\text { C57BL/6J } \\
\text { C57BL/6J } \\
\text { C57BL/6J } \\
\text { CD1 }\end{array}$ \\
\hline $\begin{array}{l}\text { Transport of } \mathrm{Z310} \text { cell } \\
\text { EVs along v3V side view } \\
\text { preparation }\end{array}$ & 3.24 & $\begin{array}{l}8 \\
8 \\
8 \\
8 \\
8 \\
\end{array}$ & $\begin{array}{l}\text { male } \\
\text { male } \\
\text { male } \\
\text { female } \\
\text { male }\end{array}$ & $\begin{array}{l}\text { Sap130 +/f) } \\
(\text { Sap130 +/f) } \\
(\text { Sap130 +/f) } \\
(\text { Dnahc5 +/m) } \\
(\text { Sap130 +/f) }\end{array}$ & $\begin{array}{l}\mathrm{C} 57 \mathrm{BL} / 6 \mathrm{~J} \\
\mathrm{C} 57 \mathrm{BL} / 6 \mathrm{~J} \\
\mathrm{C} 57 \mathrm{BL} / 6 \mathrm{~J} \\
\mathrm{C} 57 \mathrm{BL} / 6 \mathrm{~J} \\
\mathrm{C} 57 \mathrm{BL} / 6 \mathrm{~J} \\
\end{array}$ \\
\hline $\begin{array}{l}\text { Flow maps of v3V before } \\
\text { and after incubation with } \\
\text { Z310 cell EVs }\end{array}$ & 3.25 & 18 & male & WT $(n=2)$ & C57BL/6N \\
\hline $\begin{array}{l}\text { Interaction of Z310 cell } \\
\text { EVs with sperm flagella }\end{array}$ & 3.26 & 8 & male & $($ Sap130 +/f) $($ sperm $)$ & $\mathrm{C} 57 \mathrm{BL} / 6 \mathrm{~J}$ \\
\hline $\begin{array}{l}\text { Liposome interaction with } \\
\text { ependymal cilia of Cdk15 } \\
\text { mutant }\end{array}$ & 5.7 & $\begin{array}{l}6 \\
6\end{array}$ & $\begin{array}{l}\text { male } \\
\text { male }\end{array}$ & $\begin{array}{l}(\mathrm{Cdk15}-/-) \\
(\mathrm{Cdk15}-/-)\end{array}$ & $\begin{array}{l}\mathrm{C} 57 \mathrm{BL} / 6 \mathrm{~J} \\
\mathrm{C} 57 \mathrm{BL} / 6 \mathrm{~J}\end{array}$ \\
\hline
\end{tabular}

Table 5.2: List of experiments with corresponding figures and used mice. * If not noted otherwise ependymal tissue was used. The $\mathrm{n}$ represents the number of used animals. If $\mathrm{n}$ is not listed only the animals listed were used. WT $=$ wild type, $\mathrm{f}=$ floxed allele,$+=$ wild type allele,$-=$ knockout, $\mathrm{m}=$ mutant allele. 


\subsection{Figures}

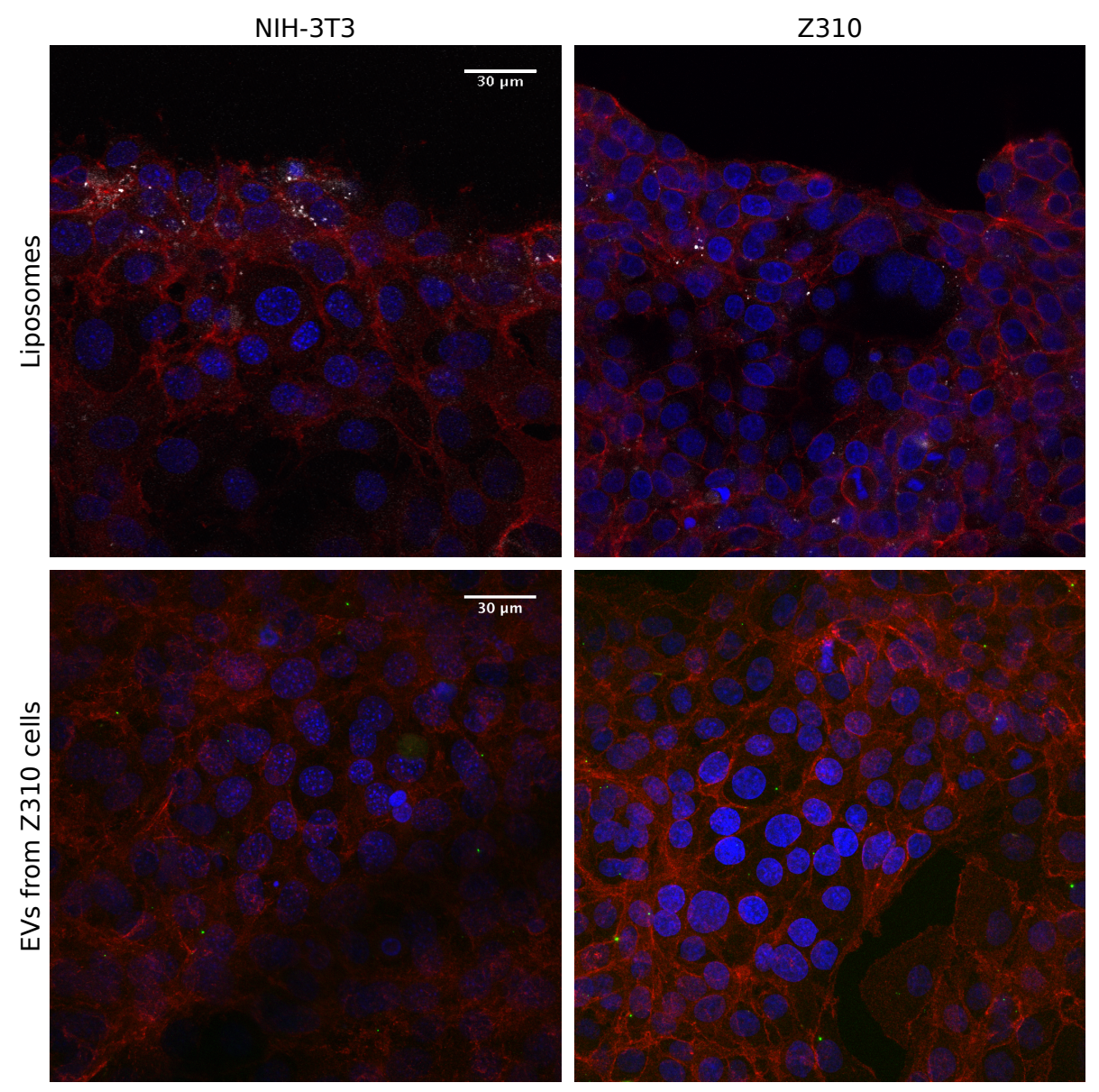

Figure 5.2: Layers of NIH-3T3 fibroblasts and Z310 rat choroid plexus cells were injured using a piped tip. Then, the cells were incubated with fluorescently-labeled liposomes of composition II (white, Tab. 2.1, top row) or fluorescently-labeled EVs isolated from Z310 cell supernatant (green, bottom row). Afterwards, the cells were fixed and stained with DAPI (blue) and $\beta$ catenin (red). Damaged NIH-3T3 cells show a strong attachment of liposomes, while Z310 cells show less and unspecific attachment over the whole cell assay. Damaged NIH-3T3 and Z310 cells incubated in EVs both show unspecific attachment of EVs over the entire field. 


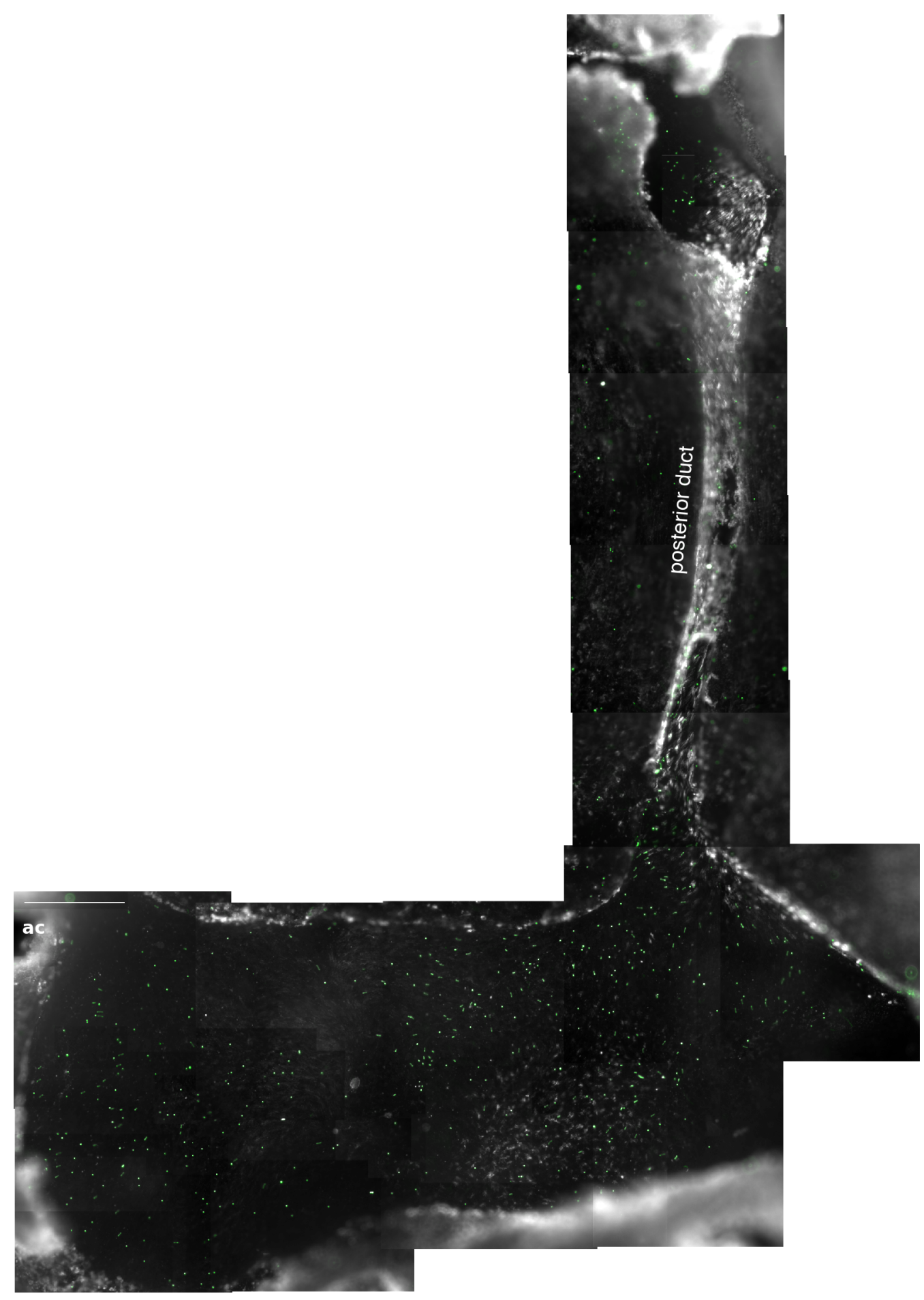

Figure 5.3: A v3V explant was incubated in fluorescently-labeled liposome solution in a dilution of 1:250 for $1 \mathrm{~h}$. Liposomes were of composition III (Tab. 2.1), prepared by size exclusion chromatography. The fluorescent signal of liposomes shown in white, fluorescent beads shown in green. Liposomes attached strongly in the region the of flow 6 and in flow 7 along the posterior duct. Cells of the surrounding brain parenchyma show strong liposome attachment. Scale bar $=300 \mu \mathrm{m}$, ac $=$ anterior commissure. 


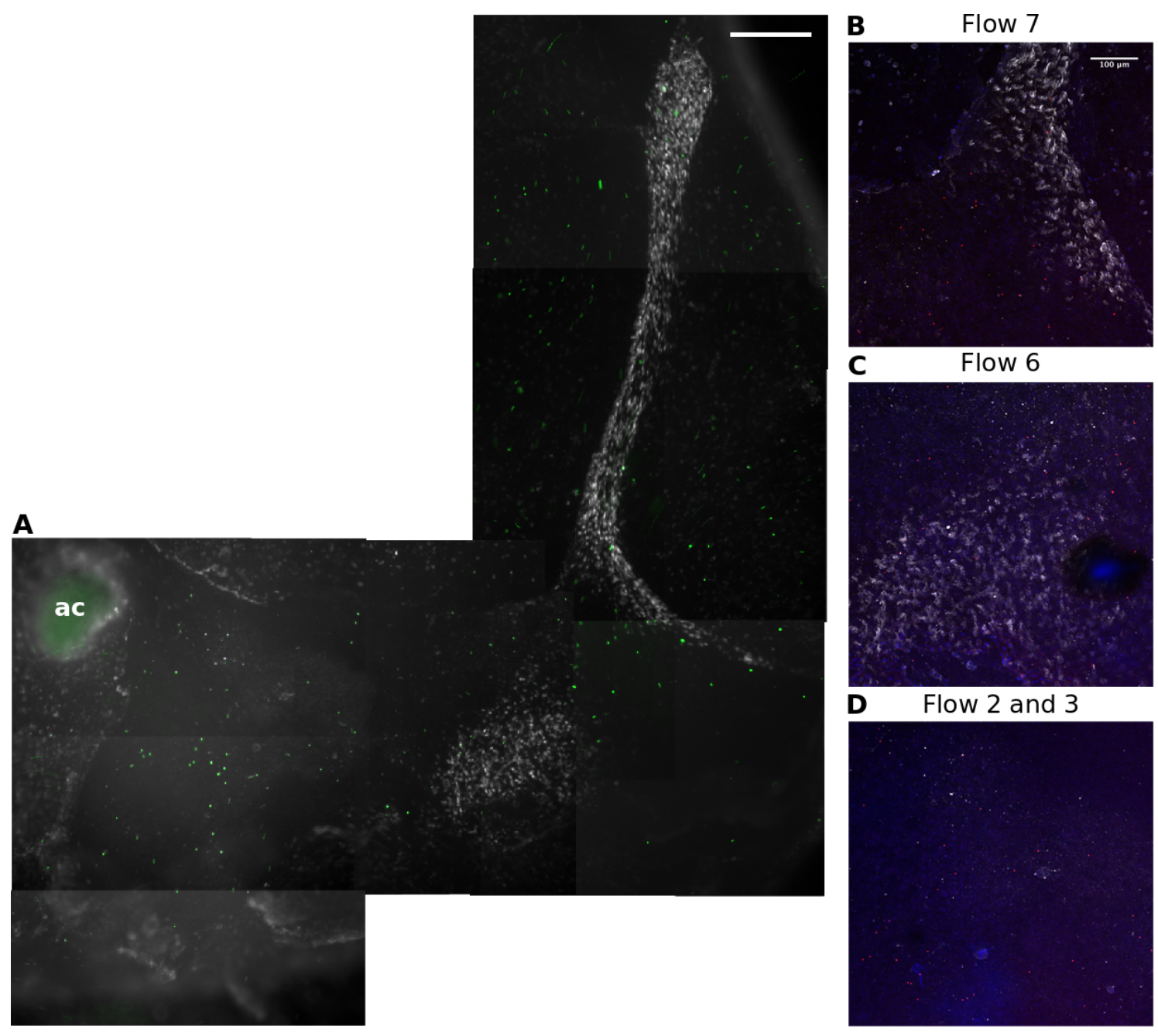

Figure 5.4: V3V explant was incubated in highly diluted fluorescently-labeled liposome solution of composition IV (Tab. 2.1) for $10 \mathrm{~min}$. A v3V explant after incubation in liposome (white) solution. Fluorescent beads stuck on the ependymal surface during ciliary flow recording are shown in green. Scale $b a r=300 \mu \mathrm{m}, \mathrm{ac}=$ anterior commissure. Liposomes are attached strongest in the region of flow 6 and flow 7 along the posterior duct to the dorsal $3 \mathrm{~V}$. Cells in the surrounding brain parenchyma show liposome attachment. B, C and D show projected confocal stacks after fixation and staining of v3V explant after incubation with fluorescently-labeled liposomes (white). Tissue was stained with DAPI (blue), fluorescent beads are shown in red. 


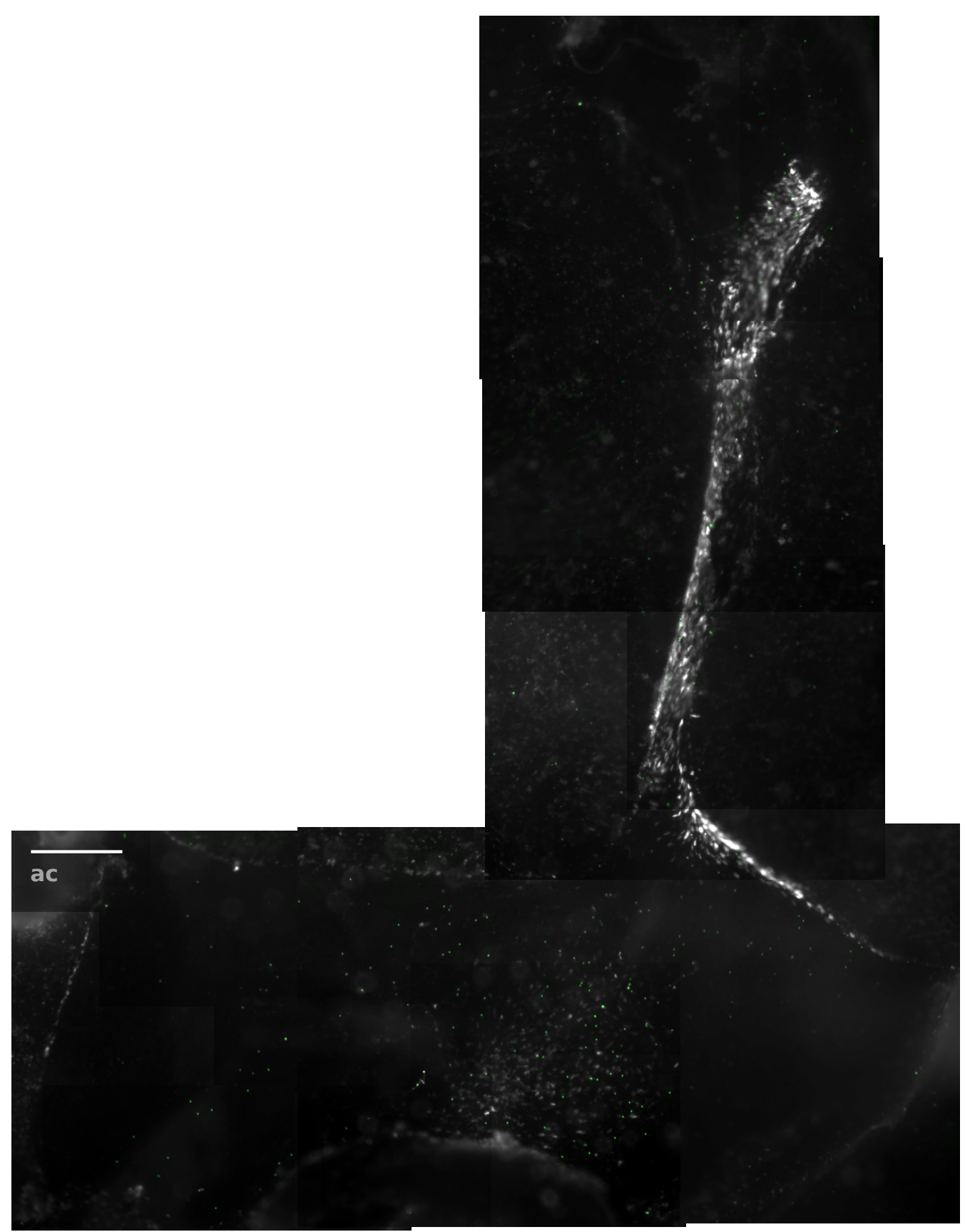

Figure 5.5: A v3V explant was incubated in fluorescently-labeled liposome solution in a dilution of 1:500 for $10 \mathrm{~min}$. Liposomes were prepared by extrusion of composition VIII (Tab. 2.2). Fluorescent signal of liposomes is shown in white, fluorescent beads are shown in green. The strongest attachment of liposomes is visible in the region of flow 6 and flow 7 along the posterior duct up to the dorsal $3 \mathrm{~V}$. Cells of the surrounding brain parenchyma show liposome attachment too. Scale bar $=300 \mu \mathrm{m}, \mathrm{ac}=$ anterior commissure. 


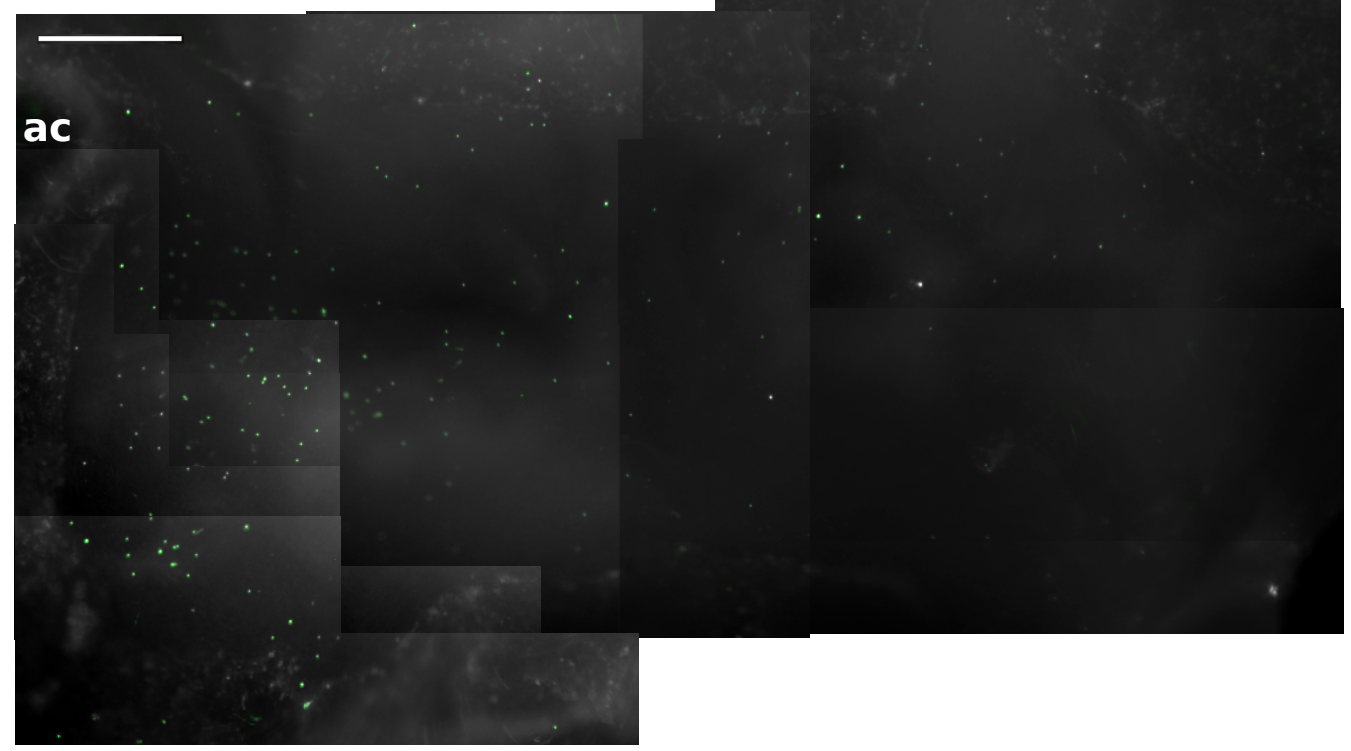

Figure 5.6: A v3V explant was incubated in fluorescently-labeled liposome solution in a dilution of 1:500 for $10 \mathrm{~min}$. Liposomes were prepared by extrusion of composition XI (Tab. 2.2). Fluorescent signal of liposomes is shown in white, fluorescent beads are shown in green. The region of flow 6 and flow 7 show no attachment of liposomes, while cells of the brain parenchyma still show liposome attachment. Scale bar $=300 \mu \mathrm{m}, \mathrm{ac}=$ anterior commissure.

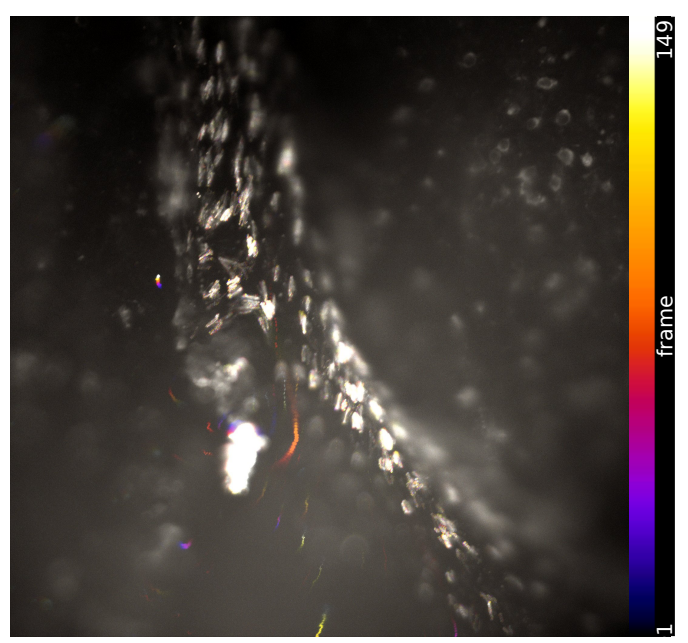

Figure 5.7: v3V explant of Cdk15 mutant mouse was incubated in fluorescently-labeled liposome solution of composition II (Tab. 2.1) prepared by size exclusion chromatography. Cilia bundles of the mutant showed high liposome attachment in the same region (flow 7) as observed in the wildtype (compare to Fig. 3.7). 


\section{Bibliography}

[1] Del Bigio, M. R. Ependymal cells: biology and pathology. Acta Neuropathologica, 119(1):55-73, 2010. Cited on p. v, 2, 3, 5, 44

[2] Faubel, R., Westendorf, C., Bodenschatz, E., and Eichele, G. Cilia-based flow network in the brain ventricles. Science, 353(6295):176-8, Jul 2016. Cited on p. v, $1,3,4,23,40,45,48$

[3] Balusu, S., Van Wonterghem, E., De Rycke, R., Raemdonck, K., Stremersch, S., Gevaert, K., Brkic, M., Demeestere, D., Vanhooren, V., Hendrix, A., Libert, C., and Vandenbroucke, R. E. Identification of a novel mechanism of blood-brain communication during peripheral inflammation via choroid plexus-derived extracellular vesicles. EMBO Molecular Medicine, 8(10):1162-1183, 10 2016. Cited on p. v, 1, 9,80

[4] Abels, E. R. and Breakefield, X. O. Introduction to extracellular vesicles: Biogenesis, rna cargo selection, content, release, and uptake. Cellular and molecular neurobiology, 36(3):301-312, 04 2016. Cited on p. v, 37

[5] Grapp, M., Wrede, A., Schweizer, M., Huewel, S., Galla, H.-J., Snaidero, N., Simons, M., Bückers, J., Low, P. S., Urlaub, H., and et al. Choroid plexus transcytosis and exosome shuttling deliver folate into brain parenchyma. Nature Communications, 4(1), Jul 2013. ISSN 2041-1723. Cited on p. v, 1, 8, 80

[6] Colombo, M., Raposo, G., and Théry, C. Biogenesis, secretion, and intercellular interactions of exosomes and other extracellular vesicles. Annual Review of Cell and Developmental Biology, 30(1):255-289, 2018/07/25 2014. Cited on p. v, 6, 7, $8,9,89,91$

[7] Hladky, S. B. and Barrand, M. A. Mechanisms of fluid movement into, through 
and out of the brain: evaluation of the evidence. Fluids and Barriers of the CNS, 11(1):26, 2014. ISSN 2045-8118. Cited on p. 1, 2, 90

[8] Haraszti, R. A., Didiot, M.-C., Sapp, E., Leszyk, J., Shaffer, S. A., Rockwell, H. E., Gao, F., Narain, N. R., DiFiglia, M., Kiebish, M. A., and et al. High-resolution proteomic and lipidomic analysis of exosomes and microvesicles from different cell sources. Journal of Extracellular Vesicles, 5(1):32570, Jan 2016. ISSN 20013078. Cited on p. 1, 80

[9] Lasic, D. D. The mechanism of vesicle formation. Biochemical Journal, 256(1): 1-11, 11 1988. Cited on p. 1, 11

[10] Akbarzadeh, A., Rezaei-Sadabady, R., Davaran, S., Joo, S. W., Zarghami, N., Hanifehpour, Y., Samiei, M., Kouhi, M., and Nejati-Koshki, K. Liposome: classification, preparation, and applications. Nanoscale Research Letters, 8(1):102-102, 2013. Cited on p. 1, 10, 11, 15, 60, 67

[11] Dale, P., George J, A., David, F., Lawrence C, K., Anthony-Samuel, L., James O, M., and S Mark, W., editors. Neuroscience. Sinauer Associates Inc, 2 edition, 2001. Cited on p. 2

[12] Damkier, H. H., Brown, P. D., and Praetorius, J. Cerebrospinal fluid secretion by the choroid plexus. Physiological Reviews, 93(4):1847-1892, 2018/11/22 2013. Cited on p. 2

[13] Worthington, W. C. and Cathcart, R. S. Ependymal cilia: Distribution and activity in the adult human brain. Science, 139(3551):221-222, 1963. ISSN 0036-8075. Cited on p. 2, 6

[14] Mirzadeh, Z., Han, Y.-G., Soriano-Navarro, M., García-Verdugo, J. M., and Alvarez-Buylla, A. Cilia organize ependymal planar polarity. The Journal of neuroscience : the official journal of the Society for Neuroscience, 30(7):2600-2610, 02 2010. Cited on p. 2

[15] Sawamoto, K., Wichterle, H., Gonzalez-Perez, O., Cholfin, J. A., Yamada, M., Spassky, N., Murcia, N. S., Garcia-Verdugo, J. M., Marin, O., Rubenstein, J. L. R., Tessier-Lavigne, M., Okano, H., and Alvarez-Buylla, A. New neurons follow the 
flow of cerebrospinal fluid in the adult brain. Science, 311(5761):629-632, 2006. ISSN 0036-8075. Cited on p. 2, 6

[16] Fischer, R. S. Move your microvilli. The Journal of Cell Biology, 207(1):9-11, 10 2014. Cited on p. 2

[17] Liddelow, S. A. Development of the choroid plexus and blood-csf barrier. Frontiers in neuroscience, 9:32; 32-32, 03 2015. Cited on p. 2

[18] Dreha-Kulaczewski, S., Joseph, A. A., Merboldt, K.-D., Ludwig, H.-C., Gärtner, J., and Frahm, J. Inspiration is the major regulator of human csf flow. The Journal of Neuroscience, 35(6):2485, 02 2015. Cited on p. 2

[19] Siyahhan, B., Knobloch, V., de Zélicourt, D., Asgari, M., Schmid Daners, M., Poulikakos, D., and Kurtcuoglu, V. Flow induced by ependymal cilia dominates near-wall cerebrospinal fluid dynamics in the lateral ventricles. Journal of the Royal Society, Interface, 11(94):20131189; 20131189-20131189, 2014. Cited on p. 3

[20] Stadlbauer, A., Salomonowitz, E., van der Riet, W., Buchfelder, M., and Ganslandt, O. Insight into the patterns of cerebrospinal fluid flow in the human ventricular system using mr velocity mapping. NeuroImage, 51(1):42-52, 2010. Cited on p.

[21] Daouk, J., Bouzerar, R., and Baledent, O. Heart rate and respiration influence on macroscopic blood and csf flows. Acta Radiologica, 58(8):977-982, 2019/04/20 2016. Cited on p. 2

[22] Fliegauf, M., Benzing, T., and Omran, H. When cilia go bad: cilia defects and ciliopathies. Nature Reviews Molecular Cell Biology, 8:880 EP -, 11 2007. Cited on p. 4

[23] Khan, S. and Scholey, J. M. Assembly, functions and evolution of archaella, flagella and cilia. Current Biology, 28(6):R278-R292, 2018/07/25 2018. Cited on p. 4, $5,6,91$

[24] Bolborea, M. and Dale, N. Hypothalamic tanycytes: potential roles in the control of feeding and energy balance. Trends in Neurosciences, 36(2):91-100, Feb 2013. ISSN 0166-2236. Cited on p. 3 
[25] Spassky, N., Merkle, F. T., Flames, N., Tramontin, A. D., García-Verdugo, J., and Alvarez-Buylla, A. Adult ependymal cells are postmitotic and are derived from radial glial cells during embryogenesis. The Journal of Neuroscience, 25(1):10, 01 2005. Cited on p. 3

[26] Redzic, Z. B., Preston, J. E., Duncan, J. A., Chodobski, A., and SzmydyngerChodobska, J. The Choroid Plexus-Cerebrospinal Fluid System: From Development to Aging, volume 71, pages 1-52. Academic Press, 2005. ISBN 0070-2153. Cited on p. 3

[27] Mirzadeh, Z., Merkle, F. T., Soriano-Navarro, M., Garcia-Verdugo, J. M., and Alvarez-Buylla, A. Neural stem cells confer unique pinwheel architecture to the ventricular surface in neurogenic regions of the adult brain. Cell stem cell, 3(3): 265-278, 09 2008. Cited on p. 5

[28] Goodman, T. and Hajihosseini, M. K. Hypothalamic tanycytes-masters and servants of metabolic, neuroendocrine, and neurogenic functions. Frontiers in Neuroscience, 9, Oct 2015. ISSN 1662-453X. Cited on p. 5

[29] Ebling, F. J. P. and Lewis, J. E. Tanycytes and hypothalamic control of energy metabolism. Glia, 66(6):1176-1184, Feb 2018. ISSN 0894-1491. Cited on p. 5

[30] Satir, P., Heuser, T., and Sale, W. S. A structural basis for how motile cilia beat. Bioscience, 64(12):1073-1083, 12 2014. Cited on p. 5

[31] Malicki, J. J. and Johnson, C. A. The cilium: Cellular antenna and central processing unit. Trends in Cell Biology, 27(2):126-140, 2018/07/29 2017. Cited on p. 48

[32] Drummond, I. A. Cilia functions in development. Current opinion in cell biology, 24(1):24-30, 02 2012. Cited on $p$.

[33] Oh, E. C. and Katsanis, N. Cilia in vertebrate development and disease. Development (Cambridge, England), 139(3):443-448, 02 2012. Cited on p.

[34] Wood, C. R., Huang, K., Diener, D. R., and Rosenbaum, J. L. The cilium secretes bioactive ectosomes. Current Biology, 23(10):906-911, 2018/07/29 2013. Cited on $\mathrm{p}$. 
[35] Nager, A. R., Goldstein, J. S., Herranz-Pérez, V., Portran, D., Ye, F., GarciaVerdugo, J. M., and Nachury, M. V. An actin network dispatches ciliary gpcrs into extracellular vesicles to modulate signaling. Cell, 168(1):252-263.e14, 2018/07/29 2017. Cited on p. 5, 6

[36] Phua, S. C., Chiba, S., Suzuki, M., Su, E., Roberson, E. C., Pusapati, G. V., Setou, M., Rohatgi, R., Reiter, J. F., Ikegami, K., and Inoue, T. Dynamic remodeling of membrane composition drives cell cycle through primary cilia excision. Cell, 168 (1):264-279.e15, 2018/07/29 2017. Cited on p. 6

[37] Mirzadeh, Z., Kusne, Y., Duran-Moreno, M., Cabrales, E., Gil-Perotin, S., Ortiz, C., Chen, B., Garcia-Verdugo, J. M., Sanai, N., and Alvarez-Buylla, A. Bi- and uniciliated ependymal cells define continuous floor-plate-derived tanycytic territories. Nature communications, 8:13759; 13759-13759, 01 2017. Cited on p. 6, 91

[38] Ohata, S. and Alvarez-Buylla, A. Planar organization of multiciliated ependymal (e1) cells in the brain ventricular epithelium. Trends in neurosciences, 39(8):543551, 08 2016. Cited on $\mathrm{p}$.

[39] O’Callaghan, C. L., Sikand, K., Rutman, A., and Hirst, R. A. The effect of viscous loading on brain ependymal cilia. Neuroscience Letters, 439(1):56-60, 2008. Cited on p. 6

[40] Garcia, I., Galo, Raleigh, D. R., and Reiter, J. F. How the ciliary membrane is organized inside-out to communicate outside-in. Current Biology, 28(8):R421R434, 2018/07/04 2018. Cited on p. 6, 60, 77, 91, 92

[41] Raposo, G. and Stoorvogel, W. Extracellular vesicles: Exosomes, microvesicles, and friends. The Journal of Cell Biology, 200(4):373, 02 2013. Cited on p. 6, 7, 8

[42] Cocucci, E. and Meldolesi, J. Ectosomes and exosomes: shedding the confusion between extracellular vesicles. Trends in Cell Biology, 25(6):364-372, 2018/07/25 2015. Cited on p. 6, 7, 8, 9, 89, 91

[43] Henne, W. M., Buchkovich, N. J., and Emr, S. D. The escrt pathway. Developmental Cell, 21(1):77-91, 2018/07/25 2011. Cited on p. 7 
[44] Bissig, C. and Gruenberg, J. Alix and the multivesicular endosome: Alix in wonderland. Trends in Cell Biology, 24(1):19-25, 2018/07/25 2014. Cited on p. 7

[45] Hanson, P. I. and Cashikar, A. Multivesicular body morphogenesis. Annual Review of Cell and Developmental Biology, 28(1):337-362, 2018/07/25 2012. Cited on p. 7

[46] Trajkovic, K., Hsu, C., Chiantia, S., Rajendran, L., Wenzel, D., Wieland, F., Schwille, P., Brügger, B., and Simons, M. Ceramide triggers budding of exosome vesicles into multivesicular endosomes. Science, 319(5867):1244, 02 2008. Cited on p. 8

[47] Caby, M.-P., Lankar, D., Vincendeau-Scherrer, C., Raposo, G., and Bonnerot, C. Exosomal-like vesicles are present in human blood plasma. International Immunology, 17(7):879-887, 07 2005. Cited on p. 8

[48] Ogawa, Y., Miura, Y., Harazono, A., Kanai-Azuma, M., Akimoto, Y., Kawakami, H., Yamaguchi, T., Toda, T., Endo, T., Tsubuki, M., and Yanoshita, R. Proteomic analysis of two types of exosomes in human whole saliva. Biological and Pharmaceutical Bulletin, 34(1):13-23, 2011. Cited on p. 8

[49] Ronquist, G. and Brody, I. The prostasome: its secretion and function in man. Biochimica et Biophysica Acta (BBA) - Reviews on Biomembranes, 822(2):203218, 1985. Cited on p. 8

[50] Asea, A., Jean-Pierre, C., Kaur, P., Rao, P., Linhares, I. M., Skupski, D., and Witkin, S. S. Heat shock protein-containing exosomes in mid-trimester amniotic fluids. Journal of Reproductive Immunology, 79(1):12-17, 2008. Cited on p. 8

[51] Pisitkun, T., Shen, R.-F., and Knepper, M. A. Identification and proteomic profiling of exosomes in human urine. Proceedings of the National Academy of Sciences of the United States of America, 101(36):13368, 09 2004. Cited on p. 8

[52] LJ, V., RA, S., VA, L., CL, M., R, C., and AF, H. Packaging of prions into exosomes is associated with a novel pathway of prp processing. The Journal of Pathology, 211(5):582-590, 2018/07/25 2007. Cited on p. 8 
[53] Valadi, H., Ekström, K., Bossios, A., Sjöstrand, M., Lee, J. J., and Lötvall, J. O. Exosome-mediated transfer of mrnas and micrornas is a novel mechanism of genetic exchange between cells. Nature Cell Biology, 9:654 EP -, 05 2007. Cited on p. 9,89

[54] Chevallier, J., Chamoun, Z., Jiang, G., Prestwich, G., Sakai, N., Matile, S., Parton, R. G., and Gruenberg, J. Lysobisphosphatidic acid controls endosomal cholesterol levels. Journal of Biological Chemistry, 283(41):27871-27880, 10 2008. Cited on p. 9

[55] Théry, C. Exosomes: secreted vesicles and intercellular communications. F1000 Biology Reports, 3:15, 2011. Cited on p. 9, 89

[56] Stahl, P. D. and Raposo, G. Exosomes and extracellular vesicles: the path forward. Essays In Biochemistry, 62(2):119, 05 2018. Cited on p. 9, 89

[57] Xu, B., Zhang, Y., Du, X.-F., Li, J., Zi, H.-X., Bu, J.-W., Yan, Y., Han, H., and Du, J.-L. Neurons secrete mir-132-containing exosomes to regulate brain vascular integrity. Cell Research, 27:882 EP -, 04 2017. Cited on p. 9

[58] Segura, E., Guérin, C., Hogg, N., Amigorena, S., and Théry, C. $\mathrm{Cd} 8<\sup >+</$ sup $>$ dendritic cells use lfa- 1 to capture mhc-peptide complexes from exosomes in vivo. The Journal of Immunology, 179(3):1489, 08 2007. Cited on p. 9

[59] Morelli, A. E., Larregina, A. T., Shufesky, W. J., Sullivan, M. L. G., Stolz, D. B., Papworth, G. D., Zahorchak, A. F., Logar, A. J., Wang, Z., Watkins, S. C., Falo, L. D., and Thomson, A. W. Endocytosis, intracellular sorting, and processing of exosomes by dendritic cells. Blood, 104(10):3257, 11 2004. Cited on p. 9

[60] Tian, T., Zhu, Y.-L., Zhou, Y.-Y., Liang, G.-F., Wang, Y.-Y., Hu, F.-H., and Xiao, Z.-D. Exosome uptake through clathrin-mediated endocytosis and macropinocytosis and mediating mir-21 delivery. The Journal of Biological Chemistry, 289(32): 22258-22267, 08 2014. Cited on p. 9

[61] van Meer, G., Voelker, D. R., and Feigenson, G. W. Membrane lipids: where they are and how they behave. Nature Reviews Molecular Cell Biology, 9:112 EP -, 02 2008. Cited on p. 10 
[62] Buehler, L. K. Cell Membranes. Garland Science, Taylor and Francis Group, 2015. Cited on p. 10

[63] Alberts, B., Johnson, A., Lewis, J., Raff, M., Roberts, K., and Walter, P. Molecular Biology of The Cell. Garland Science, Taylor and Francis Group, 5th edition, 2008. Cited on p. 10, 12

[64] Li, J., Wang, X., Zhang, T., Wang, C., Huang, Z., Luo, X., and Deng, Y. A review on phospholipids and their main applications in drug delivery systems. Asian Journal of Pharmaceutical Sciences, 10(2):81 - 98, 2015. ISSN 1818-0876. Cited on p. $10,11,12,92$

[65] Bevers, E. M. and Williamson, P. L. Getting to the outer leaflet: Physiology of phosphatidylserine exposure at the plasma membrane. Physiological Reviews, 96 (2):605-645, 2018/07/11 2016. Cited on p. 10, 17, 60, 91

[66] Harayama, T. and Riezman, H. Understanding the diversity of membrane lipid composition. Nature Reviews Molecular Cell Biology, 19(5):281-296, Feb 2018. ISSN 1471-0080. Cited on p. 10, 11, 12, 17

[67] Janmey, P. and Kinnunen, P. Biophysical properties of lipids and dynamic membranes. Trends in Cell Biology, 16(10):538 - 546, 2006. ISSN 0962-8924. Membrane Dynamics. Cited on p. 10, 11

[68] Chiantia, S. and London, E. Lipid bilayer asymmetry. Encyclopedia of Biophysics, pages 1250-1253, 2013. Cited on p. 11

[69] Bangham, A. D. and Horne, R. W. Negative staining of phospholipids and their structural modification by surface-active agents as observed in the electron microscope. Journal of Molecular Biology, 8(5):660-IN10, 1964. Cited on p. 11

[70] Patil, Y. P. and Jadhav, S. Novel methods for liposome preparation. Chemistry and Physics of Lipids, 177:8-18, 2014. Cited on p. 11

[71] Hope, M. J., Bally, M. B., Mayer, L. D., Janoff, A. S., and Cullis, P. R. Generation of multilamellar and unilamellar phospholipid vesicles. Chemistry and Physics of Lipids, 40(2):89-107, 1986. Cited on p. 11 
[72] Johnson, S. M., Bangham, A. D., Hill, M. W., and Korn, E. D. Single bilayer liposomes. Biochimica et Biophysica Acta (BBA) - Biomembranes, 233(3):820826, 1971. Cited on p. 11

[73] Düzgüneş, N. and Nir, S. Mechanisms and kinetics of liposome-cell interactions. Advanced Drug Delivery Reviews, 40(1):3-18, 1999. Cited on p. 11, 60, 67, 91

[74] Seddon, A. M., Curnow, P., and Booth, P. J. Membrane proteins, lipids and detergents: not just a soap opera. Biochimica et Biophysica Acta (BBA) - Biomembranes, 1666(1):105 - 117, 2004. ISSN 0005-2736. Lipid-Protein Interactions. Cited on p. $11,15,61$

[75] Sercombe, L., Veerati, T., Moheimani, F., Wu, S. Y., Sood, A. K., and Hua, S. Advances and challenges of liposome assisted drug delivery. Frontiers in Pharmacology, 6:286, 2015. Cited on p. 11

[76] Allen, T. M. and Cullis, P. R. Liposomal drug delivery systems: From concept to clinical applications. Advanced Drug Delivery Reviews, 65(1):36-48, 2013. Cited on p. 11,92

[77] van Meer, G. and de Kroon, A. I. P. M. Lipid map of the mammalian cell. Journal of Cell Science, 124(1):5, 01 2011. Cited on p. 17

[78] Schindelin, J., Arganda-Carreras, I., Frise, E., Kaynig, V., Longair, M., Pietzsch, T., Preibisch, S., Rueden, C., Saalfeld, S., Schmid, B., and et al. Fiji: an opensource platform for biological-image analysis. Nature Methods, 9(7):676-682, Jul 2012. ISSN 1548-7105. Cited on p. 19, 26, 27, 30, 31, 41, 42, 46, 53, 55, 61

[79] Zheng, W. and Zhao, Q. Establishment and characterization of an immortalized z310 choroidal epithelial cell line from murine choroid plexus. Brain research, 958(2):371-380, 12 2002. Cited on p. 20

[80] Lötvall, J., Hill, A. F., Hochberg, F., Buzás, E. I., Di Vizio, D., Gardiner, C., Gho, Y. S., Kurochkin, I. V., Mathivanan, S., Quesenberry, P., Sahoo, S., Tahara, H., Wauben, M. H., Witwer, K. W., and Théry, C. Minimal experimental requirements for definition of extracellular vesicles and their functions: a position statement from the international society for extracellular vesicles. Journal of Extracellular Vesicles, 3:10.3402/jev.v3.26913, 2014. Cited on p. 21, 22 
[81] Faubel, R. J., , Eichele, G. P. D., Eichele, G. P. D., and Schuh, R. P. D. Spatiotemporal approach to transport dynamics in the mammalian ventricular system, 11 2013. Cited on p. 23, 40

[82] Jaffe, J. D., Miyata, M., and Berg, H. C. Energetics of gliding motility in mycoplasma mobile. J Bacteriol, 186(13):4254-61, Jul 2004. Cited on p. 23, 25

[83] Sbalzarini, I. and Koumoutsakos, P. Feature point tracking and trajectory analysis for video imaging in cell biology. Journal of Structural Biology, 151(2):182 - 195, 2005. ISSN 1047-8477. Cited on p. 26, 43

[84] Chenouard, N., Smal, I., de Chaumont, F., Maška, M., Sbalzarini, I. F., Gong, Y., Cardinale, J., Carthel, C., Coraluppi, S., Winter, M., Cohen, A. R., Godinez, W. J., Rohr, K., Kalaidzidis, Y., Liang, L., Duncan, J., Shen, H., Xu, Y., Magnusson, K. E. G., Jaldén, J., Blau, H. M., Paul-Gilloteaux, P., Roudot, P., Kervrann, C., Waharte, F., Tinevez, J.-Y., Shorte, S. L., Willemse, J., Celler, K., van Wezel, G. P., Dan, H.-W., Tsai, Y.-S., de Solórzano, C. O., Olivo-Marin, J.-C., and Meijering, E. Objective comparison of particle tracking methods. Nature Methods, 11:281 EP -, 01 2014. Cited on p. 26, 43

[85] Revelo, N. H. and Rizzoli, S. O. The membrane marker mcling reveals the molecular composition of trafficking organelles. Curr Protoc Neurosci, 74:2.25.1-21, Jan 2016. Cited on p. 31

[86] Filipe, V., Hawe, A., and Jiskoot, W. Critical evaluation of nanoparticle tracking analysis (nta) by nanosight for the measurement of nanoparticles and protein aggregates. Pharmaceutical Research, 27(5):796-810, May 2010. ISSN 1573-904X. Cited on p. 37,38

[87] Gardiner, C., Ferreira, Y. J., Dragovic, R. A., Redman, C. W., and Sargent, I. L. Extracellular vesicle sizing and enumeration by nanoparticle tracking analysis. Journal of Extracellular Vesicles, 2:10.3402/jev.v2i0.19671, 2013. Cited on p. 37, 38

[88] Maas, S. L., de Vrij, J., van der Vlist, E. J., Geragousian, B., van Bloois, L., Mastrobattista, E., Schiffelers, R. M., Wauben, M. H., Broekman, M. L., and 't Hoen, E. N. N. Possibilities and limitations of current technologies for quantification 
of biological extracellular vesicles and synthetic mimics. Journal of Controlled Release, 200:87 - 96, 2015. ISSN 0168-3659. Cited on p. 38

[89] Bibi, S., Kaur, R., Henriksen-Lacey, M., McNeil, S. E., Wilkhu, J., Lattmann, E., Christensen, D., Mohammed, A. R., and Perrie, Y. Microscopy imaging of liposomes: From coverslips to environmental sem. International Journal of Pharmaceutics, 417(1):138 - 150, 2011. ISSN 0378-5173. Advanced characterization techniques. Cited on p. 38

[90] Freund, J. B., Goetz, J. G., Hill, K. L., and Vermot, J. Fluid flows and forces in development: functions, features and biophysical principles. Development, 139(7): 1229-1245, 2012. ISSN 0950-1991. Cited on p. 44, 56

[91] Dan, G. Allen reference atlas. a digital color brain atlas of the c57bl/6j male mouse - by h. w. dong. Genes, Brain and Behavior, 9(1):128-128, 2018/07/10 2010. Cited on p. 45

[92] Franklin, K. and Paxinos, G. The Mouse Brain in Stereotaxic Coordinates, Compact. Academic Press, 3 edition, March 2008. Cited on p. 48

[93] Bloodgood, R. A. Sensory reception is an attribute of both primary cilia and motile cilia. Journal of Cell Science, 123(4):505, 02 2010. Cited on p. 48

[94] Jain, R., Javidan-Nejad, C., Alexander-Brett, J., Horani, A., Cabellon, M. C., Walter, M. J., and Brody, S. L. Sensory functions of motile cilia and implication for bronchiectasis. Frontiers in bioscience (Scholar edition), 4:1088-1098, 012012. Cited on p. 48

[95] Meste, O., Brau, F., and Guyon, A. Robust estimation of the motile cilia beating frequency. Medical \& Biological Engineering \& Computing, 53(10):1025-1035, 2015. Cited on p. 56

[96] Vieira, O. V., Gaus, K., Verkade, P., Fullekrug, J., Vaz, W. L. C., and Simons, K. Fapp2, cilium formation, and compartmentalization of the apical membrane in polarized madin-darby canine kidney (mdck) cells. Proceedings of the National Academy of Sciences of the United States of America, 103(49):18556-18561, 12 2006. Cited on p. 60 
[97] Rohatgi, R. and Snell, W. J. The ciliary membrane. Current Opinion in Cell Biology, 22(4):541 - 546, 2010. ISSN 0955-0674. Membranes and organelles. Cited on p. 60,77

[98] Lee, K.-D., Hong, K., and Papahadjopoulos, D. Recognition of liposomes by cells: In vitro binding and endocytosis mediated by specific lipid headgroups and surface charge density. Biochimica et Biophysica Acta (BBA) - Biomembranes, 1103(2): 185 - 197, 1992. ISSN 0005-2736. Cited on p. 60, 67

[99] Magarkar, A., Dhawan, V., Kallinteri, P., Viitala, T., Elmowafy, M., Róg, T., and Bunker, A. Cholesterol level affects surface charge of lipid membranes in saline solution. Scientific Reports, 4:5005 EP -, 05 2014. Cited on p. 60, 91

[100] Product Information: ATTO 550. ATTO-TEC GmbH, Am Eichenhang 50, D57076 Siegen, Germany. Cited on p. 60

[101] Product Information: ATTO 647N. ATTO-TEC GmbH, Am Eichenhang 50, D57076 Siegen, Germany. Cited on p. 60

[102] Sandra, A. and Pagano, R. E. Liposome-cell interaction: Studies of lipid transfer using isotopically asymmetric vesicles. The Journal of Biological Chemistry, 254 (7):2244-2249, April 1979. Cited on p. 67

[103] Omran, A. J. A., Saternos, H. C., Althobaiti, Y. S., Wisner, A., Sari, Y., Nauli, S. M., and AbouAlaiwi, W. A. Alcohol consumption impairs the ependymal cilia motility in the brain ventricles. Scientific Reports, 7(1):13652, 2017. Cited on p. 84

[104] Zhou, A., Han, S., and Zhou, Z. J. Molecular and genetic insights into an infantile epileptic encephalopathy -cdkl5 disorder. Frontiers in biology, 12(1):1-6, 022017. Cited on p. 85

[105] Einstein, A. Über die von der molekularkinetischen Theorie der Wärme geforderte Bewegung von in ruhenden Flüssigkeiten suspendierten Teilchen. Annalen der Physik, 322(8):549-560, 2019/08/12 1905. Cited on p. 93 


\section{Acknowledgement}

I would like to thank Prof. Gregor Eichele for giving me the opportunity to work on the cilia mediated transport, for his patience, support and advice.

I would like to thank Prof. Eberhard Bodenschatz and Prof. Christoph Schmidt for being part of my thesis advisory committee and the interesting discussions. I want to thank Prof. Jens Frahm, Dr. Karen Alim and Dr. Thomas Burg for being part of my examination board.

I want to thank Regina Faubel for introducing me to the world of cilia, her motivation, help and friendship. Thank you for all the discussions and marvelous ideas.

I also want to thank Celilia Lo for giving me the opportunity to study cilia liposome interaction in her lab.

I want to thank my colleagues for their support and all the discussions. Especially: Zuzana Ditte, Tamara Rabe, Shoba Kapoor, Christian Westendorf, Dirk Reuter, Annegret Felies, Stefanie Teichmann, Maren Müller, Partho Halder, Ángel Perez Lara and Gurdun Heim.

I want to thank my family Fabian, Sabine, Werner, Jonas, Therese and Ernst for their constant support and love.

And last but not least I would like to thank my friends for their understanding and letting me go into radar silence for weeks and even months without complaining so I could do my $\mathrm{PhD}$. 